HALO GAS AND GALAXY DISK KINEMATICS DERIVED FROM OBSERVATIONS AND ^CDM SIMULATIONS OF Mg II ABSORPTION-SELECTED GALAXIES AT INTERMEDIATE REDSHIFT

This article has been downloaded from IOPscience. Please scroll down to see the full text article.

2010 ApJ 711533

(http://iopscience.iop.org/0004-637X/711/2/533)

The Table of Contents and more related content is available

Download details:

IP Address: 131.215.193.213

The article was downloaded on 12/03/2010 at 21:22

Please note that terms and conditions apply. 


\title{
HALO GAS AND GALAXY DISK KINEMATICS DERIVED FROM OBSERVATIONS AND $\Lambda$ CDM SIMULATIONS OF Mg II ABSORPTION-SELECTED GALAXIES AT INTERMEDIATE REDSHIFT
}

\author{
Glenn G. KacprzaK ${ }^{1,2}$, Christopher W. Churchill ${ }^{2}$, Daniel Ceverino ${ }^{2,3}$, Charles C. Steidel ${ }^{4}$, Anatoly Klypin ${ }^{2}$, \\ AND Michael T. Murphy ${ }^{1}$ \\ ${ }^{1}$ Swinburne University of Technology, Victoria 3122, Australia; gkacprzak@ astro.swin.edu.au, mmurphy@astro.swin.edu.au \\ ${ }^{2}$ New Mexico State University, Las Cruces, NM 88003, USA; cwc@nmsu.edu, ceverino@nmsu.edu, aklypin@nmsu.edu \\ ${ }^{3}$ The Hebrew University, Jerusalem 91904, Israel; ceverino@phys.huji.ac.il \\ ${ }^{4}$ California Institute of Technology, Pasadena, CA 91125, USA; ccs@ astro.caltech.edu \\ Received 2008 July 7; accepted 2009 December 17; published 2010 February 16
}

\begin{abstract}
We obtained ESI/Keck rotation curves of $10 \mathrm{Mg}$ II absorption-selected galaxies $(0.3 \leqslant z \leqslant 1.0)$ for which we have WFPC-2/HST images and high-resolution HIRES/Keck and UVES/VLT quasar spectra of the Mg II absorption profiles. We perform a kinematic comparison of these galaxies and their associated halo $\mathrm{Mg}$ II absorption. For all 10 galaxies, the majority of the absorption velocities lie in the range of the observed galaxy rotation velocities. In $7 / 10$ cases, the absorption velocities reside fully to one side of the galaxy systemic velocity and usually align with one arm of the rotation curve. In all cases, a constant rotating thick-disk model poorly reproduces the full spread of observed Mg II absorption velocities when reasonably realistic parameters are employed. In 2/10 cases, the galaxy kinematics, star formation surface densities, and absorption kinematics have a resemblance to those of high-redshift galaxies showing strong outflows. We find that Mg II absorption velocity spread and optical depth distribution may be dependent on galaxy inclination. To further aid in the spatial-kinematic relationships of the data, we apply quasar absorption-line techniques to a galaxy $\left(v_{c}=180 \mathrm{~km} \mathrm{~s}^{-1}\right)$ embedded in $\Lambda \mathrm{CDM}$ simulations. In the simulations, Mg II absorption selects metal-enriched "halo" gas out to $\sim 100 \mathrm{kpc}$ from the galaxy, tidal streams, filaments, and small satellite galaxies. Within the limitations inherent in the simulations, the majority of the simulated Mg II absorption arises in the filaments and tidal streams and is infalling toward the galaxy with velocities between $-200 \mathrm{~km} \mathrm{~s}^{-1} \leqslant v_{r} \leqslant-180 \mathrm{~km} \mathrm{~s}^{-1}$. The Mg II absorption velocity offset distribution (relative to the simulated galaxy) spans $\sim 200 \mathrm{~km} \mathrm{~s}^{-1}$ with the lowest frequency of detecting $\mathrm{Mg}$ II at the galaxy systematic velocity.
\end{abstract}

Key words: galaxies: halos - galaxies: kinematics and dynamics - intergalactic medium - quasars: absorption lines

Online-only material: color figures

\section{INTRODUCTION}

In a cosmological context, galaxy formation occurs via accretion of gas from the cosmic web and from galaxy-galaxy mergers. The galaxy kinematics reflect these processes. As galaxies evolve, a complex interplay develops between the star formation, which can generate winds, and an array of kinematic structures such as tidal streams, galactic fountains, and filamentary infall that comprise an extended gaseous halo. This overall picture is suggested by observations and $\Lambda \mathrm{CDM}$ cosmological simulations of galaxy formation. However, we lack a thorough understanding, both observationally and theoretically, of how these processes precisely affect the dynamics of galaxies and their extended halos. High-quality, detailed observations are required to further develop this working scenario and produce a comprehensive model of galaxy evolution in the cosmological context.

Observations of local galaxies provide detailed views of gas disks and the inner $\sim 25 \mathrm{kpc}$ of their halos. Oosterloo et al. (2007) obtained deep H I observations of NGC 891 and found lagging, differentially rotating halo gas kinematics with velocities decreasing with distance above the galaxy plane. Lagging halos are observed in several other local galaxies (e.g., Sancisi et al. 2001; Swaters et al. 1997; Rand 2000; Heald et al. 2007). There are also cases where halo gas has been detected with velocities opposite to galaxy rotation (Fraternali et al. 2001; Oosterloo et al. 2007).
Evidence for the accretion of cold gas, which may lower angular momentum and play a role in the development of lagging halos, has also been rapidly accumulating. Several galaxies are observed to have galactic fountains, and be surrounded by $\mathrm{H}$ I cloud complexes, minor merger tidal tails, and intergalactic medium (IGM) filaments (e.g., Heald et al. 2007; Sancisi et al. 2008).

Quasar absorption lines provide powerful probes of halo gas kinematics to large galactocentric distances. In three galaxies, Côté et al. (2005) found that low column density Ly $\alpha$ absorption does not follow galaxy rotation to $D=390 \mathrm{kpc}$. They suggested that the gas arises from the cosmic web. Bowen et al. (2002) found that $\operatorname{Ly} \alpha$ absorption strength correlates with galaxy over density. At large distances, the halo gas kinematics may no longer be coupled to the galaxy kinematics, but reflect the motions of cosmic web.

Quasar absorption-line studies of the $\mathrm{Mg}$ II $\lambda \lambda 2796,2803$ doublet produced by gaseous halos of foreground galaxies (e.g., Bergeron \& Boissé 1991; Le Brun et al. 1993; Steidel et al. 1994; Churchill et al. 1996, 2005; Zibetti et al. 2007; Kacprzak et al. 2007) probe the low ionization metal enriched gas-galaxy dynamics and IGM interface. With Mg II absorption lines, we can study the kinematic conditions of galactic halos over a wide range of redshifts out to projected galactocentric radii of several hundred kpc. Mg II absorption arises in low ionization, metal enriched gas with neutral hydrogen column densities of $10^{16} \mathrm{~cm}^{-2} \lesssim N(\mathrm{HI}) \lesssim 10^{22} \mathrm{~cm}^{-2}$, and thus selects a large 
dynamic range of gas structures in the environments associated with galaxies (Rigby et al. 2002; Churchill et al. 2000).

The idea that $\mathrm{Mg}$ II absorbers could arise from spherical infall, disk-like rotation, or both, is also a topic of much interest. In a small sample of high-resolution $\mathrm{Mg}$ II absorption profiles, Lanzetta \& Bowen (1992) inferred that rotation kinematics dominated at smaller impact parameters, whereas infall kinematics dominated with increasing impact parameter. Armed with a larger high-resolution sample, Charlton \& Churchill (1998) applied statistical tests to a variety of kinematic models and concluded that pure disk rotation and pure halo infall models are ruled out. However, models with contributions from both disk rotation and spherical infall statistically reproduced absorption profiles consistent with observed kinematics.

Among the more extreme structures, are galactic winds generated from star-forming galaxies. At $z \leqslant 1.4$, Tremonti et al. (2007) detected Mg II blueshifted 500-2000 $\mathrm{km} \mathrm{s}^{-1}$ relative to post-starburst host galaxies and Weiner et al. (2009) found $300-1000 \mathrm{~km} \mathrm{~s}^{-1}$ blueshifts in $z \sim 1.4$ star-forming galaxies. In other ionic species, similar outflows have been observed at $z \sim 3$ (Pettini et al. 2001; Shapley et al. 2003; Steidel et al. 2003; Simcoe et al. 2006; Cabanac et al. 2008). None of these surveys have studied the dynamics of the galaxies themselves, which are clearly needed in order to obtain a full picture of the galaxy-halo dynamics.

A direct comparison of the galaxy disk kinematics and absorbing $\mathrm{Mg}$ II halo gas kinematics has been performed for six $z \sim 0.6$ highly inclined galaxies (Steidel et al. 2002; Ellison et al. 2003). Ellison et al. (2003) found that the systemic velocity of a galaxy coincided with the center of the absorption system, which spanned more than $100 \mathrm{~km} \mathrm{~s}^{-1}$ about the systemic velocity. Bond et al. (2001) used expanding shell models to explain that this peculiar absorption profile is likely caused by expanding supernovae-driven superbubbles. Steidel et al. (2002) found that, in four of the five cases, the velocities of all of the absorption components lie to one side of the galaxy systemic redshift. The fifth case had a narrow, weak absorption centered at galaxy systemic velocity. Since the halo gas velocities align in the same sense as the galaxy rotation, the velocity offsets of the absorbing gas relative to the galaxy systemic velocity strongly suggest "disk-like" rotation of the halo gas. Using simple disk halo models, Steidel et al. (2002) concluded that an extension of the disk rotation with a lagging halo component (based upon properties of local galaxies' halo gas kinematics) was able to explain some of the gas kinematics. However, the models were not able to account for the full velocity spreads of the gas.

From a theoretical stand point, semianalytical models and isolated galaxy simulations (e.g., Mo \& Miralda-Escude 1996; Burkert \& Lin 2000; Lin \& Murray 2000; Maller \& Bullock 2004; Chen \& Tinker 2008; Kaufmann et al. 2008; Tinker \& Chen 2008) have been invoked to study isolated galaxy halos. In these models, Mg II absorption arises from condensed, infalling, pressure confined gas clouds within the cooling radius of a hot halo. These models are quite successful at reproducing the general statistical properties of the absorber population. However, they lack the important dynamic influences of the cosmic structure and local environments.

$\Lambda \mathrm{CDM}$ simulations have been able to synthesize the formation and evolution galaxies within large-scale structures. Recently, Ceverino \& Klypin (2009), were able to naturally create, without ad hoc recipes, extended galactic scale outflows and metal enriched multi-phased galactic gas halos. This was accomplished by studying the detailed physics of the formation and evolution of the multi-phase interstellar medium (ISM) in parsec resolution simulations. These same prescriptions were then successfully applied in their large-scale cosmological simulations.

Since these cosmological simulations include all the potential structures that can influence halo gas dynamics and include the local environment, they provide a promising technique for understanding the role of gas in galaxy evolution. The quasar absorption-line method can be applied to simulations to examine structures selected by species such as $\mathrm{Mg}$ II, in the vicinity of galaxies. The goal is to compare directly observed absorbing halo gas kinematics and host galaxy kinematics to those extracted from the simulations. In order to arrive at a deeper understanding, the observations should target redshifts where detailed high-quality kinematics can be obtained for a sample of galaxies with a wide range of orientations with respect to the quasar line of sight.

We have obtained ESI/Keck rotation curves of 10 intermediate redshift $(0.3 \leqslant z \leqslant 1.0)$ galaxies for which we have high-resolution HIRES/Keck or UVES/VLT quasar absorption profiles of $\mathrm{Mg}$ II, as well as WFPC-2/HST images. In this paper, we perform a kinematic comparison of 10 galaxies and their associated halo $\mathrm{Mg}$ II absorption. We define halo gas to be metal enriched structures that give rise to $\mathrm{Mg}$ II absorption such as extraplanar gas, outflows, tidal streams, filaments, and satellite galaxies. We compare our observations with a simple rotating thick disk halo model (similar to the one employed by Steidel et al. 2002) and with the cosmological simulations of Ceverino \& Klypin (2009).

The paper is organized as follows: In Section 2, we present our sample, and explain the data reduction and analysis. In Section 3, we present the results of our galaxy-Mg II absorption kinematic observations, and in Section 4, we compare the observed absorption velocities with a simple disk kinematic halo model. In Section 5, we discuss the details of the cosmological simulations. We study a simulated galaxy and its halo structures in detail. We analyze the integrated total hydrogen and H I column density maps and the absorbing gas velocity distributions. We use these results to infer possible structures and kinematics drivers of that produce the observed $\mathrm{Mg}$ II absorption profiles. We also compute the star formation rate (SFR) and star formation surface density of the simulated galaxy and compare them to previous observational results, and with two of the galaxies in our sample. We end with our conclusions in Section 7. Throughout, we adopt a $h=0.70, \Omega_{\mathrm{M}}=0.3$, and $\Omega_{\Lambda}=0.7$ cosmology.

\section{DATA AND ANALYSIS}

\subsection{Sample Selection}

The selection of the sample presented in the study is based upon three steps. (1) We compiled a list of $\mathrm{Mg}$ II absorbers in high resolution $\left(R=45,000, \mathrm{FWHM} \simeq 6 \mathrm{~km} \mathrm{~s}^{-1}\right.$, with $\mathrm{EW}_{r}(2796) \geqslant 0.02 \AA$ ) HIRES/Keck (Vogt et al. 1994) or UVES/VLT (Dekker et al. 2000) quasar spectra. We make no cut to the sample based upon equivalent width. (2) We then compiled all subsequent deep ground-based imaging and spectroscopic redshift surveys of the quasar fields and selected the galaxies that have confirmed redshifts aligned with $\mathrm{Mg}$ II absorption (Bergeron \& Boissé 1991; Bergeron et al. 1992; Steidel et al. 1994; Lowenthal et al. 1995; Guillemin \& Bergeron 1997; Chen et al. 1998; Lane et al. 1998; this paper). (3) Finally, we selected galaxies for which WFPC-2/HST images 
were available and from which we can extract detailed galaxy morphological parameters (see Kacprzak et al. 2007). The final sample of 10 galaxies have an impact parameter range of $26 \mathrm{kpc} \leqslant D \leqslant 108 \mathrm{kpc}$.

Our goal is to study the relationship between the spatial and kinematics relations between a galaxy and the $\mathrm{Mg}$ II absorbing gas in its vicinity. In our sample, two of the Mg II absorbers appear to be associated with galaxies of similar luminosity that exhibit signs of interaction in the Hubble Space Telescope (HST) images. These galaxies are G1 and G2 in the field of Q0450132, and G1 and G2 in the field of Q1127-145 (see Figures 3(a), (b), and 5, respectively). The Q0450-132 galaxies show tidal asymmetries and have projected separated of $12 \mathrm{kpc}$. There are no additional candidate galaxies in the image within $100 \mathrm{kpc}$ (projected) of the quasar. The Q1127-145 galaxies also exhibit tidal asymmetries. Their projected separation is roughly $50 \mathrm{kpc}$. In the Q1127-145 field, there is a third galaxy, G4, with much smaller luminosity, with a redshift that places it within $70 \mathrm{~km} \mathrm{~s}^{-1}$ of $\mathrm{G} 2$ and $25 \mathrm{~km} \mathrm{~s}^{-1}$ of G1.

These particular systems, which are characterized by two roughly comparable luminosity galaxies with signs of interacting, pose an interesting challenge. They indicate that some $\mathrm{Mg}$ II absorption is arising in the complex environment of a majormajor galaxy interaction. It is probably a fair statement to assert that interacting galaxies of roughly equal luminosity (and that is an important point) will have local environments very different than those of galaxies that clearly have no companion of comparable luminosity. It becomes an intractable problem to discern what portion of the absorption may be arising with gas associated with one or the other galaxy in such a pair. In cases where a single galaxy candidate can be assigned as the luminous host of the $\mathrm{Mg}$ II, it is possible to unambiguously study the spatial and kinematic relationships. Of course, it is always possible a very low luminosity counterpart is below the detection of the images; but such a companion would indicate a minor-major interaction and not a major-major interaction like the Q0450-132 and Q1127-145 pairs. Minor-major interactions would be more akin to the Magellanic galaxies in the $50 \mathrm{kpc}$ vicinity of the Milky Way; they can be considered part of the Milky Way halo. Such a distinction would equally apply to the single galaxies in this sample if there is an unseen minor companion.

In order to keep our sample as uniform as possible for the study of the spatial and kinematic connections between a galaxy and its associated absorbing gas, we limit our analysis to the single galaxy candidates when ambiguities would arise, such as comparison with galaxy inclination, position angle (PA), and impact parameter. There is a very different nature to the major-major interacting pairs toward Q0450-132 and Q1127-145 in that they may have a common gas envelope and therefore provide a slightly different probe of absorption galaxy properties. In cases where ambiguities do not arise in the analysis, we include all the galaxies in our sample.

\subsection{Quasar Spectroscopy}

Details of the HIRES/Keck and UVES/VLT quasar observations are presented in Table 1 . The HIRES spectra (except for Q0836+113) were reduced using IRAF. ${ }^{5}$ The spectrum of

\footnotetext{
5 IRAF is written and supported by the IRAF programming group at the National Optical Astronomy Observatory (NOAO) in Tucson, Arizona. NOAO is operated by the Association of Universities for Research in Astronomy (AURA), Inc. under cooperative agreement with the National Science Foundation.
}

Table 1

Keck + VLT Quasar Observations

\begin{tabular}{lcccc}
\hline \hline QSO Field & $z_{\mathrm{em}}$ & Instrument & Date (UT) & $\begin{array}{c}\text { Exposure } \\
(\mathrm{s})\end{array}$ \\
\hline Q0002+051 & 1.90 & HIRES & 1994 Jul 5 & 2700 \\
Q0229+131 & 2.06 & HIRES & 1999 Feb 8 & 3600 \\
Q0450-132 & 2.25 & HIRES & 1995 Jan 24 & 5400 \\
Q0454-220 & 0.53 & HIRES & 1995 Jan 22 & 5400 \\
Q0836+113 & 2.70 & HIRES & 1998 Feb 26 & 5400 \\
Q1127-145 & 1.18 & UVES & $\ldots{ }^{\mathrm{a}}$ & 24900 \\
Q2206-199 & 2.56 & UVES & $\ldots{ }^{\mathrm{a}}$ & 53503 \\
\hline
\end{tabular}

Notes.

a The Q1127-145 quasar spectrum was obtained over multiple nights. The PIDs for this quasar are 67.A-0567(A) and 69.A-0371(A). The Q2206-199 quasar spectrum was also obtained over multiple nights for the following PIDs 65.O-0158(A), 072.A-0346(A), and 074.A-0201(A).

${ }^{b}$ Data provided by Jason X. Prochaska (Prochaska et al. 2007).

Q0836+113 was reduced using the MAKEE ${ }^{6}$ package. The UVES spectra were reduced using the standard ESO pipeline and a custom code called the UVES Post-Pipeline Echelle Reduction (UVES POPLER; Murphy 2006). The quasar spectra are both vacuum and heliocentric velocity corrected. Analysis of the $\mathrm{Mg}$ II absorption profiles was performed using graphic-based interactive software of our own design (see Churchill et al. 1999, 2000; Churchill \& Vogt 2001) for local continuum fitting, objective feature identification, and measuring absorption properties. The redshift for each Mg II system is computed from the optical depth weighted mean of the absorption profile (see Churchill \& Vogt 2001). The typical absorption redshift uncertainty is $\sim 0.3 \mathrm{~km} \mathrm{~s}^{-1}$. The $\mathrm{Mg}$ II $\lambda 2796$ rest-frame equivalent widths are adopted from Kacprzak et al. (2007). Velocity widths of absorption systems are measured between the pixels where the equivalent width per resolution element recovers to the $1 \sigma$ detection threshold (Churchill et al. 1999).

\subsection{HST Imaging and Galaxy Properties}

All WFPC-2/HST images were reduced using the WFPC-2 Associations Science Products Pipeline (WASPP ${ }^{7}$ ). The WFPC-2 astrometry is calibrated to the USNO2 Catalog (Monet et al. 1998). WASPP data quality verifications include photometric and astrometric accuracy and correctly set zero points. Details of the WFPC-2/HST observations are presented in Table 2. Galaxy apparent and absolute magnitudes are adopted from Kacprzak et al. $(2007,2008)$, respectively. The $m_{F 702 W}$ and $m_{F 814 W}$ magnitudes are based upon the Vega system. As described in Kacprzak et al. (2007), we used GIM2D (Simard et al. 2002) to model the galaxy morphologies, and measured the quasar-galaxy impact parameters, galaxy sky orientations, inclination angles $(i)$, and PAs of their major axes with respect to the quasar line of sight. We fit each galaxy surface brightness profile with a Sérsic bulge component (for $0.2 \leqslant n \leqslant 4.0$ ) and an exponential disk component. Additional modeled galaxy morphological parameters will be presented elsewhere (G. G. Kacprzak et al. 2010, in preparation).

\footnotetext{
6 http://spider.ipac.caltech.edu/staff/tab/makee

7 Developed by the Canadian Astronomy Data Centre (CADC) and the Space Telescope-European Coordinating Facility (ST-ECF):

http://archive.stsci.edu/hst/wfpc2/pipeline.html.
} 
Table 2

WFPC-2/HST Observations

\begin{tabular}{llcc}
\hline \hline QSO Field & Filter & $\begin{array}{c}\text { Exposure } \\
(\mathrm{s})\end{array}$ & PID/PI \\
\hline Q0002+051 & $F 702 W$ & 4600 & $5984 /$ Steidel \\
Q0229+131 & $F 702 W$ & 5000 & $6557 /$ Steidel \\
Q0450-132 & $F 702 W$ & 2500 & $5984 /$ Steidel \\
Q0454-220 & $F 702 W$ & 1200 & $5098 /$ Burbidge \\
Q0836+113 & $F 702 W$ & 5000 & $6557 /$ Steidel \\
Q1127-145 & $F 814 W$ & 4400 & $9173 /$ Bechtold \\
Q2206-199 & $F 702 W$ & 5000 & $6557 /$ Steidel \\
\hline
\end{tabular}

Table 3

Keck-II/ESI Observations

\begin{tabular}{ccccc}
\hline \hline QSO Field & $z_{\text {abs }}$ & Date (UT) & $\begin{array}{c}\text { Exposure } \\
(\mathrm{s})\end{array}$ & $\begin{array}{c}\text { Slit } \\
\text { PA }\end{array}$ \\
\hline Q0002+051 G1 & 0.851407 & 2001 Oct 16 & 7200 & -8.5 \\
Q0229+131 G1 & 0.417337 & 2006 Dec 24 & 6500 & 134 \\
Q0450-132 G1 & 0.493937 & 2006 Dec 24 & 5300 & -13 \\
Q0450-132 G2 & 0.493937 & 2006 Dec 24 & 5300 & -13 \\
Q0454-220 G1 & 0.483338 & 2006 Dec 24 & 4800 & 276 \\
Q0836+113 G1 & 0.786726 & 2006 Dec 24 & 5300 & 130 \\
Q0836+113 G2 & $\ldots . . \mathrm{a}$ & 2006 Dec 24 & 5300 & 130 \\
Q1127-145 G1 & 0.312710 & 2006 Dec 24 & 3900 & 129 \\
Q1127-145 G2 & 0.312710 & 2006 Dec 24 & 4200 & 87.5 \\
Q1127-145 G3 & 0.328266 & 2006 Dec 24 & 600 & 87.5 \\
Q2206-199 G1 & 1.017040 & 2001 Oct 16 & 1800 & 75 \\
\hline
\end{tabular}

Note. ${ }^{\text {a }}$ There is no Mg II absorption associated with Q0836+113 G2 in the literature. Our HIRES data do not provide the necessary wavelength coverage.

\subsection{Galaxy Spectroscopy}

The ESI/Keck (Sheinis et al. 2002) galaxy spectra were obtained over two nights; two were obtained in 2001 October and eight were obtained in 2006 December. Details of the ESI/ Keck observations are presented in Table 3. For each galaxy, the slit PA was chosen to lie along the galaxy major axis (except for Q450-132 G2). The slit length is $20^{\prime \prime}$. Thus in some cases, we were able to simultaneously position two galaxies on a slit. Exposure times range between 600 and 7200 s per galaxy. The wavelength coverage of ESI is $4000-10000 \AA$, which allows us to obtain multiple emission lines (such as [O II] doublet, $\mathrm{H} \beta$, [O III] doublet, $\mathrm{H} \alpha,[\mathrm{N}$ II] doublet, etc.) with a velocity resolution of $11 \mathrm{~km} \mathrm{~s}^{-1}$ pixel $^{-1}\left(\mathrm{FWHM} \sim 45 \mathrm{~km} \mathrm{~s}^{-1}\right)$.

In 2001 October, the data were obtained with a $1^{\prime \prime}$ slit and $2 \times 2$ binning. The mean seeing was 0.'7 (FWHM) with partial cloud coverage. In 2006 December, the data were obtained using a 0 '.75 slit with $2 \times 1$ binning. Binning by two in the spatial directions results in pixel sizes of 0 '.27-0.'34 over the orders of interest. The mean seeing was 0'.8 (FWHM) with clear skies.

The data were reduced using the standard echelle package in IRAF. We used internal quartz illumination flat fields to eliminate pixel to pixel variations. In the science frames, sky subtraction was performed by fitting a polynomial function to each spatial column. A quasar or bright star spectrum in the same field was obtained and used as a trace in order to facilitate the extraction of the galaxy spectrum. A spatially integrated spectrum was extracted in order to obtain an accurate galaxy redshift from the centroids of multiple emission lines. The lines listed in the legends of Figures 2-6 were used to determine the galaxy redshifts. The rest-frame vacuum wavelength used for each emission line was obtained from the National Institute
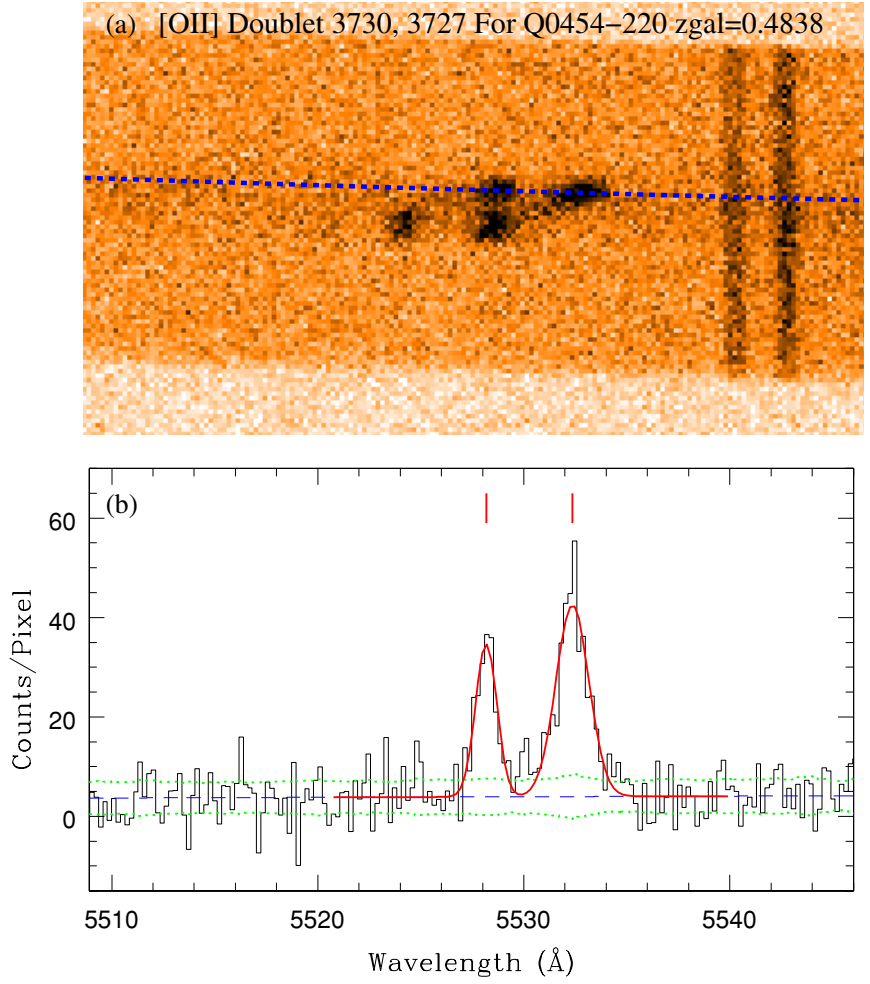

Figure 1. (a) Two-dimensional spectral region around the [O II] doublet from the $z=0.4838$ galaxy in the quasar field Q0454-220. The [O II] is spatially resolved and extends roughly $5^{\prime \prime}$ on the sky. The two sky lines extend the length of the $20^{\prime \prime}$ slit. (b) A one-dimensional extraction of the above twodimensional image (thick dashed line in top panel) summed over 3 pixels in the spatial direction. The galaxy continuum fit is indicated by the dashed line. The $1 \sigma$ uncertainty in the continuum is shown by the dotted lines bracketing the continuum. The sky lines and sky signal have been subtracted out. The solid line shows the Gaussian fit to the emission lines and the tick marks indicate the centroids of the line.

(A color version of this figure is available in the online journal.)

of Standards and Technology (NIST) database. Each spectrum was wavelength calibrated using CuArXe arc line lamps. Spectra were calibrated in IRAF using standard stars taken during the night of the observation. The flux is accurate to $\sim 10 \%$ and we have made no corrections for slit loss, or Galactic reddening.

In Figure 1, we show an example of a two-dimensional spectrum of the spatially resolved [O II] doublet from the $z=0.4838$ galaxy in the Q0454-220 field. We used a Gaussian fitting algorithm (see Churchill et al. 2000), which computes best-fit Gaussian amplitudes, widths, and centers (redshift), to the galaxy emission and absorption lines. The galaxy redshift was computed from the mean redshift of all the detected lines. Emission lines and absorption lines used to calculate the galaxy redshift must have been detected at the $3 \sigma$ level. The adopted redshift uncertainty for each galaxy was computed from the standard deviation in the redshifts computed from each emission line. The galaxy redshifts are listed in Table 4; their accuracy ranges from 2 to $45 \mathrm{~km} \mathrm{~s}^{-1}$. The galaxy velocity offsets from the optical depth weighted mean Mg II absorption are also listed in Table 4 and range from -12 to $+168 \mathrm{~km} \mathrm{~s}^{-1}$.

The rotation curve extraction was performed following the methods of Vogt et al. (1996) and Steidel et al. (2002). We extract individual one-dimensional spectra by summing 3-pixel wide apertures (corresponding to approximately one resolution element of $0{ }^{\prime \prime} 82-1{ }^{\prime \prime} 01$ ) at 1 pixel spatial increments along the slit. An error spectrum is also extracted for each of these apertures. 
Table 4

Mg II Absorption and Galaxy Redshifts

\begin{tabular}{ccllc}
\hline \hline QSO Field & $z_{\text {abs }}$ & $z_{\text {gal }}$ & \multicolumn{1}{c}{$\sigma z_{\text {gal }}$} & $\begin{array}{c}\Delta v_{r}{ }^{\mathrm{a}} \\
\left(\mathrm{km} \mathrm{s}^{-1}\right)\end{array}$ \\
\hline Q0002+051 G1 & 0.851407 & 0.85180 & 0.000066 & -66 \\
Q0229+131 G1 & 0.417337 & 0.4167 & 0.00020 & +135 \\
Q0450-132 G1 & 0.493937 & 0.4941 & 0.00015 & -33 \\
Q0450-132 G2 & 0.493937 & 0.4931 & 0.00012 & +168 \\
Q0454-220 G1 & 0.483338 & 0.48382 & 0.000066 & -98 \\
Q0836+113 G1 & 0.786726 & 0.78682 & 0.000028 & -16 \\
Q1127-145 G1 & 0.312710 & 0.3132 & 0.00020 & -112 \\
Q1127-145 G2 & 0.312710 & 0.3124 & 0.00013 & +71 \\
Q1127-145 G3 & 0.328266 & 0.32847 & 0.000027 & -46 \\
Q2206-199 G1 & 1.017040 & 1.01655 & 0.000013 & +73 \\
\hline
\end{tabular}

Note. ${ }^{\mathrm{a}} \Delta v_{r}$ is the rest-frame velocity offset between the mean $\mathrm{Mg}$ II $\lambda 2976$ absorption line and the galaxy where, $\Delta v_{r}=c\left(z_{\mathrm{abs}}-z_{\mathrm{gal}}\right) /\left(1+z_{\mathrm{gal}}\right) \mathrm{km} \mathrm{s}^{-1}$.

To obtain accurate wavelength calibrations, we extracted spectra of $\mathrm{CuArXe}$ arc line lamps at the same spatial pixels as the extracted galaxy spectra. Fitted arc lamp exposures (CuArXe) provided a dispersion solution accurate to $\sim 0.15 \AA$, or about $6.5 \mathrm{~km} \mathrm{~s}^{-1}$ at the wavelengths of interest. Galaxy spectra are both vacuum and heliocentric velocity corrected for comparison with the absorption-line kinematics. Each galaxy emission line (or absorption line in some cases) was fitted with a single Gaussian (except the [O II] doublet was fit with a double Gaussian) in order to extract the wavelength centroid for each emission line.

An example of a 3-pixel wide spectral extraction from the two-dimensional spectrum is shown in Figure 1(b) where the [O II] $\lambda 3727$ and $\lambda 3730$ lines are detected at the $22 \sigma$ and $17 \sigma$ level, respectively (the significance level is the ratio of the measured equivalent width to the uncertainty in the equivalent width based upon error propagation using the error spectrum extracted for the same 3-pixel aperture). The dashed line in Figure 1(a) provides the spatial cut for which the spectrum in Figure 1(b) is illustrated. The velocity offsets for each emission line in each extraction were computed with respect to the redshift zero point determined for the galaxy (Table 4). The rotation curves for the 10 galaxies obtained with ESI/Keck are presented in Figures 2-6.

\section{DISCUSSION OF INDIVIDUAL FIELDS}

Here, we discuss the halo gas and galaxy kinematics of 10 galaxies in seven different quasar fields. In Table 5, we list all the galaxies in each field that have spectroscopically confirmed redshifts. The table columns are (1) the quasar field, (2) the galaxy ID, (3) the galaxy redshift, (4) the reference(s) for the galaxy identification, (5) the quasar-galaxy impact parameter, $D$, and uncertainty, (6) the $\mathrm{Mg}$ II absorption redshift, and (7) the rest-frame $\mathrm{Mg}$ II $\lambda 2796$ equivalent width, $W_{r}(2796)$, and uncertainty. Three new galaxies (Q0002+051 G1, Q0836+133 G2, and Q1127-145 G3) have been spectroscopically identified in this work.

Most of the quasar fields listed in Table 5 have been spectroscopically surveyed for all galaxies with $m_{r} \lesssim 23$, which translates to $L_{\star} \simeq 0.08 L_{\star}$ for $z \simeq 0.5$, out to $\sim 1^{\prime}$ (e.g., Steidel et al. 1994; Guillemin \& Bergeron 1997; Chen et al. 1998). These works have been instrumental in developing our current picture of the galaxy-absorber connection at intermediate redshifts. It remains possible that additional galaxies below this luminosity lurk in front of the quasar and may be associated with the absorbing gas. It is also possible that a less than $100 \%$ completeness in the confirmation of galaxy redshifts may result in an additional galaxy or galaxies also associated with the

Table 5

Mg II Absorption and Galaxy Redshift Field Survey

\begin{tabular}{|c|c|c|c|c|c|c|c|}
\hline QSO Field & $\begin{array}{c}\text { Galaxy } \\
\text { ID }\end{array}$ & $z_{\text {gal }}$ & $\begin{array}{c}z_{\text {gal }} \\
\text { Reference }^{a}\end{array}$ & $D(\mathrm{kpc})$ & $z_{\mathrm{abs}}$ & $W_{r}(2796) \AA$ & $\begin{array}{l}\text { Galaxies in } \\
\text { This Study }\end{array}$ \\
\hline \multirow[t]{3}{*}{$\mathrm{Q} 0002+051$} & G1 & 0.85180 & 1 & $25.9 \pm 0.5$ & 0.851407 & $1.119 \pm 0.013$ & $\mathrm{X}$ \\
\hline & $\mathrm{G} 2$ & 0.592 & 2 & $36.2 \pm 0.4$ & 0.591365 & $0.102 \pm 0.002$ & \\
\hline & G3 & 0.298 & 2 & $59.3 \pm 0.3$ & 0.298059 & $0.246 \pm 0.004$ & \\
\hline Q0229+131 & G1 & 0.4167 & 1,3 & $37.5 \pm 0.5$ & 0.417337 & $0.816 \pm 0.022$ & $X$ \\
\hline \multirow[t]{2}{*}{ Q0450-132 } & G1 & 0.4941 & 1,2 & $50.1 \pm 0.4$ & 0.493937 & $0.674 \pm 0.026$ & $\mathrm{X}$ \\
\hline & $\mathrm{G} 2$ & 0.4931 & 1,2 & $62.7 \pm 0.7$ & 0.493937 & $0.674 \pm 0.026$ & $\mathrm{X}$ \\
\hline Q0454-220 & G1 & 0.48382 & 1,4 & $107.9 \pm 0.8$ & 0.483338 & $0.426 \pm 0.007$ & $X$ \\
\hline Q0454-220 & G2 & 0.3818 & 4 & $103.4 \pm 0.3$ & $\cdots$ & $<0.02(3 \sigma)$ & \\
\hline \multirow[t]{2}{*}{ Q0836+113 } & G1 & 0.78682 & 1,5 & $26.9 \pm 0.9$ & 0.786726 & $2.148 \pm 0.023$ & $\mathrm{X}$ \\
\hline & $\mathrm{G} 2$ & 0.48288 & 1 & $29.1 \pm 0.3$ & $\ldots$ & & \\
\hline \multirow[t]{4}{*}{ Q1127-145 } & G1 & 0.3132 & 1,3 & $45.6 \pm 0.3$ & 0.312710 & $1.773 \pm 0.006$ & $\mathrm{X}$ \\
\hline & $\mathrm{G} 2$ & 0.3124 & 1,3 & $81.0 \pm 0.3$ & 0.312710 & $1.773 \pm 0.006$ & $\mathrm{X}$ \\
\hline & G3 & 0.32847 & 1 & $91.4 \pm 0.2$ & 0.328266 & $0.029 \pm 0.003$ & $\mathrm{X}$ \\
\hline & G4 & 0.3121 & 6 & $18.2 \pm 0.3$ & 0.312710 & $1.773 \pm 0.006$ & \\
\hline \multirow[t]{3}{*}{ Q2206-199 } & G1 & 1.01655 & 1,7 & $104.6 \pm 1.4$ & 1.017040 & $1.057 \pm 0.005$ & $\mathrm{X}$ \\
\hline & $\mathrm{G} 2$ & 0.948 & 7 & $87.2 \pm 0.5$ & 0.948361 & $0.253 \pm 0.002$ & \\
\hline & $\mathrm{G} 3^{\mathrm{c}}$ & 0.755 & 8 & $44.2 \pm 0.7$ & 0.751923 & $0.886 \pm 0.003$ & \\
\hline
\end{tabular}

Notes.

a Galaxy identification: (1) This paper; (2) Steidel et al. 1994; (3) Bergeron \& Boissé 1991; (4) Chen et al. 1998; (5) Lowenthal et al. 1995; (6) Lane et al. 1998; (7) Bergeron et al. 1992; and (8) Guillemin \& Bergeron 1997. We list the redshift for galaxies that were derived for this work.

b There is no Mg II absorption associated with Q0836+113 G2 in the literature. Our HIRES data do not provide the necessary wavelength coverage.

${ }^{c}$ G3 was reported as a galaxy by Guillemin \& Bergeron (1997). However, our spectroscopic observations reveal that this unresolved object is a Galactic star. 
(b)
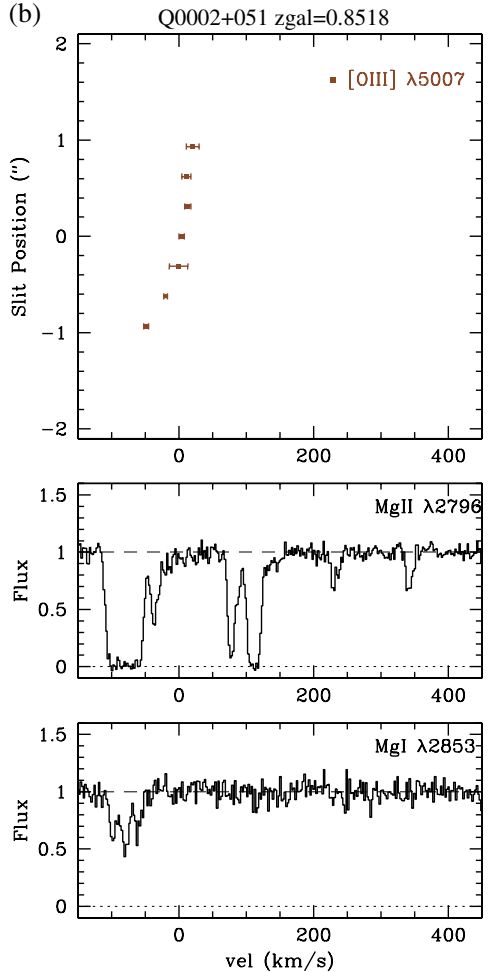

Q0002+051 zgal=0.8518

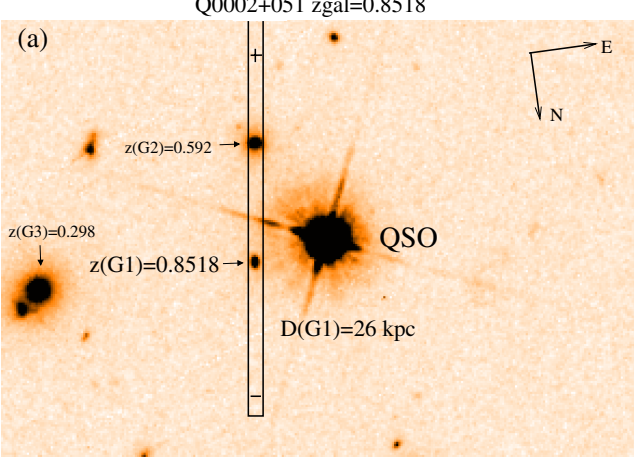

Q0229+131 zgal $=0.4167$

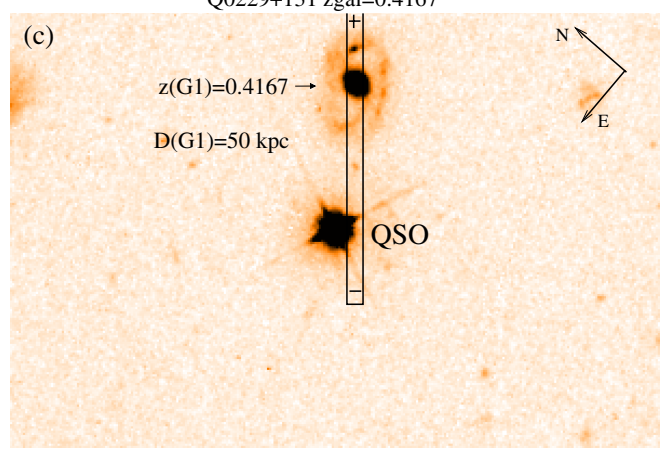

(d)
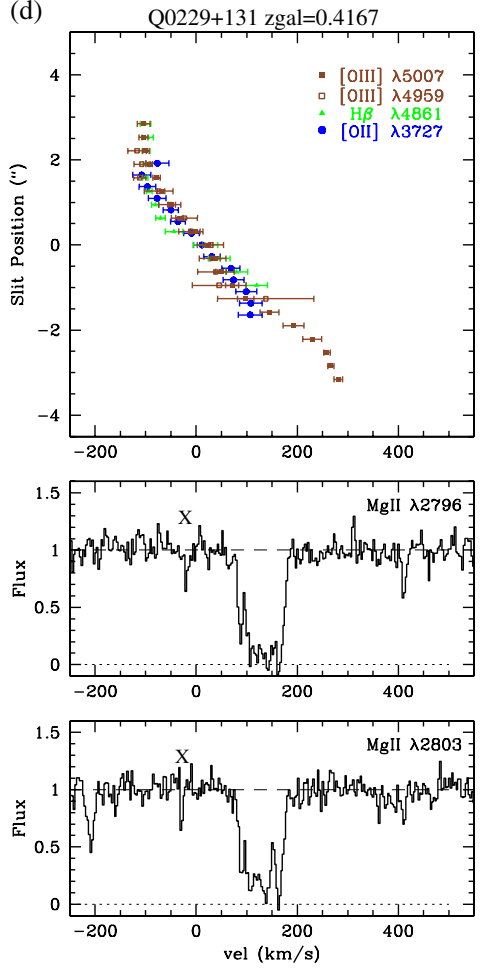

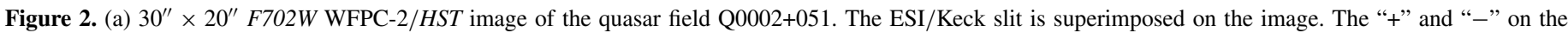

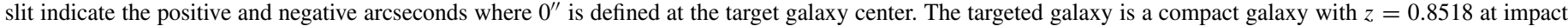

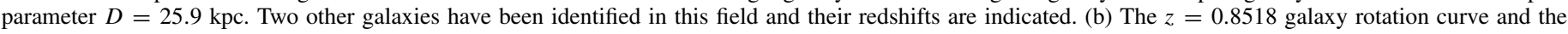

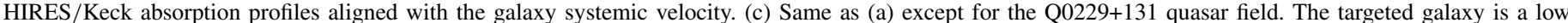

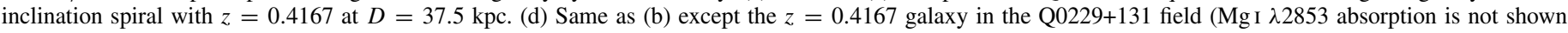

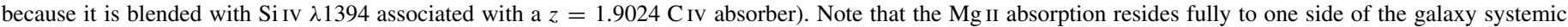
velocity and also aligns with one arm of the rotation curve.

(A color version of this figure is available in the online journal.)

absorption. The concerns and caveats associated with incorrect identifications and the conclusion drawn for those works therefore also apply to this study. Galaxies fainter than $m_{r} \sim 23$, or low-mass galaxies hidden by the quasar point-spread function (PSF), such as satellites or minor companions, can be considered part of the halo of the large host galaxy.

In the following subsections, we discuss only the 10 galaxies selected for this study (see Column 8 of Table 5). Detailed images of the galaxies are presented in Kacprzak et al. (2007). A summary of the impact parameters, galaxy maximum rotation velocities, and GIM2D model inclinations and PAs (PA $\equiv$ angle between the galaxy major axis and the quasar line of sight) is listed in Table 6. Galaxy redshifts will only be quoted to four significant figures from here on for simplicity. We will later discuss kinematic halo models in Section 4.

\section{1. $Q 0002+051 G 1$}

A WFPC-2/HST image of the Q0002+051 field is shown is Figure 2(a). The $z=0.8518$ galaxy, G1, was targeted for this study. It was first assumed by Steidel et al. (1994) to be the absorbing galaxy associated with the $\mathrm{Mg}$ II absorption at $z=0.851407$ given its colors and its proximity to the quasar. We report the first redshift confirmation of this galaxy. The galaxy redshift was identified by an [O III] and a weak [O II] emission line (the [O II] line was used only for the determination of the galaxy redshift and provided no kinematic information). In Figure 2(a), we note that the ESI/Keck slit was placed across both G1 and G2 (where "+" and "-" on the slit indicate the positive and negative arcseconds relative to the center of the galaxy, respectively). We did not detect any identifiable emission lines from the galaxy G2.

The Mg II absorption was first reported by Bechtold et al. (1984) and then confirmed by Steidel \& Sargent (1992). The HIRES/Keck spectrum was originally presented by Churchill et al. (1996) and has $W_{r}(2796)=1.119 \AA$.

The G1 galaxy has a compact morphology. It has an absolute $B$-band magnitude of $M_{B}=-21.3$ which translates to a luminosity $L_{B}=0.92 L_{B}^{*}$. The galaxy has inclination angle $i=38^{\circ}$ and $\mathrm{PA}=43^{\circ} . \mathrm{G} 1$ is at a projected distance of $D=25.9 \mathrm{kpc}$ from the quasar line of sight. In Figure 2(b), the spatial radial velocity, as derived from [O III], is shown. It appears G1 is not predominantly rotating. The projected velocity shear is $49 \mathrm{~km} \mathrm{~s}^{-1}$. Displayed in the lower panels are the $\mathrm{Mg}$ II and $\mathrm{Mg}$ I absorption profiles, shown on the same velocity scale. The mean optical depth absorption redshift is offset by $-66 \mathrm{~km} \mathrm{~s}^{-1}$ from the galaxy systemic velocity. This Mg II absorption system is the most kinematically complex absorption profile in our sample. Four absorption subsystems are spread out over a total velocity of $\sim 475 \mathrm{~km} \mathrm{~s}^{-1}$. The blueshifted extreme wing of G1's velocity shear is partially consistent with the velocities of the dominant saturated component of the $\mathrm{Mg}$ II. However, it is inconsistent with the $\mathrm{Mg}$ II gas at large positive velocities. The MgI gas, which may trace more neutral and higher column density gas, has velocities is also inconsistent with the velocities of G1. 
(b) Q0450-132 zgal: G1 $=0.4941, \mathrm{G} 2=0.4931$
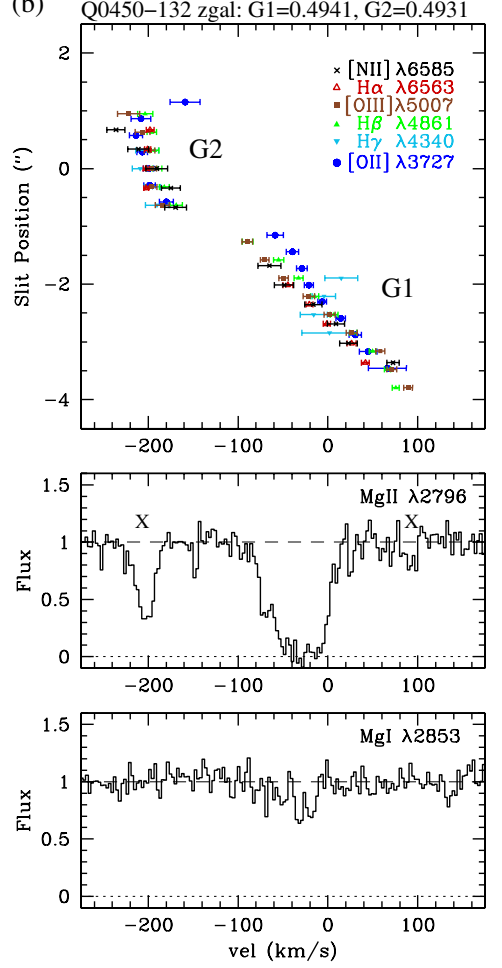

(a)

Q0450-132 zgal: $\mathrm{G} 1=0.4942, \mathrm{G} 2=0.493$

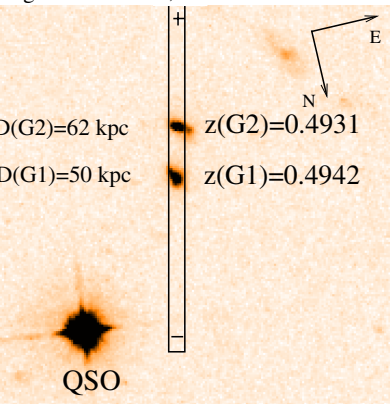

Q0454-220 zgal=0.4838

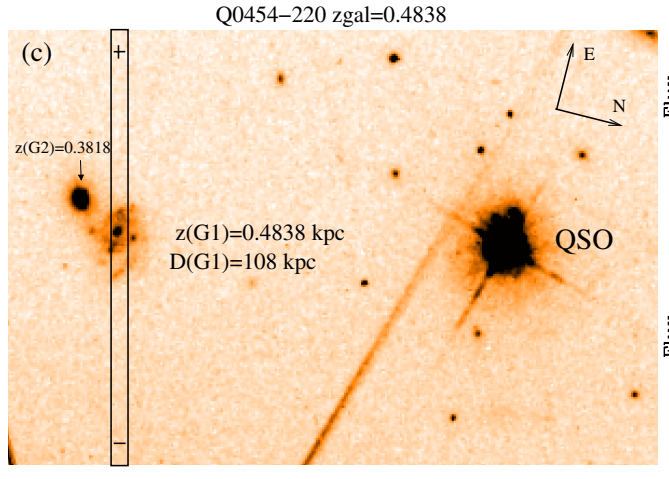

(d)
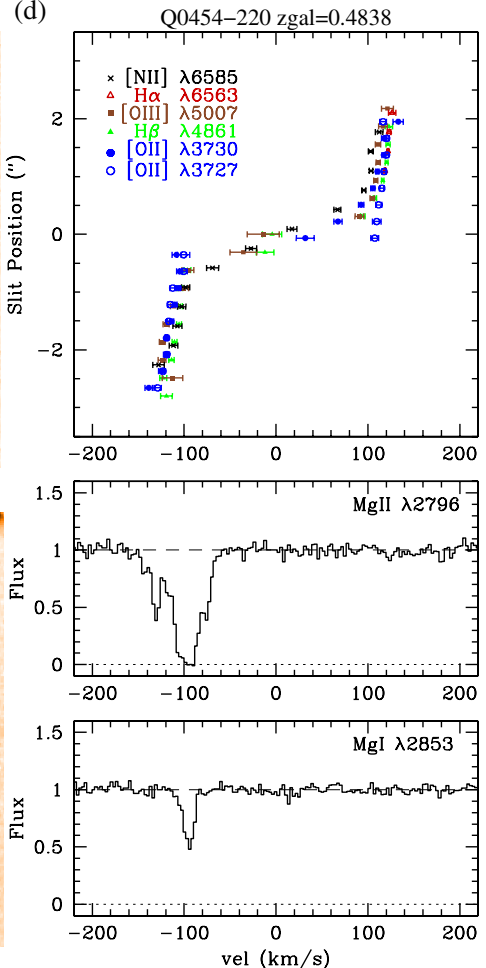

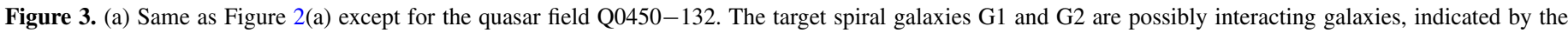

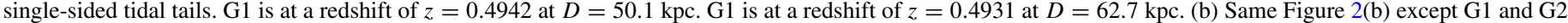

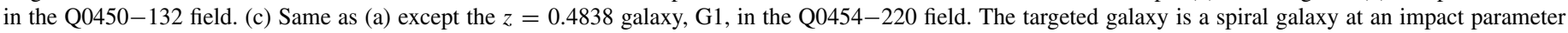
$D=107.9 \mathrm{kpc}$. (d) Same as (b) except for the G1 galaxy in the Q0454-220 field.

(A color version of this figure is available in the online journal.)

\section{2. $Q 0229+131 G 1$}

A WFPC-2/HST image of the Q0229+131 field is shown in Figure 2(c). The $z=0.4167$ galaxy, G1, was initially spectroscopically identified by Bergeron \& Boissé (1991). The first detection of $\mathrm{Mg}$ II absorption at the galaxy redshift was reported by Sargent et al. (1988). A HIRES/Keck spectrum of this absorber was first presented by Churchill et al. (2005) and has $W_{r}(2796)=0.816 \AA$.

This moderately inclined, $i=58^{\circ}$, bulge-dominated galaxy has two large grand design spiral arms similar to a local $\mathrm{Sb}$ galaxy. One of the arms contains a bright $\mathrm{H}_{\text {II }}$ region. The galaxy position angle is $\mathrm{PA}=22^{\circ}$. G1 has an absolute $B$-band magnitude of $M_{B}=-20.8$ which translates to $L_{B}=0.74 L_{B}^{*}$. G1 is at a projected distance of $D=37.5 \mathrm{kpc}$ from the quasar line of sight.

In Figure 2(d), the rotation curve, derived from [O II], $\mathrm{H} \beta$, and [O III] emission lines, is shown. The asymmetric rotation curve has a maximum observed velocity of $280 \mathrm{~km} \mathrm{~s}^{-1}$. The asymmetry may be a result of the asymmetric spiral arms. Below the rotation curve, the $\mathrm{Mg}$ II $\lambda \lambda 2796,2803$ absorption profiles are shown on the same velocity scale. The mean absorption redshift is offset by $+135 \mathrm{~km} \mathrm{~s}^{-1}$ from the galaxy systemic velocity. The $\mathrm{Mg}$ I profile is not shown here since it is completely bended with a four component Si IV $\lambda 1394$ complex from a $z=1.9024$ C IV absorber (Sargent et al. 1988). This Mg II absorption system has a velocity spread of roughly $112 \mathrm{~km} \mathrm{~s}^{-1}$ with a single cloud residing roughly $+280 \mathrm{~km} \mathrm{~s}^{-1}$ from the main component. The main absorption component aligns with the redshifted wing of the galaxy rotation curve arising from the spiral arm nearest the quasar. The galaxy rotation velocities are not consistent with the outlying cloud.

\section{3. $Q 0450-132 G 1, G 2$}

A WFPC-2/HST image of the Q0450-132 field is shown in Figure 3(a). Two galaxies were placed along the same slit. The redshifts of this double pair were initially obtained by Steidel et al. (1994). This absorption system was discovered during the survey of Sargent et al. (1988) and the HIRES/Keck spectrum was first presented by Churchill (1997). The Mg II absorption has an equivalent width of $W_{r}(2796)=0.674 \AA$.

The galaxy closer to the quasar, G1, has a redshift of $z=0.4941$ and is at an impact parameter of $D=50.1 \mathrm{kpc}$. The galaxy has an inclination of $i=66^{\circ}$ with a PA $=42^{\circ}$. G1 has an absolute magnitude of $M_{B}=-19.7$ which translates to a $L_{B}=0.25 L_{B}^{*}$ galaxy. The galaxy further from the quasar, $\mathrm{G} 2$, has a redshift of $z=0.4931$ and has an impact parameter of $62.7 \mathrm{kpc}$. The galaxy has an inclination of $i=75^{\circ}$ with a $\mathrm{PA}=54^{\circ} . \mathrm{G} 2$ has an absolute magnitude of $M_{B}=-19.7$ which translates to $L_{B}=0.26 L_{B}^{*}$. The galaxies are spatially separated by only $14 \mathrm{kpc}$ projected, and by a line-of-sight velocity of $201 \mathrm{~km} \mathrm{~s}^{-1}$. Both galaxies appear to have single-sided tidal tails and show strong morphological evidence of a previous interaction or harassment.

The rotation curves of both galaxies, as derived from [O II], $\mathrm{H} \gamma, \mathrm{H} \beta,[\mathrm{O} \mathrm{III}], \mathrm{H} \alpha$, and [N $\mathrm{II}]$, are presented in Figure 3(b). The maximum observed rotation velocities for G1 and G2 are $98 \mathrm{~km} \mathrm{~s}^{-1}$ and $47 \mathrm{~km} \mathrm{~s}^{-1}$, respectively. Below the rotation curve, the $\mathrm{Mg}$ II and $\mathrm{Mg}$ I absorption profiles are shown on the 
(b)
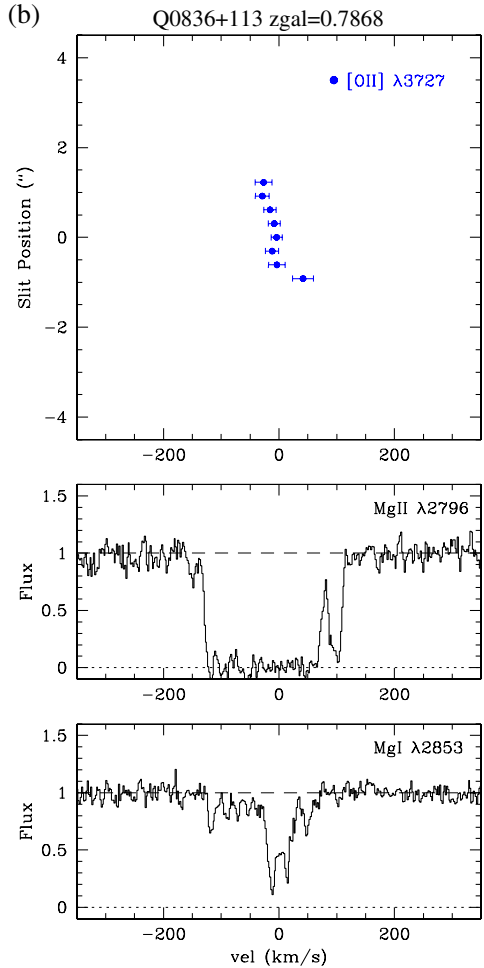

$\mathrm{Q} 0836+113 \mathrm{zgal}=0.7868$

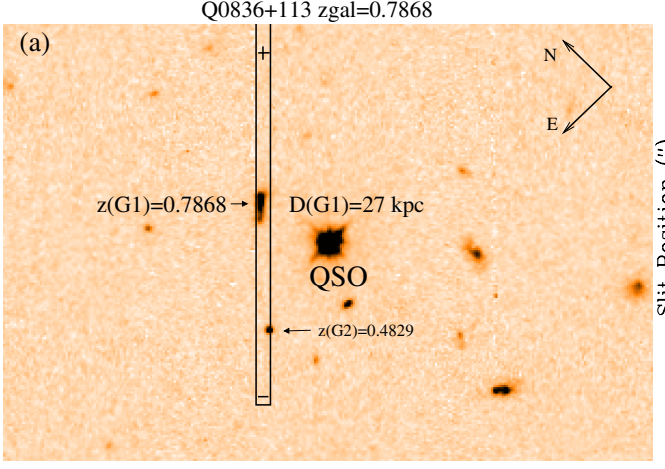

Q1127-145G3 zgal=0.3285

(c)

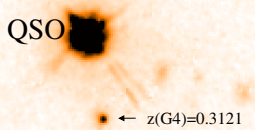

(d)
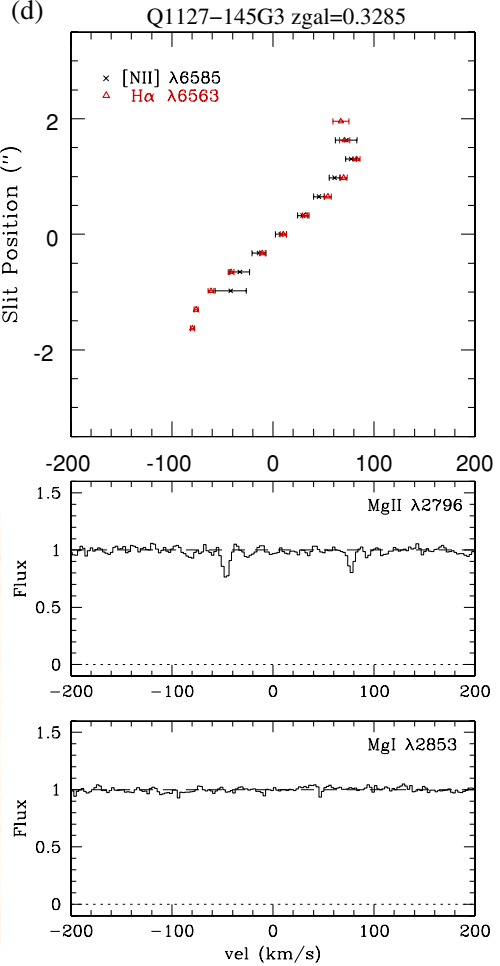

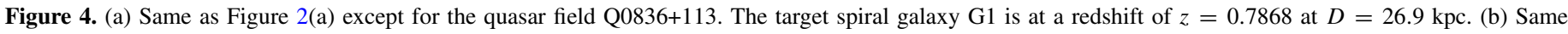

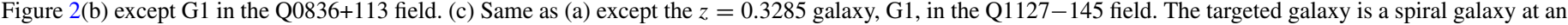
impact parameter $D=91.4 \mathrm{kpc}$. (d) Same as (b) except for the G1 galaxy in the Q1127-145 field.

(A color version of this figure is available in the online journal.)

same velocity scale. The mean absorption redshift is offset by $-33 \mathrm{~km} \mathrm{~s}^{-1}$ from the galaxy systemic velocity of G1. The Mg II absorption is a single kinematic region with a velocity width of $103 \mathrm{~km} \mathrm{~s}^{-1}$. The absorption aligns, in velocity, with the blueshifted wing of the rotation curve of G1. The Mg I aligns more closely to the systemic velocity of G1. The galaxy G2 does not have much rotation since the slit PA is close to its minor axis.

\section{4. $Q 0454-220 G 1$}

A WFPC-2/HST image of the Q0454-220 field is shown in Figure 3(c). The $z=0.4838$ galaxy, G1, was targeted for this study. G1 was spectroscopically identified by Chen et al. (1998). The Mg II absorption was first reported by Bergeron \& Kunth (1984) and then confirmed by Tytler et al. (1987). The HIRES/ Keck spectrum was originally presented by Churchill \& Vogt (2001). The $\mathrm{Mg}$ II equivalent width is $W_{r}(2796)=0.426 \AA$.

G1 is a spiral galaxy that has a perturbed morphology with one extended spiral arm. It has a compact bulge and several bright $\mathrm{H}$ II regions. The galaxy has an inclination of $i=41^{\circ}$ and a position angle of $\mathrm{PA}=76^{\circ}$. It has an absolute $B$-band magnitude of $M_{B}=-21.0$ which translates to $L_{B}=0.90 L_{B}^{*}$. G1 is at a projected distance of $D=107.9 \mathrm{kpc}$ from the quasar line of sight.

The rotation curve, as derived from [O II], $\mathrm{H} \beta$, [O III], $\mathrm{H} \alpha$, and [N $\mathrm{II}]$, is presented in Figure 3(d). The rotation curve flattens out at a maximum observed velocity of $138 \mathrm{~km} \mathrm{~s}^{-1}$. Below the rotation curve, the $\mathrm{Mg}$ II and the $\mathrm{Mg}_{\mathrm{I}}$ absorption profiles are shown on the same velocity scale. The mean absorption redshift is offset by $-98 \mathrm{~km} \mathrm{~s}^{-1}$ from the galaxy systemic velocity. This $\mathrm{Mg}$ II absorption is a single kinematic system having a velocity spread of roughly $93 \mathrm{~km} \mathrm{~s}^{-1}$. The blueshifted component of the rotation curve has velocities that are consistent with the $\mathrm{Mg}$ II and $\mathrm{Mg}$ I absorption profiles.

\section{5. $Q 0836+113 G 1$}

A WFPC-2/HST image of the Q0836+113 field is shown in Figure 4(a). The $z=0.7868$ galaxy, G1, was initially spectroscopically identified by Lowenthal et al. (1995). The first $\mathrm{Mg}$ II absorption detection at the galaxy redshift was reported by Turnshek et al. (1989). The HIRES/Keck absorption profiles of this absorber are first presented in this work (Figure 4(b)) and has $W_{r}(2796)=2.15 \pm 0.02 \AA$.

The galaxy G1 appears to be an edge-on spiral with an asymmetric brightness profile. The morphology is similar to that of the two galaxies in Q0450-132, which have singlesided tidal tails. This almost edge-on galaxy has an inclination of $i=78^{\circ}$ and $\mathrm{PA}=57^{\circ}$. G1 has an absolute $B$-band magnitude of $M_{B}=-20.5$ which translates to $L_{B}=0.39 L_{B}^{*}$. The galaxy is at a projected distance of $D=26.9 \mathrm{kpc}$ from the quasar.

The spatial radial velocity of $\mathrm{G} 1$, as derived from [O II], is presented in Figure 4(b). The data suggest that G1 exhibits more of a global shear than rotation. The maximum observed shear velocity is $\sim 42 \mathrm{~km} \mathrm{~s}^{-1}$. Below the velocity curve, the $\mathrm{Mg}$ II and the $\mathrm{Mg}$ I absorption profiles are shown on the same velocity scale. The mean absorption redshift is offset by $-16 \mathrm{~km} \mathrm{~s}^{-1}$ from the galaxy systemic velocity. The $\mathrm{Mg}$ II absorption is a single kinematic component having a velocity spread of roughly $282 \mathrm{~km} \mathrm{~s}^{-1}$. Most of the component is composed of highly saturated clouds. From the $\mathrm{Mg}$ I profile, one can resolve the individual clouds that are saturated in $\mathrm{Mg}$ II. The galaxy velocities are consistent with the velocities of the absorbing gas. However, there is a large amount of $\mathrm{Mg}$ II gas that has 
(b)
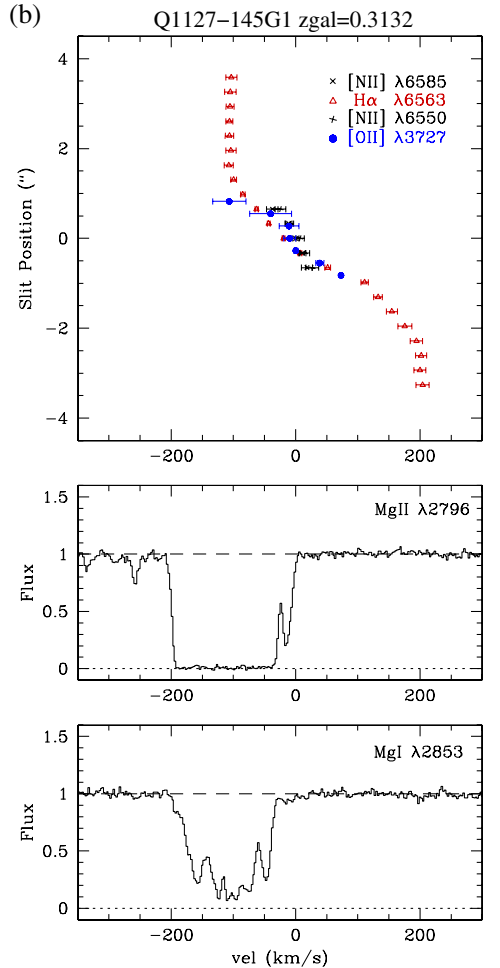

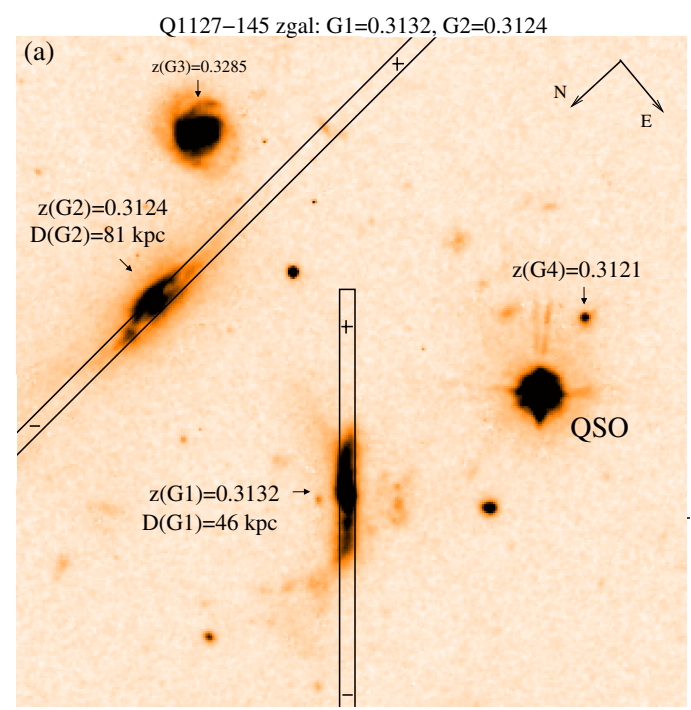

(c)
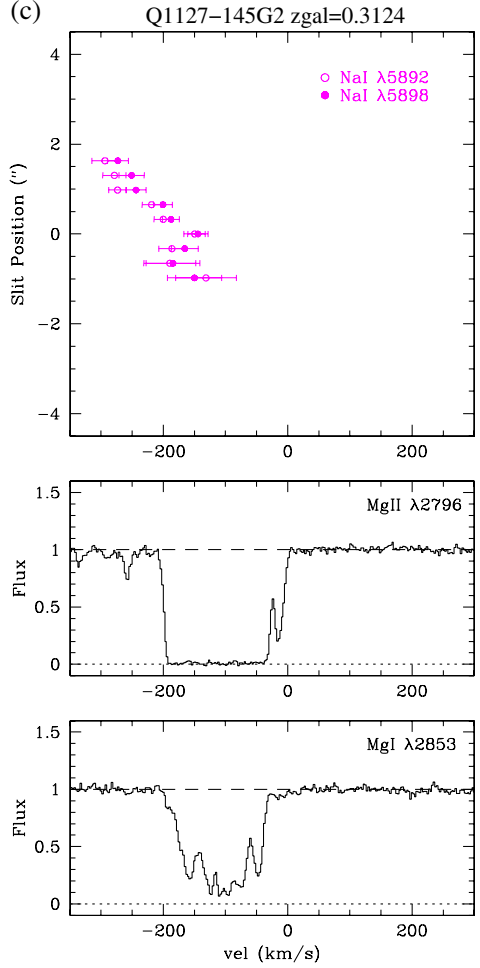

Figure 5. (a) Same as Figure 2(a) except that this is a $30^{\prime \prime} \times 30^{\prime \prime} F 814 \mathrm{~W}$ WFPC-2/HST image of the quasar field Q1127-145. The two targeted spiral galaxies G1 and $\mathrm{G} 2$ have redshifts of $z=0.3132$ and $z=3124$, respectively. G1 and $\mathrm{G} 2$ are at impact parameters of $D=45.6 \mathrm{kpc}$ and $D=81.0 \mathrm{kpc}$, respectively. (b) Same as Figure 2(b) except G1 in the Q1127-145 field. (b) Same as Figure 2(b) except G2 in the Q1127-145 field.

(A color version of this figure is available in the online journal.)

greater velocities than those of the galaxy. The bulk of the more neutral/high column density gas, as indicated by $\mathrm{Mg} \mathrm{I}$, is at the galaxy systemic velocity.

\section{6. $Q 1127-145$ G3}

A WFPC-2/HST image of the Q1127-145 field is shown in Figure 4(c). We present a newly identified galaxy, G3, at $z=0.3285$. The galaxy was identified by $\mathrm{H} \alpha$ and [N II] emission lines. The absorption was also recently discovered, and is presented here in Figure 4(d). This weak system has an equivalent width of $W_{r}(2796)=0.029 \AA$.

The face-on galaxy has an inclination of $i=1^{\circ}$ with a PA $\sim 69^{\circ}$ (the PA is highly uncertain since $i \simeq 0$ ). The galaxy has a large bar with a sizable bright bulge; similar to a local $\mathrm{SBb}$ galaxy. It has absolute magnitude of $M_{B}=-20.51$ which translates to $L_{B}=0.60 L_{B}^{*}$. The galaxy is at a projected distance of $D=91.4 \mathrm{kpc}$ from the quasar.

The rotation curve of G3 is presented in Figure 4(d). The maximum observed rotational velocity is $\sim 80 \mathrm{~km} \mathrm{~s}^{-1}$. Given that significant rotation is observed, the galaxy is most likely not completely face-on as the GIM2D model inclination suggests. Below the rotation curve, the $\mathrm{Mg}$ II and the $\mathrm{Mg}_{\mathrm{I}}$ absorption profiles are shown on the same velocity scale. The absorption redshift is offset by $-46 \mathrm{~km} \mathrm{~s}^{-1}$ from the galaxy systemic velocity. The $\mathrm{Mg}$ II absorption contains two separate single cloud components. Both clouds have a velocity spread of $\sim 15 \mathrm{~km} \mathrm{~s}^{-1}$ and are separated by $125 \mathrm{~km} \mathrm{~s}^{-1}$. No significant $\operatorname{Mg}_{\mathrm{I}}(3 \sigma$, $\left.W_{r} \leqslant 0.003 \AA\right)$ is detected. The projected galaxy rotation velocities are consistent with both cloud velocities; each cloud aligns with each side of the rotation curve.

\section{7. $Q 1127-145 G 1, G 2$}

The history of the Q1127-145 $z=0.312710$ absorption system is quite complex. The Mg II absorption was initially detected by Bergeron \& Boissé (1991) and was determined to be a DLA (see Rao \& Turnshek 2000) since HST UV data show a damped Ly $\alpha$ with $N_{\mathrm{HI}}=5.1 \times 10^{21} \mathrm{~cm}^{-2}$ (Lane et al. 1998). The equivalent width of the system is $W_{r}(2796)=1.773 \AA$.

The true identity of the absorbing galaxy has been a topic of debate in the literature. A WFPC-2/HST image of the Q1127-145 field is shown in Figure 5(a). Bergeron \& Boissé (1991) spectroscopically identified G1 and G2 to be at the redshift of the absorption. G1 exhibits strong emission lines and G2 has no detectable emission lines. G1 was assumed to be the absorbing galaxy, since it is closer to the quasar and has significant star formation (Bergeron \& Boissé 1991). Lane et al. (1998) later spectroscopically identified G4 via an [O III] doublet with $z=0.3121$, which was also consistent with the absorption redshift and was assumed to be the absorber only due to its proximity to the quasar. Lane et al. (1998) state that it is also possible that the three galaxies may have undergone a strong interaction where the absorption could arise from tidal debris. In Figure 5(a), it is apparent from the tidal disturbances that G1 has undergone interactions in the past. G2 also exhibits some tidal material to the north of the galaxy (which is less apparent in the figure).

Rao et al. (2003) and Nestor et al. (2002) suggest, from ground-based multi-band imaging, that the low surface brightness emission detected around the quasar (see Figure 5(a)) could arise from a foreground low surface brightness galaxy at the absorption redshift. However, it is possible that the low 

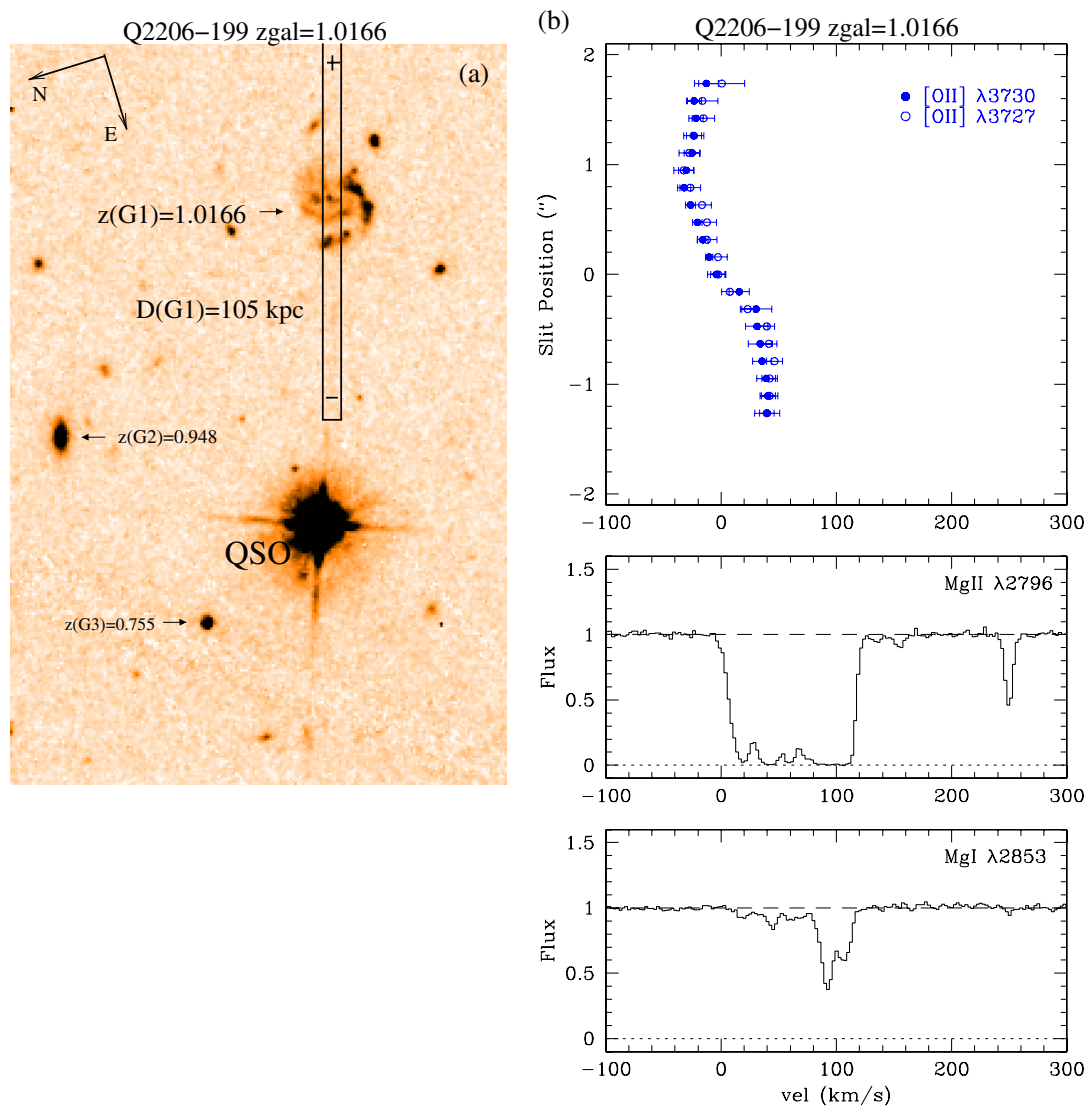

Figure 6. (a) Same as Figure 2(a) except for the quasar field Q2206-199. The target spiral galaxy G1 is at a redshift of $z=1.10166$ at $D=104.6 \mathrm{kpc}$. (b) Same Figure 2(b) except G1 in the Q2206-199 field.

(A color version of this figure is available in the online journal.)

surface brightness signal is coming from the quasar host galaxy at $z_{\mathrm{em}}=1.18$. The background quasar is radio-loud and has strong X-ray emission. An X-ray jet extends northeast $236 \mathrm{kpc}$ from the quasar, projected (Siemiginowska et al. 2002, 2007). There is a diffuse halo, both detected in radio and X-ray, around the host quasar. It is not yet clear whether the X-ray halo is real or a result of blurring from the instrument PSF (A. Siemiginowska 2007, private commutation). Thus, it is possible that the low surface brightness detected by Rao et al. (2003) may be from the background quasar. Chun et al. (2006) found a possible underlying galaxy 0.'6 from the quasar. Again, this may be a foreground galaxy, or structure from the quasar host galaxy which is commonly observed (e.g., Bahcall et al. 1997).

Galaxies G1 and G2 were targeted for this study. These galaxies are spatially separated by only $55.3 \mathrm{kpc}$ projected, and by a line-of-sight velocity of $183 \mathrm{~km} \mathrm{~s}^{-1}$. G1 is an almost edge-on $\left(i=82^{\circ}\right)$ spiral that displays asymmetries on both sides of the galaxy. The galaxy has $\mathrm{PA}=21^{\circ}$. It has absolute magnitude of $M_{B}=-20.40$, which translates to $L_{B}=0.54 L_{B}^{*}$. The galaxy is at a projected distance of $D=45.6 \mathrm{kpc}$ from the quasar line of sight. G2 is an interesting galaxy; it has a major dust lane and a large bulge. Given that we detected no emission lines, this galaxy could either be classified as $\mathrm{Sa}$ or as an early-type S0 galaxy. G2 also has a tidal disturbance along the major axis of the galaxy toward the north. The galaxy has an inclination of $i=73^{\circ}$ and $\mathrm{PA}=61^{\circ}$. It has absolute magnitude of $M_{B}=-20.57$ which translates to $L_{B}=0.63 L_{B}^{*}$. The galaxy is at a projected distance of $D=81.0 \mathrm{kpc}$ from the quasar.

The rotation curve of $\mathrm{G} 1$, obtained from the [O $\mathrm{II}], \mathrm{H} \alpha$, and the $[\mathrm{N}$ II] doublet, is presented in Figure 5(b). The maximum observed rotational velocity is $204 \mathrm{~km} \mathrm{~s}^{-1}$. The rotation curve of G2, obtained from the Na I absorption doublet, is presented in Figure 5(c). The maximum observed rotational velocity of G2 is $90 \mathrm{~km} \mathrm{~s}^{-1}$. Below both rotation curves, the $\mathrm{Mg}$ II and the $\mathrm{Mg}$ I absorption profiles are shown on the velocity scale defined by $\mathrm{G} 1$. The mean absorption redshift is offset by $-112 \mathrm{~km} \mathrm{~s}^{-1}$ from the systemic velocity of G1. The Mg II absorption can be broken up into two kinematic components. The first large saturated component has a velocity spread of roughly $235 \mathrm{~km} \mathrm{~s}^{-1}$. The second component, blueward of the main component, contains a few weak clouds and has a velocity spread of roughly $68 \mathrm{~km} \mathrm{~s}^{-1}$. In the $\mathrm{Mg}$ I profile, one can resolve the individual clouds that are saturated in $\mathrm{Mg}$ II. Almost all of the $\mathrm{Mg}_{\mathrm{I}}$ gas is aligned with the saturated $\mathrm{Mg}$ II component. Only a very weak $\mathrm{Mg}$ I cloud is detected in a second kinematic component. The main component of the $\mathrm{Mg}$ II gas aligns with the blueward wing of the $\mathrm{G} 1$ rotation curve, which is on the side toward the quasar. The Mg II absorption velocities start at the systemic velocity of G1 and is centered on the maximum galaxy rotation velocity. Since the rotation curve of $\mathrm{G} 2$ is derived from $\mathrm{Na}$ I absorption lines, it is likely that the maximum rotation speed of the galaxy extends to larger velocities then detected. The velocities of G2 are consistent a portion of the large saturated component of the $\mathrm{Mg}$ II and the small blueward clouds.

\section{8. $Q 2206-199$ G1}

A WFPC-2/HST image of the Q2206-199 field is shown is Figure 6(a). The $z=1.0166$ galaxy, G1, was targeted for this study. The galaxy was spectroscopically identified by 
Bergeron et al. (1992). The Mg II absorption was first reported by Sargent et al. (1988). The HIRES/Keck spectrum was originally presented by Prochaska \& Wolfe (1997). The Mg II equivalent width is $W_{r}(2796)=1.057 \AA$.

The spiral galaxy G1 is quite unusual in brightness and morphology. The galaxy has an absolute magnitude of $M_{B}=$ -23.8 which translates to $L_{B}=8.0 L_{B}^{*}$. Galaxies with super$L^{*}$ luminosities are quite rare and represent only a few percent of the galaxy population. The galaxy spiral structure is tightly wound with a large leading arm. There appears to be numerous $\mathrm{H}$ II regions. The bulge is compact and offset from the isophotal center. Note that at the galaxy redshift, the rest-frame mean wavelength of the $F 702 W$ filter is around $3440 \AA$, roughly restframe $U$ band. The galaxy inclination is $i=57^{\circ}$ with a PA $=$ $67^{\circ}$. The galaxy is at a projected distance of $D=104.6 \mathrm{kpc}$ from the quasar.

The rotation curve, obtained from the [O II] doublet, is presented in Figure 6(b). The maximum observed rotational velocity is $\sim 40 \mathrm{~km} \mathrm{~s}^{-1}$. Given the low observed rotation velocities, the galaxy is likely more face-on than the GIM2D model inclination suggests. The galaxy morphology is asymmetric and unusual which makes it difficult to determine the inclination. Below the rotation curve, the $\mathrm{Mg}$ II and the $\mathrm{Mg}$ I absorption profiles are shown on the same velocity scale. The mean absorption redshift is offset by $73 \mathrm{~km} \mathrm{~s}^{-1}$ from the galaxy systemic velocity. The $\mathrm{Mg}$ II absorption can be broken up into two kinematic components. The first one has velocity spread of roughly $150 \mathrm{~km} \mathrm{~s}^{-1}$ and is mostly saturated. The second component is a single cloud with a velocity spread of roughly $28 \mathrm{~km} \mathrm{~s}^{-1}$ that is offset $198 \mathrm{~km} \mathrm{~s}^{-1}$ redward of the main component velocity center. From the $\mathrm{Mg}$ I profile, one can resolve the individual clouds that are saturated in Mg II. Most of the Mg I gas is shifted $100 \mathrm{~km} \mathrm{~s}^{-1}$ from the systemic velocity of the galaxy. The redward arm the rotation curve aligns with only a small portion of velocity of the main $\mathrm{Mg}$ II component. However, there is a large amount of $\mathrm{Mg}$ II gas that has greater velocities than those of the galaxy. The bulk of the more neutral/high column density gas, as indicated by $\mathrm{Mg}$ I, has velocities greater than that of the observed galaxy rotational velocities.

\subsection{Summary I: Observational Kinematic Comparisons}

In our direct comparison of galaxy disk and halo gas kinematics, traced by $\mathrm{Mg}$ II and $\mathrm{Mg}$ I absorption, we find the following: (1) in all 10 cases, the observed galaxy rotation velocities show substantial overlap with the bulk of the absorption velocities, (2) in seven of 10 cases, the $\mathrm{Mg}$ II and $\mathrm{Mg}$ I absorption resides to one side of the galaxy systemic velocity. In the remaining cases (Q0002+051 G1, Q0836+113 G1, and Q1127-145 G3), absorption resides on both sides of the galaxy systemic velocity. Our findings are similar to those of Steidel et al. (2002), even though their sample targeted highly inclined and edge-on disk galaxies with $\mathrm{PA} \sim 0$. Here, we have attempted to select galaxies with a range of inclination and PA with respect to the quasar line of sight (see Table 6).

To see if there are differences in the $\mathrm{Mg}$ II absorption profiles as a function of inclination, we have separated the galaxies into two inclination bins with $i<60^{\circ}$ (five galaxies) and $i>60^{\circ}$ (six galaxies). The galaxies from the Steidel et al. (2002) sample are included here. For this comparison, multiple galaxies that can be associated with a single absorption system (such as G1 and $\mathrm{G} 2$ in Q0450-132 and 1127-145) were removed since we cannot confidently know whether one or both galaxies host the $\mathrm{Mg}$ II absorption.

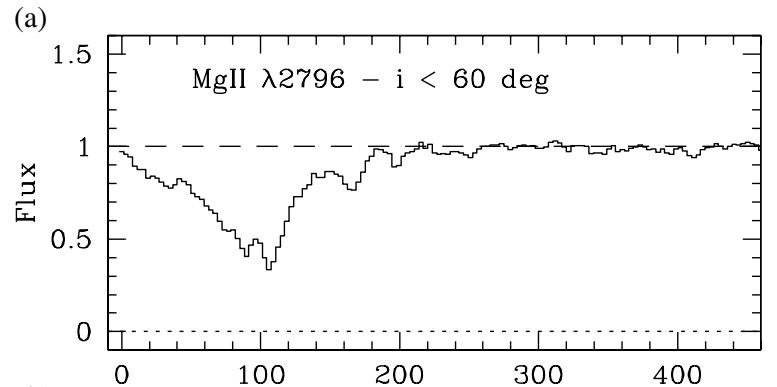

(b)

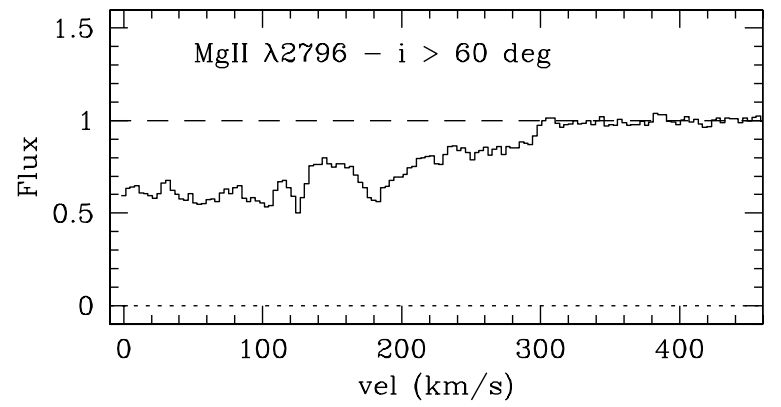

Figure 7. Combined Mg II absorption spectra for galaxies separated into two inclination bins. The spectra are plotted vs. absolute velocity difference from the galaxy systemic velocity. The five galaxies of Steidel et al. (2002) are included. The two galaxy pairs, G1 and G2 of Q0450-132 and Q1127-145, are excluded here. (a) The summed spectra for five galaxies with $i<60^{\circ}$. (b) The summed spectra for six galaxies with $i>60^{\circ}$.

In Figure 7, we show the co-added Mg II absorption profiles. The data are plotted as an absolute velocity difference from the galaxy systemic velocity. As seen in Figure 7(a), the combined spectra of the galaxies with $i<60^{\circ}$ show that absorption resides between 0 and $180 \mathrm{~km} \mathrm{~s}^{-1}$ with a peak in the optical depth at $v \sim 100 \mathrm{~km} \mathrm{~s}^{-1}$. In Figure 7(b), the combined spectra show that galaxies with $i>60^{\circ}$ are associated with $\mathrm{Mg}$ II absorption with smoothly varying optical depth spread over $\sim 300 \mathrm{~km} \mathrm{~s}^{-1}$. A Kolmogorov-Smirnov $(\mathrm{K}-\mathrm{S})$ test reveals the probability of the two optical depth distributions being drawn from the same sample is $P_{\mathrm{KS}}=0.000873$. This rules out the null hypothesis of similar $\mathrm{Mg}$ II optical depth distributions as a function of inclination at the $99.91 \%$ confidence level $(3.3 \sigma)$. The data are suggestive that $\mathrm{Mg}$ II absorption velocity spread may be a function of galaxy orientation; galaxies with higher inclination have $\mathrm{Mg}$ II absorption with larger velocity spreads and more evenly distributed optical depths (on average at any given velocity). However, since the number of galaxies per inclination bin is small, we need to acquire a larger sample to see if the trend holds.

A trend with inclination might be expected if the absorbing gas kinematics is well represented by a monolithic rotating halo. Mg II absorption velocity spreads in four out of the five galaxies in the Steidel et al. (2002) sample were shown to be consistent with a monolithic rotating halo model that allowed for lagging rotation with increasing height above the disk. Given the apparent kinematic trend with inclination, we investigate whether the lagging halo model (Steidel et al. 2002) can successfully predict the Mg II absorption velocity spreads of our 10 galaxies.

\section{GALAXY KINEMATICS AND HALO-DISK MODELS}

We apply the simple halo model of Steidel et al. (2002) to our systems in order to determine whether an extended disk-like 
Table 6

Galaxy Disk Model Input Values

\begin{tabular}{lccccc}
\hline \hline QSO Field & $\begin{array}{c}\text { Galaxy } \\
\text { ID }\end{array}$ & $\begin{array}{c}D \\
(\mathrm{kpc})\end{array}$ & $\begin{array}{c}v_{\max } \\
\left(\mathrm{km} \mathrm{s}^{-1}\right)\end{array}$ & $\begin{array}{c}i \\
(\mathrm{deg})\end{array}$ & $\begin{array}{c}\text { PA } \\
(\mathrm{deg})\end{array}$ \\
\hline Q0002+051 & G1 & $25.9 \pm 0.5$ & -49 & $38_{-31}^{+12}$ & $43_{-6}^{+14}$ \\
Q0229+131 & G1 & $37.5 \pm 0.5$ & -281 & $58_{-1}^{+2}$ & $22_{-2}^{+2}$ \\
Q0450-132 & G1 & $50.1 \pm 0.4$ & -98 & $66_{-2}^{+3}$ & $42_{-3}^{+2}$ \\
Q0450-132 & G2 & $62.7 \pm 0.7$ & 47 & $75_{-2}^{+2}$ & $54_{-2}^{+2}$ \\
Q0454-220 & G1 & $107.9 \pm 0.8$ & 138 & $41_{-2}^{+1}$ & $76_{-2}^{+1}$ \\
Q0836+113 & G1 & $26.9 \pm 0.9$ & -42 & $78_{-1}^{+1}$ & $57_{-1}^{+1}$ \\
Q1127-145 & G1 & $45.6 \pm 0.3$ & 204 & $82_{-0}^{+0}$ & $21_{-0}^{+0}$ \\
Q1127-145 & G2 & $81.0 \pm 0.3$ & -90 & $73_{-3}^{+1}$ & $61_{-2}^{+2}$ \\
Q1127-145 & G3 & $91.4 \pm 0.2$ & -80 & $1_{-1}^{+3}$ & $69_{-19}^{+34}$ \\
Q2206-199 & G1 & $104.6 \pm 1.4$ & -40 & $57_{-14}^{+5}$ & $67_{-6}^{+7}$ \\
\hline
\end{tabular}

rotating halo is able to reproduce all or most of the observed $\mathrm{Mg}$ II absorption velocity spread. The model is a co-rotating disk with velocity decreasing as a function of scale height.

The line-of-sight velocity, $v_{\text {los }}$, predicted by this disk halo model is a function of the measurable quantities $D, i$, PA (the angle between the galaxy major axis and the quasar line of sight), and $v_{\max }$, which is the maximum projected galaxy rotation velocity,

$$
\begin{gathered}
v_{\mathrm{los}}=\frac{-v_{\max }}{\sqrt{1+\left(\frac{y}{p}\right)^{2}}} \exp \left\{-\frac{\left|y-y_{\circ}\right|}{h_{v} \tan i}\right\} \text { where, } \\
y_{\circ}=\frac{D \sin \mathrm{PA}}{\cos i} \text { and } p=D \cos \mathrm{PA},
\end{gathered}
$$

where the free parameter, $h_{v}$, is the lagging gas velocity scale height and where $y$ is the projected line-of-sight position above the disk plane. The parameter $y_{\circ}$ represents the position at the projected mid-plane of the disk. The range of $y$ values is constrained by the model disk-halo thickness, $H_{\text {eff }}$, such that $y_{\circ}-H_{\text {eff }} \tan i \leqslant y \leqslant y_{\circ}+H_{\text {eff }} \tan i$. The distance along the line of sight relative to the point where it intersects the projection of the disk mid-plane is then $D_{\text {los }}=\left(y-y_{\circ}\right) / \sin i$. There are no assumptions about the spatial density distribution of $\mathrm{Mg}$ II absorbing gas, except that $H_{\text {eff }}$ is the effective thickness of the gas layer capable of giving rise to absorption.

In order to maximize the rotational velocity predicted by the model, we assume $h_{v}=1000 \mathrm{kpc}$, which effectively removes the lagging halo velocity component (such that the exponential in Equation (1) is roughly equal to unity).

In Figures 8(a)-(h), we show the Mg II absorption profiles for each galaxy, where the shaded regions indicate detected absorption. Below each absorption profile is the disk halo model velocities as a function of $D_{\text {los }}$ derived for each galaxy (solid line) using Equation (1) and parameters in Table 6. Recall that, at $D_{\text {los }}=0 \mathrm{kpc}$, the model line of sight intersects the projected mid-plane of the galaxy. The dashed curves represent the disk halo model velocities derived from the combination of the minimum and maximum uncertainties in the PA and $i$. In some cases (see Figure 8(b)) the values of the PA and $i$ are well determined such that the dashed curves lie on the solid curves. The model also predicts the line-of-sight position, $D_{\text {los }}$, of the halo gas at each velocity, $v_{\text {los }}$.

The disk halo model is successful at predicting the observed $\mathrm{Mg}$ II absorption velocity distribution when the solid (or dashed) curves span the same velocity spread as that of the Mg II absorption gas. The model curves must occupy the full shaded region to be $100 \%$ successful. If this is not the case, one can conclude that disk-like halo rotation is not the only dynamic mechanism responsible for the Mg II kinematics. In the following subsections, we discuss the disk model of the individual galaxies.

\section{1. $Q 0002+051 G 1$}

The galaxy G1 exhibits a low level velocity shear. Given the velocity spread of the gas $\left(\sim 475 \mathrm{~km} \mathrm{~s}^{-1}\right)$, it is impossible for the bulk of the absorption gas to be consistent with the observed velocities of G1. In Figure 8(a), we see that the galaxy disk halo model is counterrotating with respect to the dominate saturated $\mathrm{Mg}$ II component. There is no overlap between the predicted halo model velocities with those of the $\mathrm{Mg}$ II and $\mathrm{Mg}$ I absorption. Even if the galaxy had a highly significant velocity shear, the bulk of the Mg II clouds would not be consistent in velocity space. Given the number of high velocity components, it is unclear that this absorption profile represents a gravitationally bound gaseous galactic halo.

\section{2. $Q 0229+131 G 1$}

The galaxy G1 has an asymmetric rotation curve with the largest rotation velocity observed in the direction of the quasar line of sight. In Figure 8(b), we see that the disk halo model velocities are consistent with the bulk of the $\mathrm{Mg}$ II absorption. Thus, extended disk-like halo rotation could be invoked to explain most of the observed halo gas velocities. However, there remains a small single $\mathrm{Mg}$ II halo cloud $\sim 400 \mathrm{~km} \mathrm{~s}^{-1}$ from systemic that cannot be explained by halo rotation alone. This suggests other dynamic processes give rise to some of the $\mathrm{Mg}$ II absorption.

\section{3. $Q 0450-132 G 1, G 2$}

Galaxies G1 and G2 are potentially interacting galaxies, as evident from their morphologies and strong emission lines. It is possible that these interactions are an effective mechanism in producing extended $\mathrm{Mg}$ II absorption in the halo (Bowen et al. 1995; Kacprzak et al. 2007; Rubin et al. 2009). In Figure 8(c), we plot the disk halo models for both galaxies. G1, the galaxy closest to the quasar, has model halo gas kinematics that are counterrotating with respect to the $\mathrm{Mg}$ II absorption. G2, on the other hand, has modeled halo velocities that are consistent with those of the Mg II absorption. The halo model of G2 is also consistent with the bulk of the $\mathrm{Mg}$ I. Given that G1 was observed along the major axis and G2 was not, if we assume that $\mathrm{G} 2$ had comparable rotation speeds as G1, the halo model velocities would overlap with most of the absorption velocities. We will discuss the difficulties of disentangling these multiple galaxy systems in Section 4.9.

\section{4. $Q 0454-220$ G1}

Galaxy G1 has a symmetric rotation curve that completely flattens out at the maximum velocity of $138 \mathrm{~km} \mathrm{~s}^{-1}$. In Figure 8(d), we see that the disk halo model has velocities consistent with the bulk of the Mg II absorption velocities. They are also consistent with the $\mathrm{Mg}$ I absorption velocities. However, there is an inconsistency of $\sim 40 \mathrm{~km} \mathrm{~s}^{-1}$ between the halo model and $\mathrm{Mg}$ II absorption velocities. Thus, the halo model is unable to reproduce the total observed spread of $\mathrm{Mg}$ II absorption velocities. 

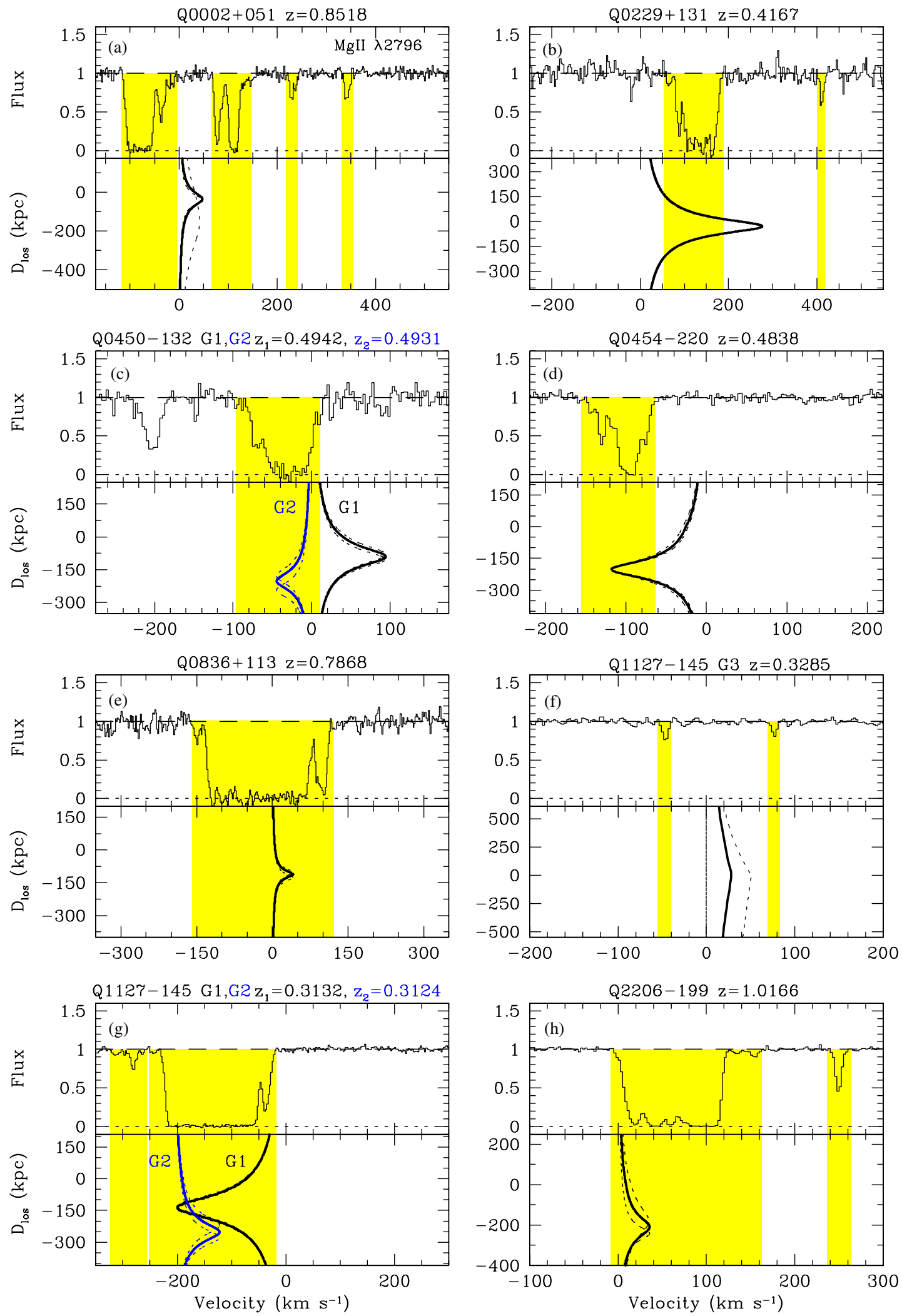

Figure 8. $\mathrm{Mg}$ II absorption profiles and the disk model velocities as a function of $D_{\mathrm{los}}$ (solid curve) are shown for each galaxy in the top and bottom panels, respectively. The Mg II absorption velocities are shaded in. The solid curve is computed using Equation (1) and the values from Table 6. The dashed curves are models computed for the maximum and minimum predicted model velocities given the uncertainties of $i$ and PA. The disk model is successful and reproducing the observed absorption velocities in the solid curve overlaps with the entire shaded region. $D_{\text {los }}$ is equal to zero when the quasar line of sight intersects the projected mid-plane of the galaxy. The panels are as follows: (a) Q0002+051 G1, G1, (b) Q0229+131 G1, (c) Q0450-132 G1 and G2, (d) Q0454-220 G1, (e) Q0836+113 G1, (f) Q1127-145 G3, (g) Q1127-145 G1 and G2, and (h) Q2206-199 G1.

(A color version of this figure is available in the online journal.) 


\section{5. $Q 0836+113 G 1$}

The galaxy G1 exhibits minimal rotation; the velocities are more indicative of a global shear. The galaxy systemic velocity is centered roughly in the middle of the $\mathrm{Mg}$ II and the bulk of the $\mathrm{Mg}$ I absorption profiles. In Figure 8(e), we see that the halo model also shows little rotation, roughly $50 \mathrm{~km} \mathrm{~s}^{-1}$. If the model was a true representation of the halo, then more than $50 \%$ of the absorbing gas has velocities inconsistent with disk rotation that are larger than the model velocities. Even if G1 has a more significant velocity shear, the model would still not be able to explain the gas blueward of the galaxy systemic velocity. Here, the models fails to predict the bulk of the absorption velocities.

\section{6. $Q 1127-145$ G3}

The galaxy G3 appears face-on, however, it exhibits a maximum rotation of $80 \mathrm{~km} \mathrm{~s}^{-1}$. The model inclination of $1^{\circ}$ is likely incorrect given the observed rotation velocities. In Figure 8(f), the disk halo model for G3 exhibits little lineof-sight velocity $\left(\sim 20-50 \mathrm{~km} \mathrm{~s}^{-1}\right)$ given its orientation with respect to the quasar line of sight. Given the model parameters listed in Table 6, we varied the galaxy inclination, such that $0^{\circ} \leqslant i \leqslant 45^{\circ}$, in an attempt to reproduce the observed absorption velocities. Even with $i=45^{\circ}$, the halo model fails to reproduce the observed $\mathrm{Mg}$ II absorption velocities. For the disk halo scenario, the halo gas is expected to reside to one side of the rotation curve. The nature of this Mg II absorption profile is interesting; two weak clouds separated by $125 \mathrm{~km} \mathrm{~s}^{-1}$. It is likely that these clouds could arise in either a patchy diffuse halo or the line of sight is intercepting small-scale structure near the galaxy halo. In any case, the model is unable to reproduce the observed $\mathrm{Mg}$ II absorption velocities.

\subsection{Q1127-145 G1, G2}

Galaxies G1 and G2 are potentially interacting galaxies, as evident from their morphologies. These galaxies appear to be in a small group including G4. In Figure 8(g), we plot the disk halo models for G1 and G2. The model for G1 has velocities that are consistent with up to $200 \mathrm{~km} \mathrm{~s}^{-1}$ spread of the saturated component of the $\mathrm{Mg}$ II and all of the $\mathrm{Mg}$ I absorption. The disk halo model of G2 is counterrotating with respect to $\mathrm{G} 1$ as viewed from the quasar line of sight. The model velocities are consistent with the remaining absorption of the saturated component which was not covered by G1. The model velocities of both G1 and G2 overlap $\sim 75 \mathrm{~km} \mathrm{~s}^{-1}$. The second weaker kinematic component, $\sim 300 \mathrm{~km} \mathrm{~s}^{-1}$ blueward of systemic velocity of G1, cannot be explained given the predicted halo velocities. It is possible that the saturated component of the $\mathrm{Mg}$ II absorption could arise from either G1, G2, or both. The weaker component may arise from tidal debris stirred up by the apparent interactions. It is also possible that some of the $\mathrm{Mg}$ II absorption is associated with G4. It is likely that the saturated component is associated with a nearby galaxy since it is a DLA and many other low ions have also been detected. We will discuss the difficulties of disentangling these multiple galaxy systems in Section 4.9. In any case, the model is mostly successful, except that it does not reproduce all of the $\mathrm{Mg}$ II absorption velocities.

\subsection{Q2206-199 G1}

The G1 galaxy is the largest in our sample, and the second furthest away from the quasar line of sight. In Figure 8(h), the disk halo model shown has very little line-of-sight velocity $\left(\sim 40 \mathrm{~km} \mathrm{~s}^{-1}\right)$. It is clear that the model of this moderately inclined galaxy does not reproduce the observed absorption velocities. In fact, the bulk of the $\mathrm{Mg}$ II and $\mathrm{Mg}_{\mathrm{I}}$ has velocities $\sim 50 \mathrm{~km} \mathrm{~s}^{-1}$ greater than the galaxies maximum observed rotation velocity. This is peculiar, since the dominant saturated component is commonly expected to be associated with the galaxy disk, yet the kinematics here suggest otherwise. This galaxy-absorber pair is a another example demonstrating that disk-like halo rotation cannot be the only mechanism driving the kinematics of halo gas.

\subsection{Mg II Absorption from Galaxy Pairs/Groups}

Since $\mathrm{Mg}$ II absorbers were first associated with galaxy halos, it has been common practice to associate one galaxy with an absorption system at a given redshift (e.g., Bergeron \& Boissé 1991; Steidel et al. 1994; Churchill et al. 1996; Guillemin \& Bergeron 1997). It is now becoming evident that $\mathrm{Mg}$ II absorption also arises in small groups of galaxies (Bowen et al. 1995; Churchill \& Charlton 1999) and even clusters (Lopez et al. 2008). In this paper, we present two such examples: Q0450-132 G1, G2 and Q1127-145 G1, G2, G4. The pair of galaxies in Q0450-132 are close to each other in both projected distance and velocity. Both galaxies have morphological evidence (one-sided tidal tails) that is classically associated with interacting/merging galaxies. The Q1127-145 field contains three galaxies at similar redshift (we have recently spectroscopically identified two additional galaxies with similar redshifts within $D=250 \mathrm{kpc}$ from the quasar; G. G. Kacprzak et al. 2010, in preparation). The two galaxies studied here, G1 and G2, have evidence of morphological perturbations and extended tidal material.

The Q0450-132 and Q1127-145 galaxy pairs have observed rotation velocities that overlap with those of the $\mathrm{Mg}$ II absorption. Given these two fields, it is clear that it can be difficult at times to associate one particular galaxy with an absorption system. One alternative interpretation is that the material responsible for the absorption is tidal debris originating from both galaxies due to past mergers and harassments (similar to the Magellanic stream). Given that the galaxies are in close proximity (projected), it is also possible that these galaxies share a common gas structure that gives rise to the absorption.

\subsection{Summary II: Disk Halo Model}

In an effort to reproduce the $\mathrm{Mg}$ II absorption velocities, we used a disk halo model to compute the expected absorption velocities. In only one case, Q0229+131 G1, we were able to reproduce the full spread of the $\mathrm{Mg}$ II absorption velocities in a disk halo model. In four other cases, Q0454-220, Q0450-132 (G2), and Q1127-145 (G1 and G2), the velocity region with the strongest absorption is consistent with the model. However, the halo model of the galaxy G2 toward Q0450-132 does not account for roughly $35 \%$ of the absorption. In the cases of both Q0229+131 and Q1127-145, the models cannot account for the unsaturated small cloud structures at higher velocities relative to systemic. For each case, the models do reproduce some of the absorption velocities, however, the disk rotating halo model is insufficient to account for the full observed $\mathrm{Mg}$ II absorption velocity range.

We emphasize that the disk halo model applied to the data is an extreme version of the spatial-kinematic relationship in that (1) all the gas is assumed to rotate at the maximum observed velocity of the galaxy and (2) the scale height of the models 
$\left(h_{v}=1000 \mathrm{kpc}\right)$ is highly unrealistic. These extreme conditions were required in order to obtain the greatest degree of agreement between the model and the data. If these conditions are relaxed, the level of agreement we found is diminished substantially. None the less, even under these extreme and unrealistic model parameters, the disk halo model provides insight into the degree at which rotation kinematics can account for limited regions of the absorption velocity spread.

What we learn from the disk halo model is that it is reasonable to suggest that additional dynamical processes (such as infall, outflow, supernovae winds, mergers, etc.) and/or additional satellites or unidentified galaxies giving rise to some of the Mg II absorption contribute to the observed velocity spreads. The possibility of unidentified galaxies is difficult to quantify, for the assigning of a given galaxy to a given absorption system is by its very nature not $100 \%$ certain. It would, however, require the galaxies we have assigned to not be a significant contributor to the absorption and/or the unidentified galaxy to have very extended absorbing gas. The former statement is not strongly supported by a body of previous studies (e.g., Bergeron \& Boissé 1991; Le Brun et al. 1993; Steidel et al. 1994; Guillemin \& Bergeron 1997; Steidel et al. 1997; Churchill et al. 2005; Tripp \& Bowen 2005; Zibetti et al. 2007; Chen \& Tinker 2008; Tinker $\&$ Chen 2008). The later statement is based upon the detected galaxies in the quasar fields. The statement would not apply to putative galaxies below our detection limit that are in close projection to the quasar.

\section{5. $\Lambda$ CDM COSMOLOGICAL GALAXY SIMULATIONS}

To further understand the halo gas dynamics and the mechanisms driving the observed gas velocity spread, we investigate high-resolution cosmological simulation of galaxy formation, which include the dynamical processes of infall, outflow, supernovae winds, mergers, etc. Simulations provide the only theoretical means to fully incorporate these dynamical processes in a cosmological setting. We use the method of quasar absorption lines through the simulations to "observe" the Mg II absorption kinematics. Here, we analyze a single $z=0.9$ simulated galaxy in detail to study the possible structures and mechanisms that give rise to the observed $\mathrm{Mg}$ II halo gas kinematics. By comparing halo gas kinematics in the simulations to the spatial and dynamic processes of the simulated galactic environments, we can gain further insights into the observed $\mathrm{Mg}$ II absorption properties.

\subsection{Description of the Simulations}

The $\Lambda$ CDM cosmological simulations are performed using the Eulerian Gasdynamics plus $N$-body Adaptive Refinement Tree (ART) code (Kravtsov 1999, 2003). Physical processes implemented in the code include star formation and stellar feedback, metal enrichment from type II and Ia supernovae, self-consistent advection of metals, and metallicity-dependent cooling, and photoionization heating due to a cosmological ultraviolet background (Haardt \& Madau 1996). The code does not include radiative transfer, magnetic fields, or KelvinHelmholtz instabilities. The cosmological model has $\Omega_{m}=$ $0.3, \Omega_{\Lambda}=0.7, h=0.7$, and $\sigma_{8}=0.9$.

These simulations have high star formation efficiency at the resolution scale. This assumption is motivated by studies of star formation in simulations of isolated disks with a similar resolution (Tasker \& Bryan 2006). The gas consumption timescale is $\sim 10^{7} \mathrm{yr}$ for the star-forming cells, however, only a small fraction $(\sim 1 \%)$ of the gas in the disk is forming stars in a typical time step of $\sim 40 \mathrm{Myr}$ at $z \sim 1$. As a result, the disk-averaged star formation efficiency is low: the gas consumption timescale is $\sim 10^{9} \mathrm{yr}$, consistent with observations (Kennicutt 1998).

The computational region is a $10 h^{-1} \mathrm{Mpc}$ co-moving box. We apply a zooming technique (Klypin et al. 2001) to select a Lagrangian volume of three viral radii centered in a Milky Way (MW)-size halo at redshift $z=0$. The volume is then re-simulated from $z=50$ with higher resolution and hydrodynamics. The high-resolution region has a radius of about $1.5 h^{-1}$ co-moving Mpc, and has about $4 \times 10^{5}$ dark matter particles with $5 \times 10^{6} M_{\odot}$ per particle. The volume is resolved with about $1.3 \times 10^{7}$ hydrodynamic cells with different levels of resolution.

The combined effect of stellar winds and supernova explosions at the resolved scale (Ceverino \& Klypin 2009) prevent the overcooling problem of galaxy formation at high redshifts (White \& Frenk 1991) and reduce the angular momentum problem found in early simulations (Navarro \& Steinmetz 2000). This results in models with flat rotation curves consistent with observations (Ceverino \& Klypin 2009), and is achieved without typical ad hoc assumptions about the physics at sub-resolution scales. These high-resolution simulations allow us to resolve the regime in which stellar feedback overcomes the radiative cooling. By resolving this regime, simulations naturally produce galactic scale outflows in star-forming galaxies (D. Ceverino \& A. Klypin 2010, in preparation) and galaxy formation proceeds in a more realistic, although violent way, through a combination of cold flows accretion, mergers, and galaxy outflows.

\subsection{Simulated Spectra}

To study the Mg II absorption arising in the gas halos within the simulations, we employ the following methods. For a given gas cell probed by a line of sight through the simulation box, the total hydrogen density, temperature, and metallicity are used to obtain the Mg II ionization fraction assuming photoionization conditions. Post simulation, we use Cloudy (V96b4; Ferland et al. 1998) with the Haardt \& Madau (1996) UV background spectrum at the appropriate redshift. The line of sight, the redshift, Mg II column density, and Doppler $b$ parameter (assuming thermal broadening) are computed for each cell.

Absorption spectra with the instrumental and noise characteristics of the HIRES spectrograph are generated assuming each cell gives rise to a Voigt profile at its line-of-sight redshift. We give each spectrum a signal-to-noise ratio of 50 per pixel, which corresponds to a limiting equivalent width detection of $0.005 \AA$ for unresolved lines. The spectrum for each sight line is then objectively analyzed for detectable absorption above the equivalent width threshold of $0.02 \AA$, which corresponds to $\log N\left(\mathrm{Mg}_{\mathrm{II}}\right)=11.7 \mathrm{~cm}^{-2}$ for $b=5 \mathrm{~km} \mathrm{~s}^{-1}$. The mean optical depths (mean redshifts), rest-frame equivalent widths and velocity widths, and other quantities are then measured (see Churchill \& Vogt 2001). The velocity zero point of the simulated absorption lines is set to the line-of-sight velocity of the simulated galaxy (center of mass of the stars).

To examine the three-dimensional spatial and kinematic properties of gas giving rise to $\mathrm{Mg}$ II absorption, we identify Mg II "absorbing gas cells" along each sight line as those which contribute to detected absorption in the simulated spectra; they are defined as cells that align within the range of line-of-sight velocities of the absorption. We account for multiple kinematics subsystems (Churchill \& Vogt 2001), regions of absorption separated by continuum. 


\subsection{Discussion of Simulated Galaxy Observations}

We focus on a single typical galaxy at $z=0.923$. The galaxy star formation rate is $\mathrm{SFR}=3.5 M_{\odot} \mathrm{yr}^{-1}$. The galaxy has a maximum rotation velocity of $180 \mathrm{~km} \mathrm{~s}^{-1}$ when observed edgeon. Based upon the Tully-Fisher relation, we derive a luminosity of $L_{B}=0.4 L_{B}^{*}$. The average luminosity for our sample is $\left\langle L_{B}\right\rangle=0.58 L_{B}^{*}$ excluding the $8 L_{B}^{*}$ galaxy. Thus, this simulated galaxy is well representative of our observational sample.

The simulated galaxy is probed with a square grid of sight lines at intervals of $7.5 \mathrm{kpc}$ that span $-110 \mathrm{kpc}$ to $+110 \mathrm{kpc}$ on the "sky" and $14 \mathrm{Mpc}$ along the line of sight. ${ }^{8}$ There are 900 total sight lines. The highest resolution of the adaptive mesh at $z=0.923$ is $225 \mathrm{pc}$. The gas contributing to detectable $\mathrm{Mg}$ II absorption is found in a range of cell resolutions from 225 to 1815 pc with the majority of the gas arising in cells of resolution $905 \mathrm{pc}$. We examine simulated quasar lines of sight for this galaxy with three different inclinations, $i=5^{\circ}$ (face-on), $45^{\circ}$, and $85^{\circ}$ (edge-on).

In Figure 9, we show the integrated total hydrogen column density, $N(\mathrm{H})$, over a $220 \mathrm{kpc}$ cube for the edge-on view of the galaxy. There is no absorption detected along the line of sight outside of this cube. The galaxy is clearly not in isolation. In the image, filaments and tidal stream material can be seen. Several low-mass satellites galaxies are also in the process of interacting with the main galaxy. We have superimposed squares over the sight lines where $\mathrm{Mg}$ II absorption was detected in simulated quasar spectra. Increasing square sizes indicate the absorption strength in four bins: $0.02 \leqslant W_{r}(2796) \leqslant 0.3$, $0.3<W_{r}(2796) \leqslant 0.6,0.6<W_{r}(2796) \leqslant 1.0$, and $1.0<$ $W_{r}(2796) \leqslant 3.0$. Out of the 900 lines of sight, $\mathrm{Mg}$ II absorption was detected in 87 for the edge-on case. Note that the absorption is not distributed ubiquitously on the sky around the galaxy, but traces the various structures around the galaxy. The covering fraction of the $\mathrm{Mg}$ II gas is low $(\sim 10 \%)$.

In Figure 10, we show the integrated neutral hydrogen column density, $N(\mathrm{HI})$, over the same cube. The $N(\mathrm{HI})$ range from roughly $10^{15}$ to $10^{22} \mathrm{~cm}^{-2}$. The structures that are associated with absorption stand out more in $\mathrm{HI}$. We see as expected (Churchill et al. 1999) that $\mathrm{Mg}$ II absorption is detected in regions with $N(\mathrm{HI}) \gtrsim 10^{16.5} \mathrm{~cm}^{-2}$. Regions in which $N\left(\mathrm{HI}_{\mathrm{I}}\right)>$ $10^{16.5} \mathrm{~cm}^{-2}$ that fall between the line-of-sight grid sampling are also expected to produced $\mathrm{Mg}$ II absorption. Note that as one goes to lower $\mathrm{N}(\mathrm{HI})$, the gas covering fraction increases.

In Figures 11 and 12, we present $N(\mathrm{H})$ and $N(\mathrm{HI})$, respectively, for the face-on view of the galaxy. Out of the 900 lines of sight, $\mathrm{Mg}$ II absorption was detected in 96. The covering fraction remains roughly $10 \%$. Again, the absorption primarily arises in streams and filaments.

We do not show the 45 degree view of the galaxy here. Out of the 900 lines of sight, $\mathrm{Mg}$ II absorption was detected in 124, and the covering fraction is roughly $20 \%$.

Overall, there are many complicated structures that reside within what we classically call a halo. Note that we find pockets of DLA H I column densities $\left[N(\mathrm{HI})>10^{20.3} \mathrm{~cm}^{-2}\right]$ out to $\sim 100 \mathrm{kpc}$. Although, the covering fractions of these dense regions are low, their presence suggests that it is possible to observe DLA absorption at high impact parameters. These large impact parameter DLA systems arise from the inner regions of satellite galaxies. These satellites have a luminosity range $0.003 L^{*} \leqslant L_{B} \leqslant 0.006 L^{*}$. Assuming standard $K$-corrections,

\footnotetext{
8 For this and all subsequent discussions, spatial quantities are quoted as proper lengths.
}

their apparent magnitude in the $H S T F 702 W$ filter range between $28 \lesssim m_{F 702 W} \lesssim 29$. These DLA producing satellites are well below the typical detectability of WFPC-2/HST and ACS/HST. Also note that the satellite in the upper right corner of Figure 12 has an $\mathrm{H}$ I morphology similar to galaxies seen on their first pass through a cluster, where gas is being stripped (e.g., Chung et al. 2007).

Focusing on the two small satellite galaxies and tidal stream in a $45 \times 45 \mathrm{kpc}$ region of the lower left quadrant of Figure 10, we present an example of simulated $\mathrm{Mg}$ II $\lambda 2796$ absorption spectra in Figure 13. The velocity zero point of the simulated spectra is set to the galaxy systemic velocity. The absorption profiles are quite similar to those detected in Mg II surveys. The simulated profiles have velocity spreads of $\sim 25-100 \mathrm{~km} \mathrm{~s}^{-1}$, some comprising multiple kinematic components and complex structures. The Mg II absorption spans across the galaxy systemic velocity or resides blueward of the galaxy systemic. Absorption arising near the galaxy systemic velocity or entirely to one side is consistent with the majority of our observational data.

In Figure 14, we show the Mg II $\lambda 2796$ absorption spectra over the $37.5 \times 45 \mathrm{kpc}$ central region of the edge-on galaxy shown in Figure 10. Individual absorption profile velocities, in the inner regions of the galaxy, show a variety of complex kinematics and optical depths. In our observational sample of galaxies, we have no galaxy-absorber systems that have impact parameters less than $25 \mathrm{kpc}$. In the simulations, the inner $\sim 15 \mathrm{kpc}$ contains some $\sim 200 \mathrm{~km} \mathrm{~s}^{-1}$ outflowing gas. The outflows are not strong, but their signatures are reflected by the complex kinematics of the absorption profiles in the inner regions. The reason why there is a line of sight near the center of the galaxy that does not produce $\mathrm{Mg}$ II absorption is because it is dominated by $10^{7} \mathrm{~K}$ gas. The profiles reduce in kinematic complexity rapidly as impact parameter increases.

To the degree that the simulations reflect the reality of the gaseous environments around galaxies, we find that $\mathrm{Mg}$ II absorption arises in many types of structures (metal enriched filaments, minor satellites, tidal streams, and within the region of the galaxy itself). As inferred from our simulated absorption-line survey, and guided by analysis of the three-dimensional simulations (examples provided by Figures 9-14), the simulations are not suggestive of $\mathrm{Mg}$ II absorption arising from spherical or disk-like halos with uniform gas covering fraction. We revisit the spatial and kinematic distribution of the absorbing gas in Section 5.5.

\subsection{Disk Halo Models of the Simulated Galaxy}

Given the structures shown in Figures 9-12, it would seem to be unrealistic to treat the halo as a monolithic thick disk. However, we have modeled all the simulated $\mathrm{Mg}$ II absorption profiles for each of the three galaxy inclinations with the disk halo model (Section 4).

We find that for the edge-on view, out of 87 lines of sight with detected $\mathrm{Mg}$ II absorption, $45 \%$ have kinematics consistent with the model (the full range of velocities can be explained). For $i=45^{\circ}$, out of 124 lines of sight, 26\% have kinematics consistent with the model. For the face-on view, out of 96 lines of sight, only one is consistent with the model. This is not surprising, since the projected maximum rotation velocity is small compared to the absorption spreads.

The conclusion to be drawn here is, that even if the halo gas does not rotate as a monolithic disk in the simulations, $\mathrm{Mg}_{\text {II }}$ absorption detected along some lines of sight can still appear to be consistent with a disk halo model. 
口
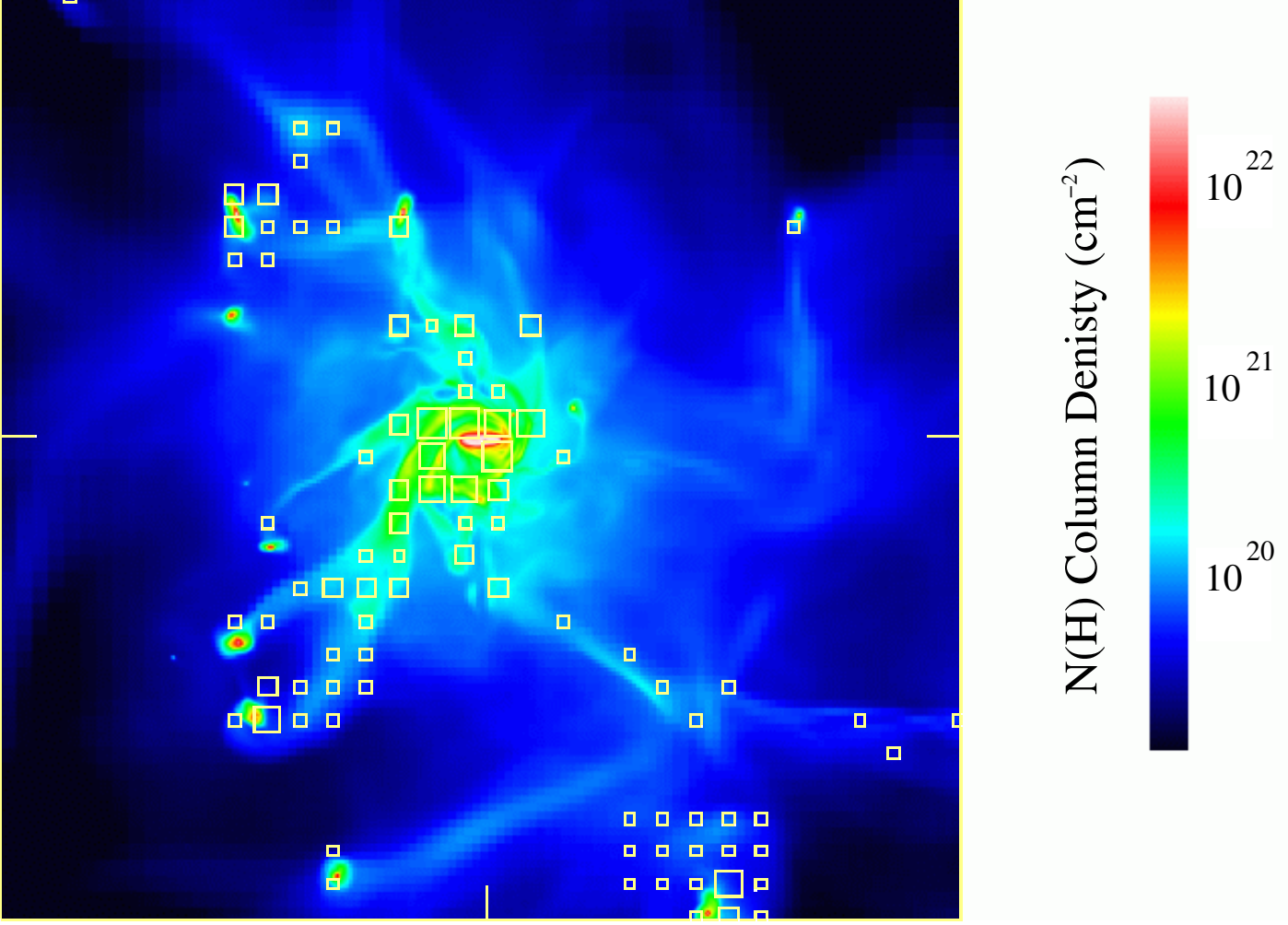

Figure 9. Integrated total hydrogen column density, $N(\mathrm{H})$, over a $220 \mathrm{kpc}$ cube is shown for a $z=0.923$ simulated galaxy viewed edge-on. The direction of the simulated quasar lines of sight are perpendicular to the plane of the image. Squares of increasing size are plotted where Mg II absorption was detected along the line of sight in the simulated quasar spectra. We apply an equivalent width detection limit of $W_{r}(2796) \geqslant 0.02 \AA$. The four square sizes indicate, in increasing order, Mg II absorption equivalent width bins of $0.02 \leqslant W_{r}(2796) \leqslant 0.3,0.3<W_{r}(2796) \leqslant 0.6,0.6<W_{r}(2796) \leqslant 1.0$, and $1.0<W_{r}(2796) \leqslant 3.0$, respectively.

(A color version of this figure is available in the online journal.)
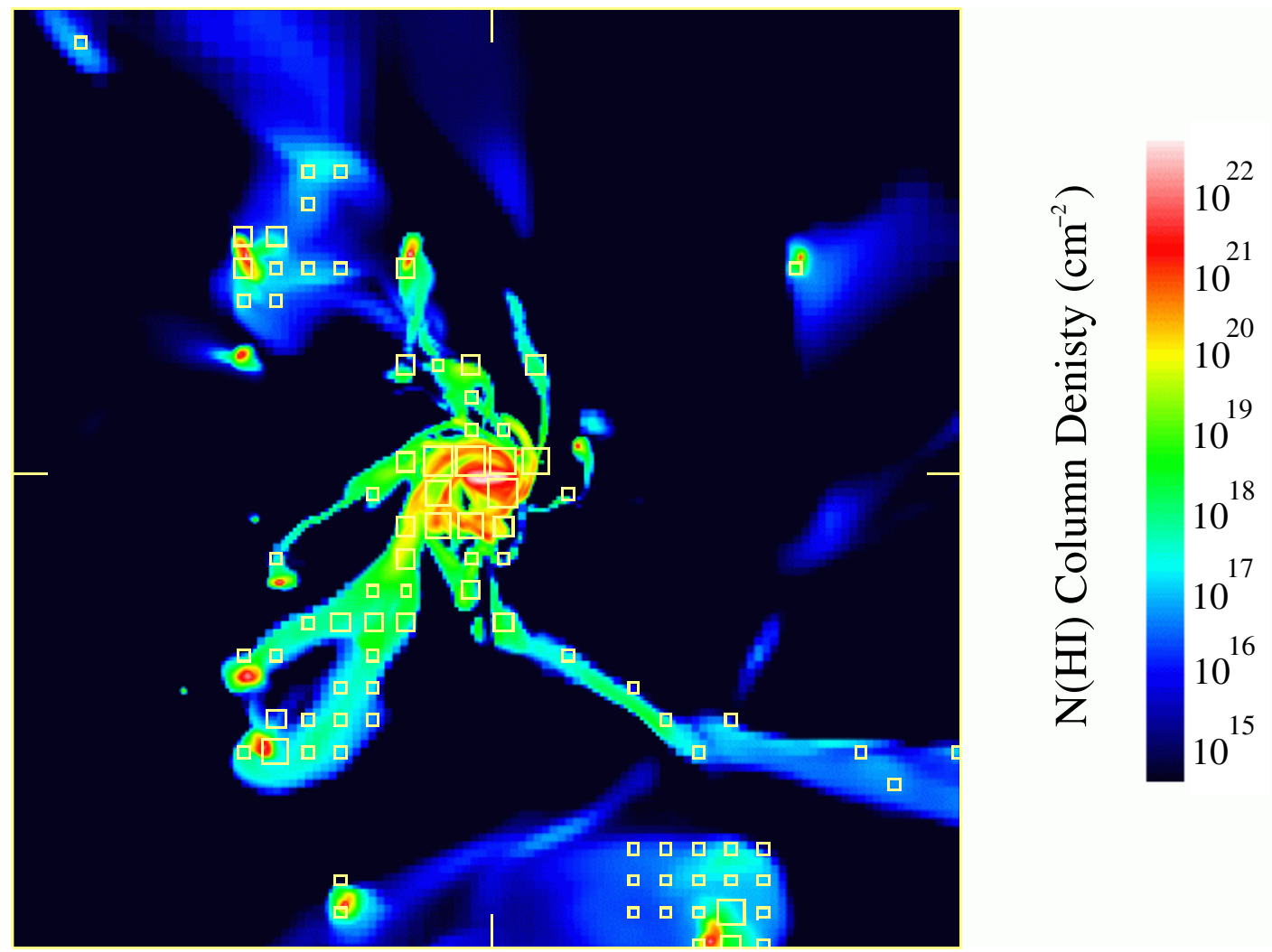

Figure 10. Same as Figure 9, except the integrated neutral $\mathrm{H}$ I column density, $N(\mathrm{HI})$, is shown. The direction of the simulated quasar lines of sight are perpendicular to the plane of the image. Note that small DLA regions, having $N\left(\mathrm{H}_{\mathrm{I}}\right) \geqslant 10^{20.3} \mathrm{~cm}^{-2}$, are seen beyond $\sim 85 \mathrm{kpc}$.

(A color version of this figure is available in the online journal.) 


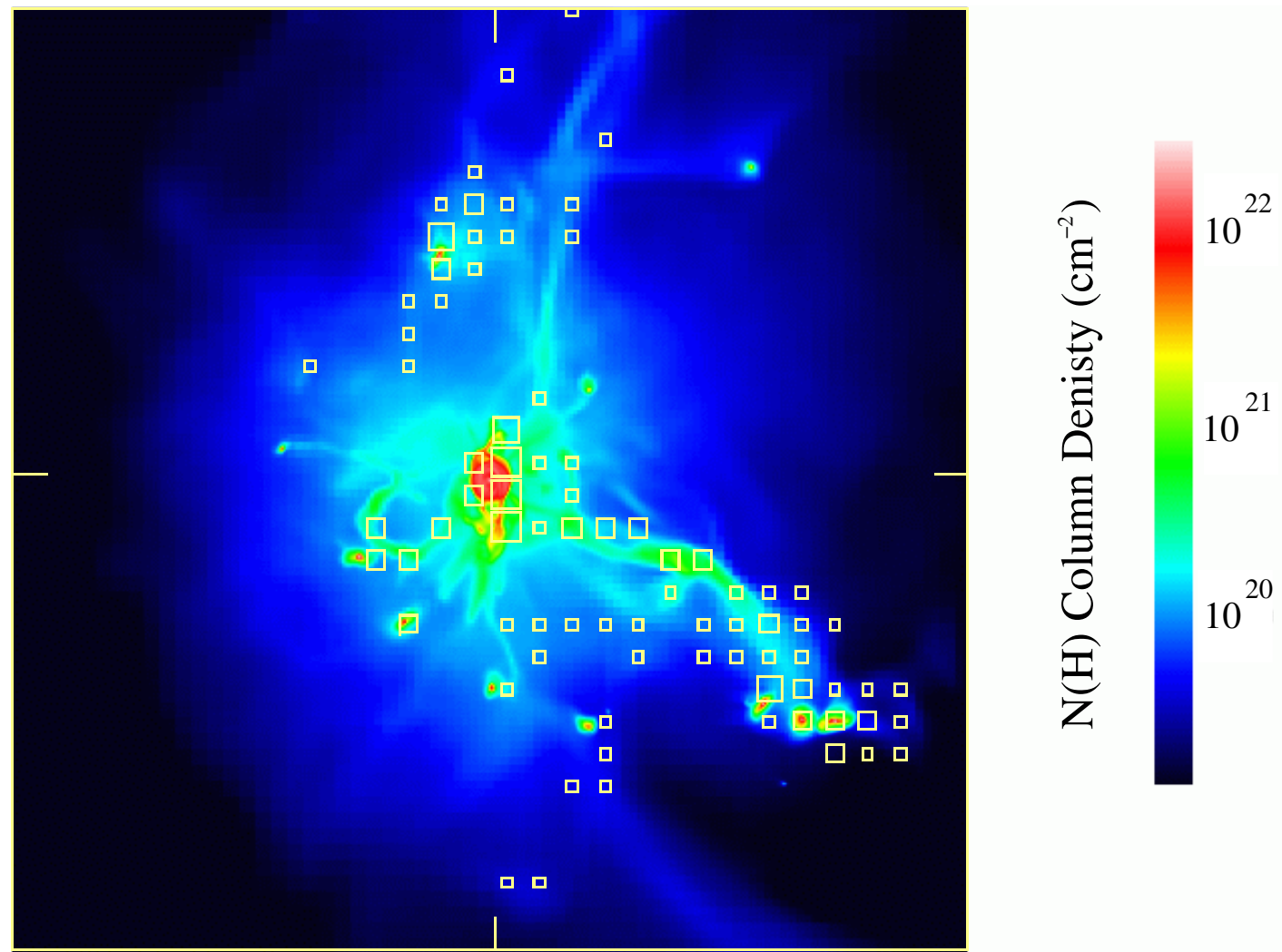

Figure 11. Same as Figure 9 for the same galaxy, except viewed face-on. The directions of the simulated quasar lines of sight are perpendicular to the plane of the image.

(A color version of this figure is available in the online journal.)
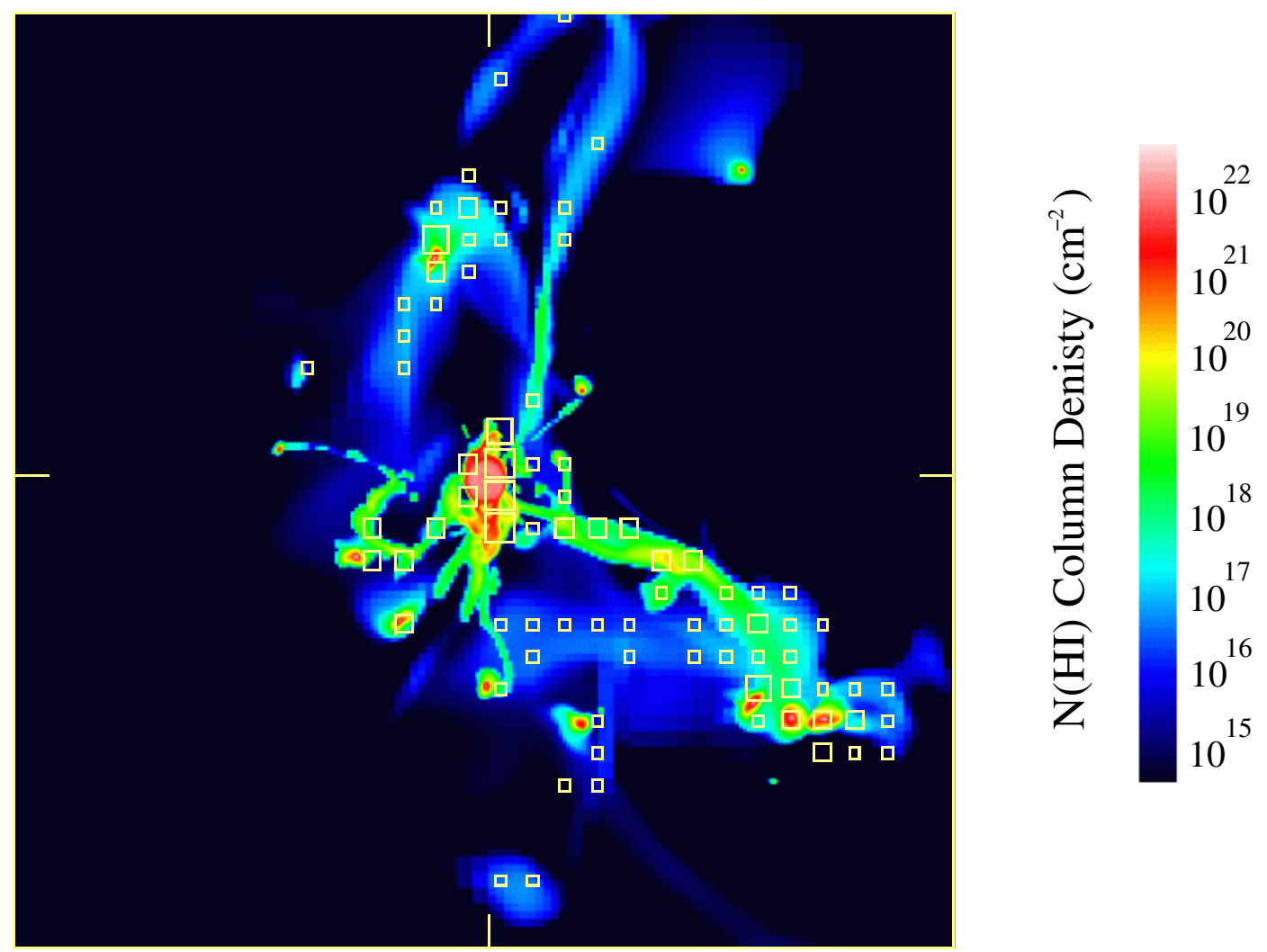

Figure 12. Same as Figure 10 for the same galaxy, except viewed face-on. The directions of the simulated quasar lines of sight are perpendicular to the plane of the image.

(A color version of this figure is available in the online journal.) 


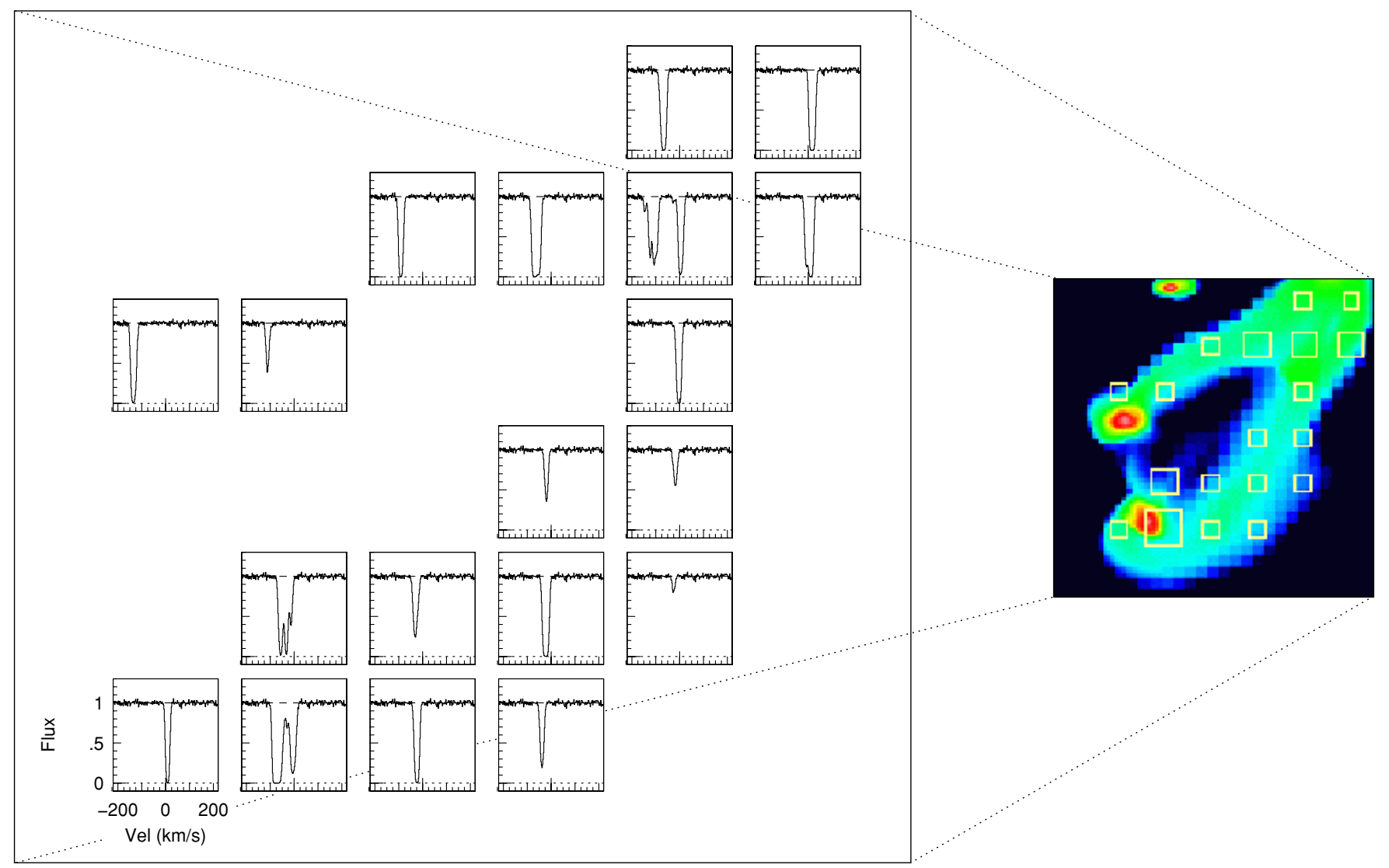

Figure 13. $45 \times 45 \mathrm{kpc}$ region extracted from the lower left quadrant of Figure 10. The region shows two satellite galaxies and their tidal streams. The velocity zero point of the absorption profiles is the galaxy systemic velocity. We enforced a detection sensitivity limit of $W_{r}(2796) \geqslant 0.02 \AA$. The majority of the Mg II absorption arising in this tidal stream is radially infalling toward the galaxy.

(A color version of this figure is available in the online journal.)

\subsection{Halo Gas Spatial and Velocity Distribution}

To understand what kinematic mechanisms are responsible for the $\mathrm{Mg}$ II absorption velocity spreads measured in the simulated spectra, we examine the velocities and spatial distributions Mg II absorbing gas.

In Figure 15, we present the three-dimensional spatial distribution of $N(\mathrm{Mg}$ II $)>10^{11.5} \mathrm{~cm}^{-2}$ gas contributing to the Mg II absorption along the lines of sight. The edge-on galaxy is located at the origin and the lines of sight are parallel to the $x$-axis. Gas with $N(\mathrm{Mg}$ II $)<10^{11.5} \mathrm{~cm}^{-2}$ is not shown for clarity (which corresponds to an equivalent width limit of $0.012 \AA$ for $b=5 \mathrm{~km} \mathrm{~s}^{-1}$ ). From this view, the tidal streams and filaments can be visually discerned. There are also isolated clouds that produce $\mathrm{Mg}$ II absorption. Regions that contribute to absorption have physical sizes of $225 \mathrm{pc}$ (simulation resolution) to $\sim 20 \mathrm{kpc}$. All the absorption along the lines of sight occurs within $\pm 110 \mathrm{kpc}$ from the center of the galaxy.

The gas is color coded as a function of line-of-sight velocity relative to the galaxy systemic velocity. Gas that is colored red (blue) is moving away from (toward) the observer. The gas has line-of-sight velocities ranging from $-160 \mathrm{~km} \mathrm{~s}^{-1} \leqslant v_{\text {los }} \leqslant$ $+160 \mathrm{~km} \mathrm{~s}^{-1}$. The line-of-sight velocity dispersion in the inner regions near the galaxy appears inconsistent with disk rotation. The somewhat randomized velocities reflect the winds. Upon carefully examining the gas velocities along a particular lineof-sight, velocity gradients can be observed. For example, the region seen in Figure 15 at $x=100, y=-50 \mathrm{kpc}$, has velocity gradients of $\sim 30-40 \mathrm{~km} \mathrm{~s}^{-1}$.
In Figure 16, we present the same three dimension spatial distribution shown in Figure 15, except that the gas is color coded as a function of radial velocity relative to the galaxy. Gas colored red (blue) has a radial velocity component that is outflowing from (infalling toward) the galaxy. This combined spatial and kinematic representation provides an holistic view of the halo dynamics. The gas has radial velocities ranging from $-200 \mathrm{~km} \mathrm{~s}^{-1} \leqslant v_{r} \leqslant+100 \mathrm{~km} \mathrm{~s}^{-1}$. If one focuses on the filament structure $(x=0 \mathrm{kpc}, y=0-100 \mathrm{kpc})$ in the plane of the galaxy, one can see the strong velocity gradient. The gas along the filament far from the galaxy increases in infall velocity from $\sim 70$ to $\sim 200 \mathrm{~km} \mathrm{~s}^{-1}$ as it approaches the galaxy center. The same dynamics can be seen along the tidal stream $(x=0-50 \mathrm{kpc}, y=-50 \mathrm{kpc})$ originating from the two small satellite galaxies.

In Figure 17(a), we show the probability distribution of the total gas velocity of the $\mathrm{Mg}$ II absorbing gas cells. What we call the probability distribution is the area normalized frequency distribution that we detected in the simulations. The total velocity is the magnitude of the gas velocity vector relative to the galaxy. Since our observational data have only impact parameters greater than $\sim 20 \mathrm{kpc}$, we exclude all absorbing gas within $D \leqslant 20 \mathrm{kpc}$ of the simulated galaxy. The velocities range from $50 \mathrm{~km} \mathrm{~s}^{-1} \lesssim v_{\text {tot }} \lesssim 250 \mathrm{~km} \mathrm{~s}^{-1}$ with a peak at $185 \mathrm{~km} \mathrm{~s}^{-1}$ and secondary maxima at $100 \mathrm{~km} \mathrm{~s}^{-1}$. In Figure 17(b), we show the probability distribution of radial velocities of the $\mathrm{Mg}$ II absorbing cells. The velocities range from $-200 \mathrm{~km} \mathrm{~s}^{-1} \lesssim v_{r} \lesssim+110 \mathrm{~km} \mathrm{~s}^{-1}$ with a maximum at $-160 \mathrm{~km} \mathrm{~s}^{-1}$. It appears that, beyond $D \geqslant 20 \mathrm{kpc}$, most of the 


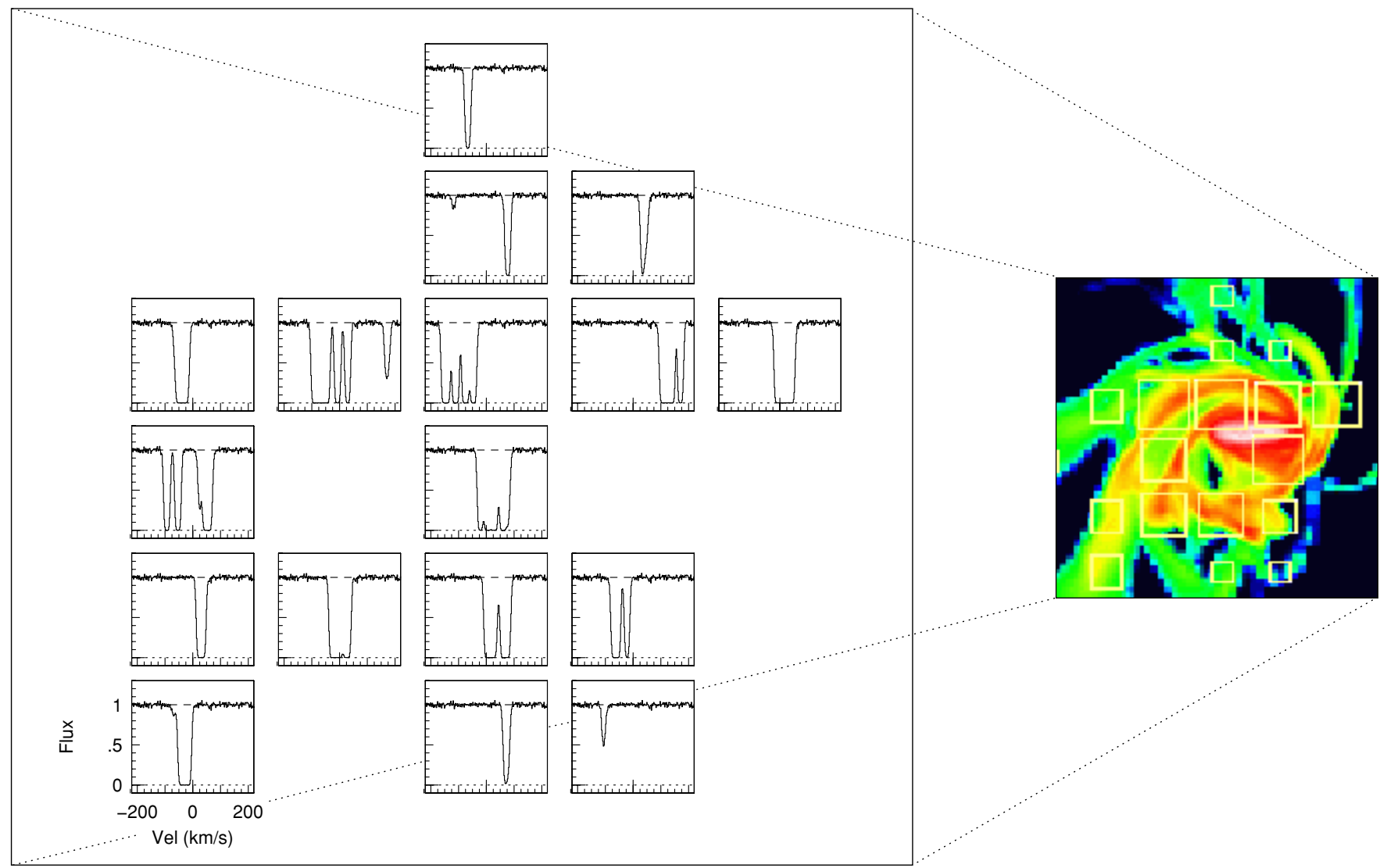

Figure 14. $37.5 \times 45 \mathrm{kpc}$ inner central region of the edge-on galaxy seen in Figure 10. The velocity zero point of the absorption profiles is the galaxy systemic velocity. We enforced a detection sensitivity limit of $W_{r}(2796) \geqslant 0.02 \AA$. In the inner $\sim 15 \mathrm{kpc}$ of the galaxy center, we find some gas outflowing at $\sim 200 \mathrm{~km} \mathrm{~s}^{-1}$. The Mg II absorption profiles produced by these outflows are similar to those seen for Q0002+051 G1 (Figure 2(b)) and Q0836+113 G1 (Figure 4(b)).

(A color version of this figure is available in the online journal.)

gas is infalling toward the galaxy and very little is outflowing. In Figure 17(c), we show the ratio of the radial to the total velocity. The bulk of the gas is dominated by radial infalling velocities.

Drawing from the number of lines of sight we have through the simulations, we produced the probability distribution of absorption velocity offsets from the galaxy systemic velocity $\left(\Delta v=v_{\text {abs }}-v_{\text {gal }} \mathrm{km} \mathrm{s}^{-1}\right)$ using the simulated absorption profiles. The quantity $v_{\text {abs }}$ is obtained by calculating the optical depth weighted mean of the profiles (the velocity at which there is equal optical depth to both sides along the profiles). In Figures 18(a), (b), and (c), we show the velocity offset probability distributions for the edge-on, $i=45^{\circ}$, and face-on orientations, respectively. For the edge-on case, the absorption velocity offset ranges from $\pm 100 \mathrm{~km} \mathrm{~s}^{-1}$ with a strong peak around $+30 \mathrm{~km} \mathrm{~s}^{-1}$; it is highly probable to detect absorption to one side of the galaxy systemic velocity. For the $i=45^{\circ}$ case, the velocity spread increases to about $\pm 200 \mathrm{~km} \mathrm{~s}^{-1}$ and develops multiple peaks at $\pm 150 \mathrm{~km} \mathrm{~s}^{-1}$ and at $\sim+50 \mathrm{~km} \mathrm{~s}^{-1}$. The probability of detecting gas at the galaxy systemic velocity is significantly smaller than over the range $|50-150| \mathrm{km} \mathrm{s}^{-1}$. For the face-on case, the velocity dispersion is still around $\pm 200 \mathrm{~km} \mathrm{~s}^{-1}$. Again, it is unlikely that absorption will be detected at the galaxy systemic velocity.

Since most of the $\mathrm{Mg}$ II gas arises between $\pm 200 \mathrm{~km} \mathrm{~s}^{-1}$, with very little at the galaxy systemic velocity, and since most projected galaxy rotation curves have maximum velocities of $\pm 200 \mathrm{~km} \mathrm{~s}^{-1}$, it may not be a surprise to observe $\mathrm{Mg}$ II absorption aligned with the observed galaxy rotation curve.
These results are consistent with the findings of Bouché et al. (2007), who detected galaxy $\mathrm{H} \alpha$ emission within $\pm 200 \mathrm{~km} \mathrm{~s}^{-1}$ of the optical depth weighted mean $\mathrm{Mg}$ II absorption redshift.

\subsection{Shortcoming of the Simulations}

The technique of quasar absorption lines through cosmological simulations is one of several promising approaches to understanding the dynamics of galaxy halos. At the present time, simulations of galaxy formation in the cosmological context still need to achieve greater accuracy for modeling stellar feedback. For this study, we employed a feedback recipe that successfully results in extended metal enriched gas around galaxies. These simulations result in an equivalent width distribution with an under abundance of larger equivalent widths and a relative over abundance of smaller equivalent widths. They also under predict the observed $\mathrm{Mg}$ II mean absorption covering fraction range of 0.2-0.9 (Tripp \& Bowen 2005; Chen \& Tinker 2008; Kacprzak et al. 2008; Barton \& Cooke 2009). The covering fractions for all absorption above $0.02 \AA$ are as follows: (1) $i=90^{\circ}$ (edgeon): total $10 \%$, weak $6 \%$, strong $3 \%$; (2) $i=0^{\circ}$ (face-on): total $10 \%$, weak $6 \%$, strong $3 \%$; and (3) $i=45^{\circ}$ : total $14 \%$, weak $10 \%$, strong $5 \%$.

These mismatches with observations could either be a result of the method in which $\mathrm{Mg}$ II column densities are determined in the simulations or observational biases. In the simulations, the determination of the $\mathrm{Mg}$ II ionization fraction may be underestimated due to the fact that we do not account for 


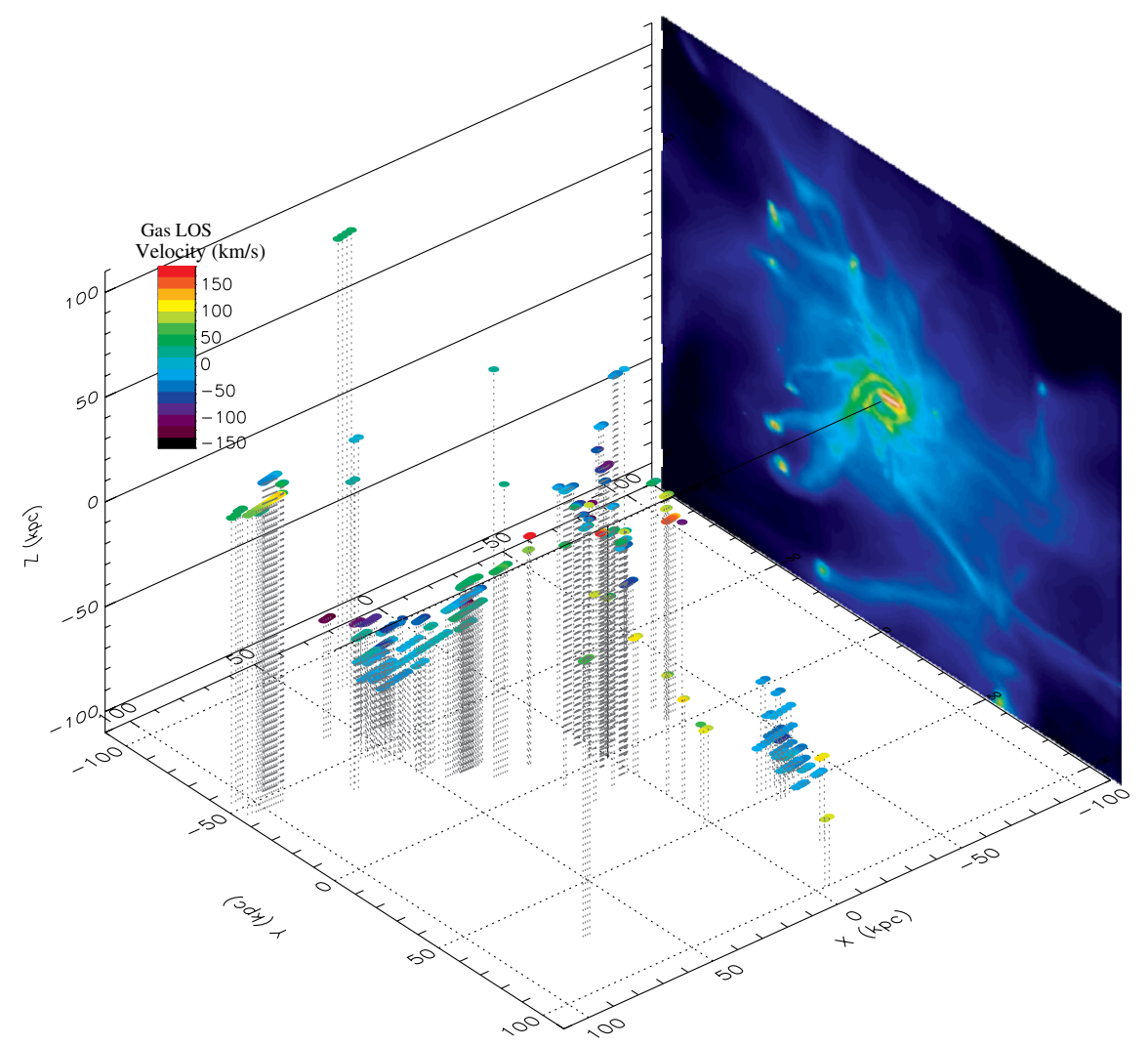

Figure 15. Spatial distribution of $N(\mathrm{Mg}$ II $)<11.5 \mathrm{~cm}^{-2}$ gas contributing to the $\mathrm{Mg}$ II absorption along the lines of sight. The edge-on galaxy is located at the origin; the black horizontal line represents a line of sight passing through the galaxy center. The observer is looking along the positive $x$-direction. All simulated lines of sight are parallel to the $x$-axis. The absorbing gas is color coded as a function of line-of-sight velocity relative to the galaxy systemic velocity, as coded in the legend. Red absorbing gas is moving away from the observer; blue is moving toward.

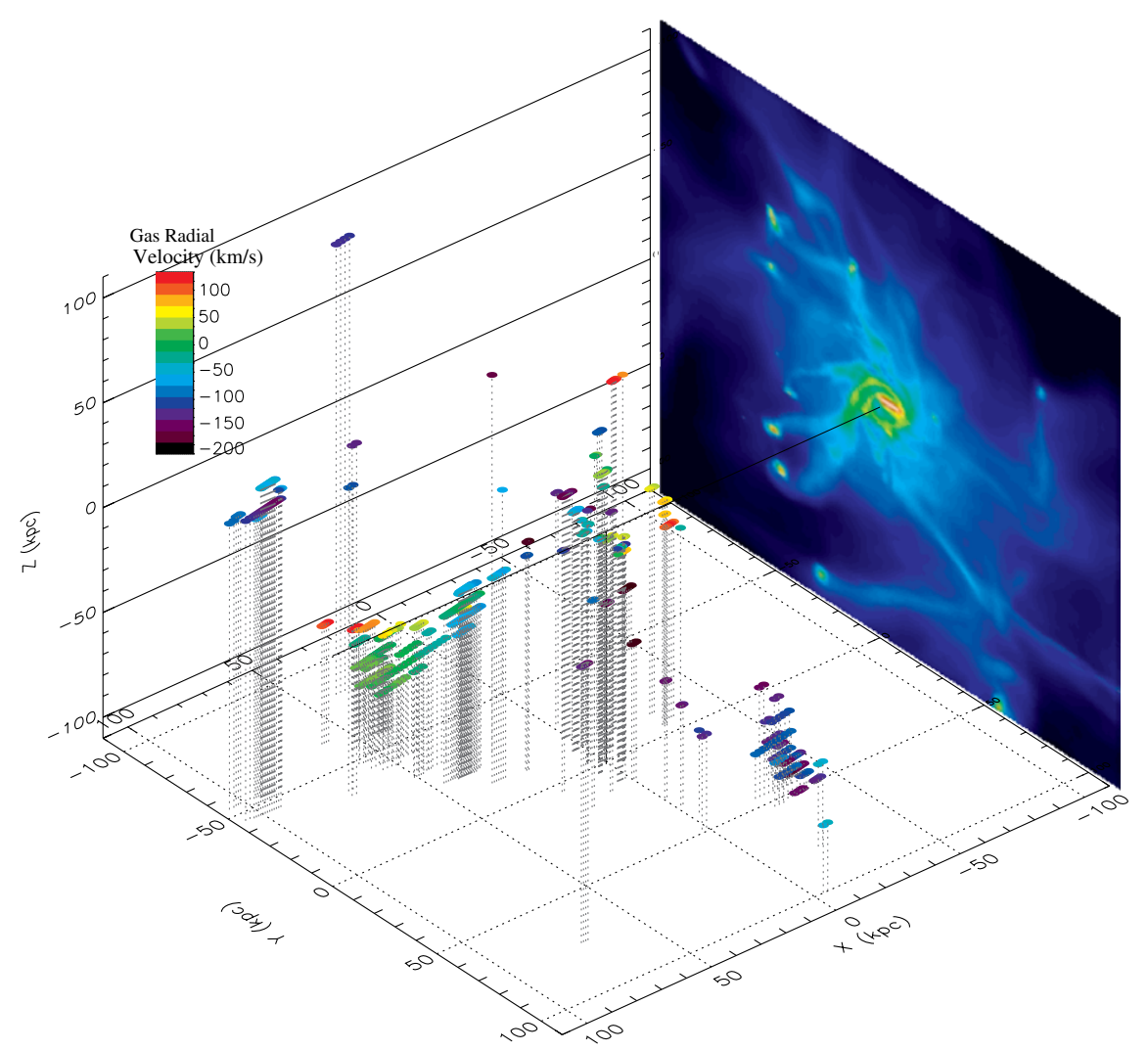

Figure 16. Same as Figure 15, except the velocity color coding is for the radial velocity component with respect to the galaxy. Red absorbing gas is outflow; blue is inflow. The majority of the $\mathrm{Mg}$ II absorption arises in filaments and tidal streams and is infalling toward the galaxy. 

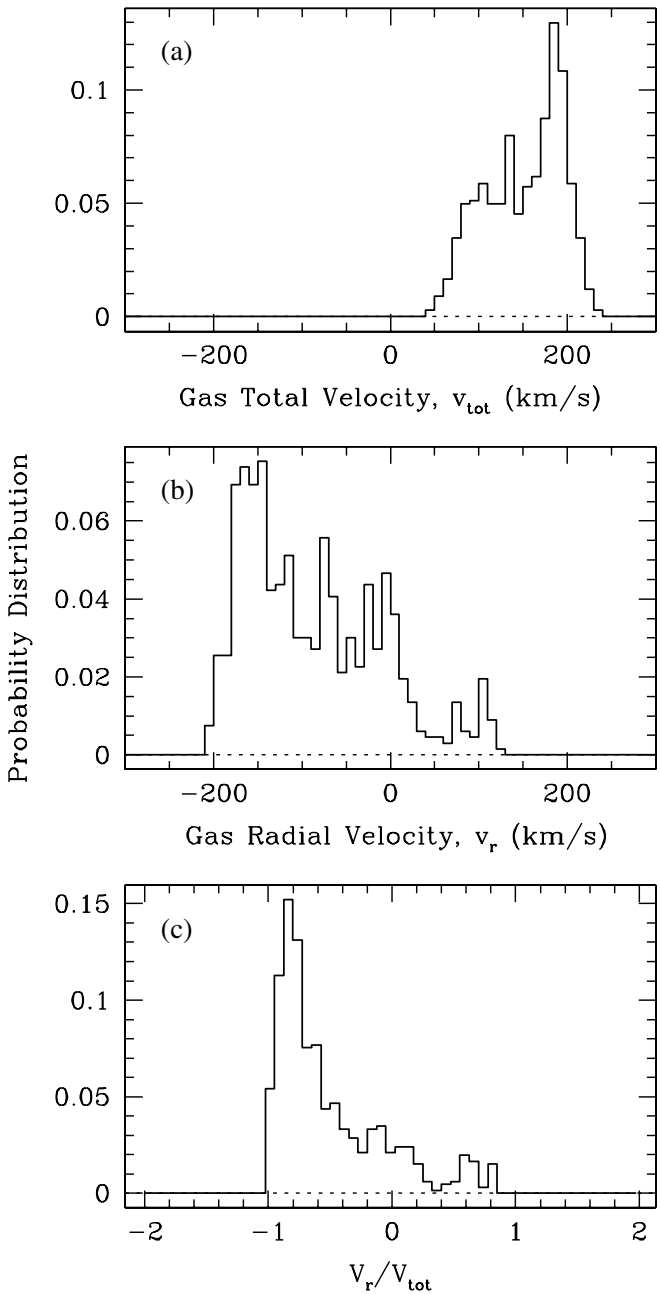

Figure 17. Velocity distributions of $\mathrm{Mg}$ II absorbing gas cells within the $220 \mathrm{kpc}$ cube of the simulation shown in Figures 15 and 16. Gas within a $20 \mathrm{kpc}$ radius of the galaxy is omitted. (a) The total velocity distribution. (b) The radial velocity distribution. (c) The distribution of the ratio of radial to total velocity. A ratio of $v_{r} / v_{\text {tot }}= \pm 1$ indicates pure radial motion.

shielding of UV photons in the ionization corrections. It is also possible that the resolution of the simulations may influence the derived $\mathrm{Mg}$ II column densities and that higher resolution may in fact lead to higher column densities. We aim to analyze such issues in future work. A possible observational biases that galaxies are selected, identified, and assigned to already known absorption systems. This may elevate the inferred covering fraction (e.g., Tripp \& Bowen 2005). We do emphasize, however, that in the simulations we do detect $\mathrm{Mg}$ II absorption out to $\sim 120 \mathrm{kpc}$, as seen in current observations (Churchill et al. 2005; Zibetti et al. 2007; Kacprzak et al. 2008).

As an additional caveat, we also remark that the experiment to examine the spatial and kinematic relationship between the galaxy and the $\mathrm{Mg}$ II absorbing structures in the simulations is very different than the observational experiment in one regard. We examine a grid of sight lines through a single simulated galaxy, which is in a unique environment and undergoes a unique evolution in the IGM. The observational data, on the other hand, sample a single line of sight through various galaxies in various environments and with various evolutionary histories and with random orientation through the galaxy and environment.

We re-emphasize that the simulations, as we have applied them here, provide a first view of the types if physical structures and their spatial and kinematic relationship to a galaxy in the

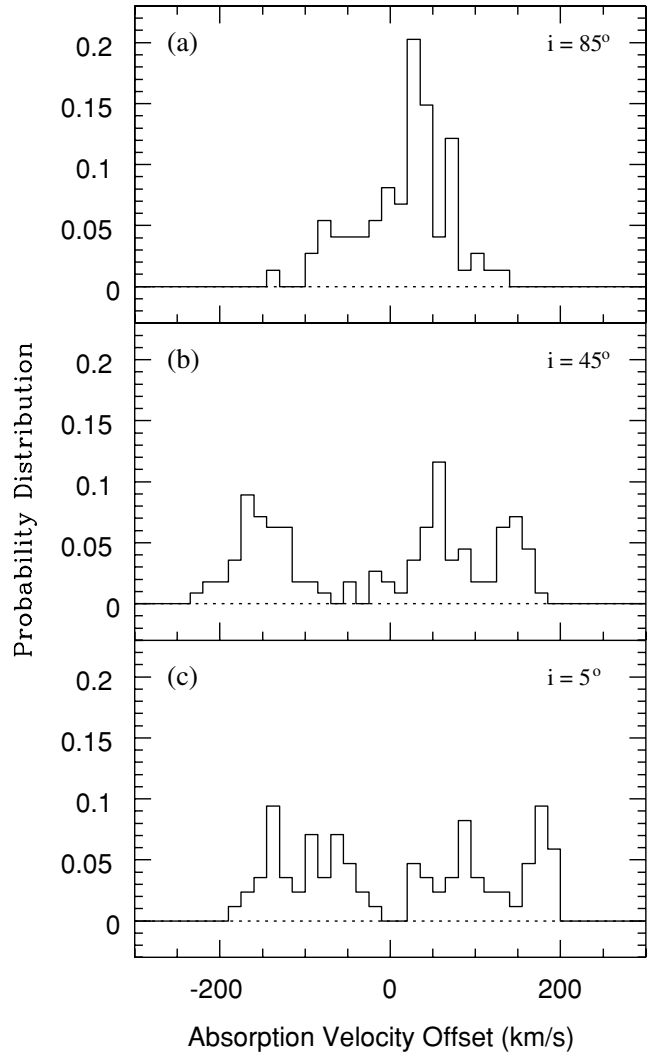

Figure 18. Probability distributions of the mean velocities of the simulated Mg II absorption profiles arising in the lines of sight, for three galaxy orientations. The quantity $v_{\mathrm{abs}}$ is the optical depth weighted mean of the profiles. Lines of sight with impact parameters less that $20 \mathrm{kpc}$ are omitted. (a) $i=85^{\circ}$, edge-on. (b) $i=45^{\circ}$. (c) $i=5^{\circ}$, face-on. The absorption profiles span the rotation velocities of the simulated galaxy $\left(v_{c}=180 \mathrm{~km} \mathrm{~s}^{-1}\right)$.

cosmological environment that give rise to $\mathrm{Mg}$ II absorption. Though the details of the $\mathrm{Mg}$ II absorbing properties are not yet tuned to observations (and we mention that our experiment is only for a single galaxy using an incomplete sampling of its halo- $\mathrm{a}$ full comparison with survey statistics is not entirely applicable), the dynamical structures themselves in the simulations are robust. That we find some parts of these structures give rise to $\mathrm{Mg}$ II absorption is not outside expectation given the densities and temperatures of the gas. The upshot is that, given these discrepancies and concerns, the simulations are not expected to provide a fully quantitative comparison with the data. However, the simulations do provide a fully self-consistent galaxy model from which we acquired valuable insights for interpreting $\mathrm{Mg}$ II absorption-line observations.

\subsection{Summary III: Galaxy Simulations and Halo Gas Distributions}

In our study of $z=0.923$ galaxy at three inclination angles with respect to the simulated quasar lines of sight, we have detected $\mathrm{Mg}$ II absorption in a variety of structures. Mg II absorption was detected in inflowing metal enriched filaments, tidal streams, small satellites, and gas around the host galaxy. The absorption resides in a "halo" of about $100 \mathrm{kpc}$ in size. The types of structures that form in the simulations (see Figures 9-12) are a challenge to models in which the absorption is assigned to thick disks (e.g., Charlton \& Churchill 1996) and symmetrically distributed halos (e.g., Mo \& MiraldaEscude 1996). The structures also may provide further guidance 
for expanding upon radial density dependent halo occupation models (e.g., Tinker \& Chen 2008). The spatial distribution of filaments, tidal streams, and satellite galaxies are asymmetric, patchy (low volume filling factor), complex, and part of a cosmological setting.

DLA H I column densities are seen out to $\sim 100 \mathrm{kpc}$, and arise in low-mass satellite galaxies. These galaxies are below the detection limits of even deep HST images. Although, the covering fractions of these dense regions are low, this might explain why some bright galaxies at DLA redshifts are found at large impact parameters (e.g., the 3C $336 z=0.656$ DLA at $D=100 \mathrm{kpc}$; Steidel et al. 1997).

In the simulations, the kinematics are closely coupled to the gas structures. Metal enriched tidal streams and filaments are dominated by infall to the central galaxy, and these structures are selected by $\mathrm{Mg}$ II absorption (see Figures 15 and 16). In fact, we find that gas giving rise to the $\mathrm{Mg}$ II absorption is dominated by inflow (see Figure 17). Inflow velocity gradients are apparent, such that the infall increases as the gas approaches the galaxy. This inflow is not spatially symmetric.

Similar to the observations, the velocity and spatial distributions from the simulations conspire to give rise to $\mathrm{Mg}$ II absorption to one side of the galaxy systemic velocity even though the absorbing gas is not rotating with the star-forming component (see Figures 13 and 14). The velocity offset probability distribution (relative to the simulated galaxy) spans $\sim 200 \mathrm{~km} \mathrm{~s}^{-1}$ with lowest probability of detecting Mg II at the galaxy systematic velocity (see Figure 18). Thus, the fact that we observe $\mathrm{Mg}$ II absorption velocities consistent with the galaxy rotation curves may be a natural consequence of the spatial and kinematic distributions of gas in complex environments surrounding galaxies.

The current feedback recipes used in the simulations successfully produces in extended metal enriched halo gas around galaxies. However, for this single galaxy we derive covering fractions that are lower than the current observed mean values of 0.2-0.8 (Tripp \& Bowen 2005; Chen \& Tinker 2008; Kacprzak et al. 2008; Barton \& Cooke 2009). In this particular galaxy halo, we also find an under abundance of larger equivalent widths and a relative over abundance of smaller equivalent widths. We have yet to determine if some of these mismatches are due to observational biases in current studies or issues with the feedback/baryon physics implemented in the simulations. However, the structures in the simulations still provide valuable insight for interpreting Mg II absorption-line observations.

\section{LARGE-SCALE GALACTIC WINDS: OBSERVATIONS AND SIMULATIONS}

In our analysis of the simulated galaxy, we do not find systematic rotation near the galaxy, as was suggested by Lanzetta \& Bowen (1992), but that the gas kinematics reflects processes such as winds and chimneys. These are characteristic of stellar feedback processed that successfully circumvent the over cooling and angular momentum problems that have plagued simulations (Ceverino \& Klypin 2009).

Observationally, Weiner et al. (2009) have inferred outflows in $\mathrm{Mg}$ II absorption associated with $z \sim 1$ star-forming galaxies $\left(10 M_{\odot} \mathrm{yr}^{-1} \lesssim \mathrm{SFR} \lesssim 40 M_{\odot} \mathrm{yr}^{-1}\right)$. The outflow velocities are proportional to $\mathrm{SFR}^{0.3}$. The SFR in our simulated galaxy is $3.5 M_{\odot} \mathrm{yr}^{-1}$. Calibrating to their observations, our galaxy is expected to have winds in the range $220-350 \mathrm{~km} \mathrm{~s}^{-1}$. Our simulated $\mathrm{Mg}$ II profiles indicated some outflowing gas with velocities of $\sim 200 \mathrm{~km} \mathrm{~s}^{-1}$ in the inner $15 \mathrm{kpc}$ (independent of galaxy disk inclination). The absorption profiles produced by the outflows are saturated and span both sides of the systemic velocity of the simulated galaxy (see Figure 14). Thus, the saturated Mg II absorption profile that spans both sides of the galaxy systemic velocity might be direct signature of outflows.

Two galaxies in our sample, Q0002+051 G1 and Q0836+113 G1, have saturated profiles that span both sides of the galaxy systemic velocity. These saturated, symmetric profiles are also observed at high redshift $(z \sim 2)$ and span the galaxy systemic velocity. The absorption is attributed to large-scale galactic outflows which can be detected at least out to impact parameters of $\sim 70 \mathrm{kpc}$ (C. C. Steidel et al. 2010, in preparation). The outflows are determined to be more or less symmetric and radial, giving rise to symmetric absorption profiles. These high-redshift galaxies do not exhibit substantial kinematic structure and are instead consistent with dispersion-dominated velocity fields around $80 \mathrm{~km} \mathrm{~s}^{-1}$ (Law et al. 2007), similar to the shear observed for Q0002+051 G1 and Q0836+113 G1. Given the similarities between these two galaxies and those at high redshift observed to have outflows, one possibility is that large-scale outflows are responsible for the absorption velocities associated with both galaxies. To explore the outflow scenario for Q0002+051 G1 and Q0836+113 G1, we examine the galaxy SFRs.

For Q0836+113 G1, we measure a [O II] line flux of $3.4 \pm$ $0.43 \times 10^{-16} \mathrm{erg} \mathrm{s}^{-1} \mathrm{~cm}^{-2}$. We use the relation from Kewley et al. (2004) to obtain a SFR of $6.5 \mathrm{M}_{\odot} \mathrm{yr}^{-1}$. For Q0002+051 G1, we determined the star formation from the UV flux at $1700 \AA$ which was derived from the $R$-band magnitude of the galaxy (Steidel et al. 1994). The robustness of this method has been demonstrated by Erb et al. (2006). From the measured UV flux of $2.3 \pm 0.5 \times 10^{-16} \mathrm{erg} \mathrm{s}^{-1} \mathrm{~cm}^{-2}$, we derive a SFR of $6.5 M_{\odot} \mathrm{yr}^{-1}$. The SFRs are not corrected for the internal extinction of the galaxies and are thus lower limits.

Using the results of Weiner et al. (2009), both galaxies are expected to have outflows with velocities in the rage $270-430 \mathrm{~km} \mathrm{~s}^{-1}$. Both galaxies are at impact parameters of $\sim 25 \mathrm{kpc}$, which is near the boundary of where we lose the signature of outflows for our simulated galaxy. Although Q0836+113 G1 and Q0002+051 G1 have Mg II absorption velocity widths of 290 and $470 \mathrm{~km} \mathrm{~s}^{-1}$, respectively, which are comparable to the outflow velocity range predicted from the results of Weiner et al. (2009).

Heckman $(2002,2003)$ discusses that the star formation per unit area is a more relevant indicator of galaxy outflows. These outflows are ubiquitous in galaxies where the global SFR per unit area exceeds $\Sigma=0.1 M_{\odot} \mathrm{yr}^{-1} \mathrm{kpc}^{-2}$, where the area is defined by the half light radius of the galaxy. This criterion applies to local starbursts and even high-redshift Lyman break galaxies. The ISM entrained in the winds have outflow speeds of $\sim 100$ to $\sim 1000 \mathrm{~km} \mathrm{~s}^{-1}$. For Q0002+051 G1, we obtain a $\Sigma \geqslant 0.35$ $M_{\odot} \mathrm{yr}^{-1} \mathrm{kpc}^{-2}$. For Q0836+113 G1, we obtain a $\Sigma \geqslant 0.14$ $M_{\odot} \mathrm{yr}^{-1} \mathrm{kpc}^{-2}$. Thus, both of the galaxies are expected to have outflow signatures.

We estimate that the simulated galaxy has a $\Sigma=0.08$ $M_{\odot} \mathrm{yr}^{-1} \mathrm{kpc}^{-2}$, which is slightly less that the criterion of Heckman. This is consistent with our outflow velocities derived from the $\mathrm{Mg}$ II absorption profiles since we do not see evidence of strong large-scale outflows. This particular simulated galaxy may not be well representative of the Q0002+051 G1 and Q0836+113 G1 galaxies.

In summary, galaxies Q0002+051 G1 and Q0836+113 G1 are kinematically similar to high-redshift absorption-selected galaxies. The SFRs and $\Sigma$ s for both galaxies exceed the limits 
where strong outflows are expected. Given the large impact parameters that outflows are detected at high redshift, it is quite possible the observed $\mathrm{Mg}$ II absorption kinematics for galaxies Q0002+051 G1 and Q0836+113 G1 are signatures of outflowing gas.

\section{CONCLUSIONS}

We have examined and compared the detailed galaxy and Mg II absorbing kinematics for a sample of 10 intermediate redshift galaxies. The galaxies have a wide range of inclinations and orientations with respect to the background quasar. The galaxy-quasar impact parameters range from $26 \mathrm{kpc} \leqslant D \leqslant$ $108 \mathrm{kpc}$. The galaxy rotation curves were obtained from ESI/ Keck spectra and the Mg II absorption profiles were obtained from HIRES/Keck and UVES/VLT quasar spectra. In an effort to compare the relative kinematics, we used a thick disk halo model to compute the expected absorption velocities through a monolithic gaseous halo.

To obtain theoretical insights into the gas dynamics and spatial distribution of halos, we used the technique of quasar absorption lines to analyze $\mathrm{Mg}$ II absorption around a galaxy in a high-resolution cosmological simulation of galaxy formation. The galaxy was probed with a square grid of sight lines at intervals of $7.5 \mathrm{kpc}$ that span $-110 \mathrm{kpc}$ to $+110 \mathrm{kpc}$ for a total of 900 sight lines. We examined this galaxy at three different inclinations, face-on, $45^{\circ}$, and edge-on.

Our mains results can be summarized as follows.

1. For all 10 galaxies, the velocity of the strongest $\mathrm{Mg}$ II absorption component lies in the range of the observed galaxy rotation curve. In seven of 10 cases, the $\mathrm{Mg}_{\text {II }}$ and $\mathrm{Mg}$ I absorption velocities reside fully to one side of the galaxy systemic velocity. The strongest absorption usually aligns with one arm of the rotation curve. In the three remaining cases, the absorption velocities span both sides of the galaxy systemic velocity. Two of those three (Q0002+051 G1 and Q0836+113 G1) have strong saturated absorption on both sides of the galaxy systemic velocity. The third (Q1127-145 G3) has two very weak clouds, and therefore probes low column density gas.

2. For galaxies Q0002+051 G1 and Q0836+113 G1, we have determined that large-scale galactic outflows might be giving rise to the observed $\mathrm{Mg}$ II absorption kinematics. Both galaxies have a SFR $\sim 6.5 M_{\odot} \mathrm{yr}^{-1}$ and $\Sigma \geqslant 0.35$ $M_{\odot} \mathrm{yr}^{-1} \mathrm{kpc}^{-2}$ and $\Sigma \geqslant 0.14 M_{\odot} \mathrm{yr}^{-1} \mathrm{kpc}^{-2}$, respectively. These SFRs and $\Sigma$ s are typically found for galaxies exhibiting outflow velocities of several hundred $\mathrm{km} \mathrm{s}^{-1}$. The Mg II absorption velocities associated with the two galaxies span both sides of their systemic velocity. Such profiles have been interpreted, in both our simulations and at high redshift, as signatures of outflows.

3. We find that the observed Mg II absorption velocity spread and optical depth distribution may be a function of galaxy inclination. Galaxies with higher inclination exhibit a Mg II absorption velocity spread of $\sim 300 \mathrm{~km} \mathrm{~s}^{-1}$ with a somewhat even distribution of optical depths, whereas, galaxies with lower inclinations exhibit a narrower velocity spread of $\sim 100 \mathrm{~km} \mathrm{~s}^{-1}$ with a clear optical depth peak at $v \sim 100 \mathrm{~km} \mathrm{~s}^{-1}$. A K-S test shows that the Mg II optical depth distributions for the high and low inclination bins are not consistent at the $3.3 \sigma$ level. These results suggest that the absorbing gas is either disk like or the spatial distribution and kinematics of the structures producing the absorption (i.e., filaments, tidal streams, satellites etc.) are closely coupled to the disk orientation.

4. We employed simple rotating disk halo models to examine whether disk-like rotation is consistent with the observed galaxy-gas kinematics. For model parameters that allow for a $1 \mathrm{Mpc}$ gas scale height and maximum rotation velocity (rigid rotation) the bulk of the observed absorption kinematics can be explained by co-rotation with the galaxy. In all cases, the rotating disk halo models we present are unable reproduce the full spread of observed $\mathrm{Mg}_{\text {II }}$ absorption velocities. This model is a highly unrealistic representation of galaxy gas. When the parameters are relaxed to better reflect reasonable gas scale heights and a slowing of the rotation speed with height above the disk plane, the relative proportion of the gas velocity spread that can be made consistent with galaxy co-rotation diminishes such that some absorbers cannot have but a tiny fraction explained by co-rotation. In this simple scenario, even if some of the absorbing gas arises in a thick disk, what we learn from the exercise is that some additional type of dynamical process (such as infall, outflow, supernovae winds, etc.) must be invoked to explain the range of absorption velocities that cannot be made consistent with the simple rotating disk halo model.

5. In two quasar fields, we find pairs of galaxies that align in velocity within $\sim 100 \mathrm{~km} \mathrm{~s}^{-1}$ of a single, saturated Mg II absorption system. For one case, the observed velocity range of the strong saturated component can be explained by a rotating disk model only if both galaxies contribute to the absorption. This challenges the idea that an individual Mg II absorber can be assigned to a single galaxy, and understood as an isolated halo.

6. In the simulations, $\mathrm{Mg}$ II absorption selects gas structures such as metal enriched tidal streams, filaments, small satellite galaxies, and the region within $\sim 20 \mathrm{kpc}$ of the galaxy. Together, these structures extend roughly $\sim 100 \mathrm{kpc}$ around the galaxy, suggesting that galaxy "halos" are a complex composite of the these various structures.

7. For this simulated galaxy the $\mathrm{Mg}$ II covering fraction is $\sim 10 \%$, which is below the current observational estimated means of 20\%-80\% (Tripp \& Bowen 2005; Kacprzak et al. 2008; Barton \& Cooke 2009). This may reflect a need for additional tuning of the feedback/baryon physics in the simulations, or indicates current observational biases.

8. In the simulations, DLA H I column densities arise in lowmass satellite galaxies at impact parameters as large as $\sim 100 \mathrm{kpc}$. These galaxies are below the detection limits of deep HST images. Although, the covering fractions of these dense regions are low, this might explain why some bright galaxies at DLA redshifts are found at large impact parameters.

9. In the simulations, the majority of the $\mathrm{Mg}$ II absorbing gas is infalling in filaments and tidal streams toward the galaxy with velocities between $-200 \mathrm{~km} \mathrm{~s}^{-1} \leqslant v_{r} \leqslant$ $-180 \mathrm{~km} \mathrm{~s}^{-1}$. The velocity offset probability distribution (relative to the simulated galaxy) spans $\sim 200 \mathrm{~km} \mathrm{~s}^{-1}$ with lowest probability of detecting Mg II at the galaxy systematic velocity. Thus, observed $\mathrm{Mg}$ II absorption velocities can fall within the range of the galaxy rotation curve velocities, even though the gas arises in a variety of kinematics structures.

The gas structures selected by $\mathrm{Mg}$ II in the simulations (see Figures 9-12) cannot be described as simple thick disks or 
spherical halos. If the simulations reflect reality, it would appear that $\mathrm{Mg}$ II absorption arises in large $\sim 100 \mathrm{kpc}$ halos that are built from the local cosmological environment of a moderate mass galaxy. Complicating the picture is the fact that we find groups and pairs of galaxies that align in velocity within $\sim 100 \mathrm{~km} \mathrm{~s}^{-1}$ of a single $\mathrm{Mg}$ II absorption system. This challenges the idea that an individual $\mathrm{Mg}$ II absorber can be assigned to a single galaxy or understood as an isolated halo. Though considered subcomponents of halos, smaller scale structures like the Magellanic-type galaxies and tidal streams, may contribute significantly to the detections of $\mathrm{Mg}$ II absorption (York et al. 1986; Kacprzak et al. 2007). These considerations lead us to suggest that galaxies and $\mathrm{Mg}$ II absorbers should be studied and modeled in a environmental context if they are to be fully understood.

In the simulations, the kinematics are closely coupled to the gas structures (i.e., filaments, tidal streams, small satellite galaxies, and the inner $20 \mathrm{kpc}$ of the central galaxy). As observed in our data, the simulated $\mathrm{Mg}$ II absorption velocities fall within the range of the galaxy rotation velocities, and rarely at the galaxy systematic velocity. Thus, the simulations suggest that observing $\mathrm{Mg}$ II absorption velocities consistent with the galaxy rotation curves can naturally occur even if the absorption arises in many different structures in the complex environment of the galaxy. It is these structures that comprise halos.

A natural extension of the work presented here would be to perform a similar study (simulations and observations) that incorporates the kinematics of higher ionization $\mathrm{C}$ IV $\lambda \lambda 1548,1550$ and $O$ VI $\lambda \lambda 1031,1037$ doublet absorption. These ions probe lower density and/or higher temperature structures and provide a more comprehensive view of the gaseous environment around galaxies. Future observations with the Cosmic Origins Spectrograph are perfectly suited for the galaxy sample presented in this paper. It is also important to expand the number of galaxy environments studied in the simulations.

We thank Greg Wirth for his help and advice with ESI/Keck. We are grateful to A. Kravtsov for providing the hydro code. We are in debt to N. Gnedin creating the graphics package IFRIT. We thank Aneta Siemiginowska for her discussion regarding the X-ray data of Q127-145. We express our gratitude to the anonymous referee for a careful reading and for insightful comments that lead to an improved manuscript. C.W.C and G.G.K were funded by the NSF grant AST 0708210. G.G.K was partially funded by the NMSU Graduate Research Enhancement Grant. M.T.M thanks the Australian Research Council for a QEII Research Fellowship (DP0877998). Most of the data presented herein were obtained at the W. M. Keck Observatory, which is operated as a scientific partnership among the California Institute of Technology, the University of California and the National Aeronautics and Space Administration. The Observatory was made possible by the generous financial support of the W. M. Keck Foundation. Some observations were made with the NASA/ESA Hubble Space Telescope, obtained from the Data Archive at the Space Telescope Science Institute, which is operated by the Association of Universities for Research in Astronomy, Inc., under NASA contract NAS 5-26555. Some of this research was based on observations made with ESO Telescopes at the Paranal Observatories under program IDs listed in Table 1. The computer simulations presented in this paper were performed at the National Energy Research Scientific Computing Center (NERSC) of the Lawrence Berkeley National Laboratory.
Facilities: HST (WFPC-2), Keck II (ESI), Keck I (HIRES), VLT (UVES)

\section{REFERENCES}

Bahcall, J. N., Kirhakos, S., Saxe, D. H., \& Schneider, D. P. 1997, ApJ, 479 642

Barton, E. J., \& Cooke, J. 2009, AJ, 138, 1817

Bechtold, J., Green, R. F., Weymann, R. J., Schmidt, M., Estabrook, F. B., Sherman, R. D., Wahlquist, H. D., \& Heckman, T. M. 1984, ApJ, 281, 76

Bergeron, J., \& Boissé, P. 1991, A\&A, 243, 334

Bergeron, J., Cristiani, S., \& Shaver, P. A. 1992, A\&A, 257, 417

Bergeron, J., \& Kunth, D. 1984, MNRAS, 207, 263

Bond, N. A., Churchill, C. W., Charlton, J. C., \& Vogt, S. S. 2001, ApJ, 557, 761

Bouché, N., Murphy, M. T., Péroux, C., Davies, R., Eisenhauer, F., Förster Schreiber, N. M., \& Tacconi, L. 2007, ApJ, 669, L5

Bowen, D. V., Blades, J. C., \& Pettini, M. 1995, ApJ, 448, 634

Bowen, D. V., Pettini, M., \& Blades, J. C. 2002, ApJ, 580, 169

Burkert, A., \& Lin, D. N. C. 2000, ApJ, 537, 270

Cabanac, R. A., Valls-Gabaud, D., \& Lidman, C. 2008, MNRAS, 386, 2065

Charlton, J. C., \& Churchill, C. W. 1996, ApJ, 465, 631

Ceverino, D., \& Klypin, A. 2009, ApJ, 695, 292

Charlton, J. C., \& Churchill, C. W. 1998, ApJ, 499, 181

Chen, H.-W., Lanzetta, K. M., Webb, J. K., \& Barcons, X. 1998, ApJ, 498, 77

Chen, H.-W., \& Tinker, J. L. 2008, ApJ, 687, 745

Chun, M. R., Gharanfoli, S., Kulkarni, V. P., \& Takamiya, M. 2006, AJ, 131, 686

Chung, A., van Gorkom, J. H., Kenney, J. D. P., \& Vollmer, B. 2007, ApJ, 659, L115

Churchill, C. W. 1997, PhD thesis, Univ. California, Santa Cruz

Churchill, C. W., \& Charlton, J. C. 1999, AJ, 118, 59

Churchill, C. W., Kacprzak, G. G., \& Steidel, C. C. 2005, in IAU Coll. 199, Probing Galaxies through Quasar Absorption Lines, ed. P. R. Williams, C.-G Shu, \& B. Ménard (Cambridge: Cambridge Univ. Press), 24

Churchill, C. W., Mellon, R. R., Charlton, J. C., Jannuzi, B. T., Kirhakos, S., Steidel, C. C., \& Schneider, D. P. 2000, ApJS, 130, 91

Churchill, C. W., Rigby, J. R., Charlton, J. C., \& Vogt, S. S. 1999, ApJS, 120, 51

Churchill, C. W., Steidel, C. C., \& Vogt, S. S. 1996, ApJ, 471, 164

Churchill, C. W., \& Vogt, S. S. 2001, AJ, 122, 679

Côté, S., Wyse, R. F. G., Carignan, C., Freeman, K. C., \& Broadhurst, T. 2005, ApJ, 618, 178

Dekker, H., D’Odorico, S., Kaufer, A., Delabre, B., \& Kotzlowski, H. 2000, Proc. SPIE, 4008, 534

Ellison, S. L., Mallén-Ornelas, G., \& Sawicki, M. 2003, ApJ, 589, 709

Erb, D. K., Steidel, C. C., Shapley, A. E., Pettini, M., Reddy, N. A., \& Adelberger, K. L. 2006, ApJ, 647, 128

Ferland, G. J., Korista, K. T., Verner, D. A., Ferguson, J. W., Kingdon, J. B., \& Verner, E. M. 1998, PASP, 110, 761

Fraternali, F., Oosterloo, T., Sancisi, R., \& van Moorsel, G. 2001, ApJ, 562, L47

Guillemin, P., \& Bergeron, J. 1997, A\&A, 328, 499

Haardt, F., \& Madau, P. 1996, ApJ, 461, 20

Heald, G. H., Rand, R. J., Benjamin, R. A., \& Bershady, M. A. 2007, ApJ, 663, 933

Heckman, T. M. 2002, in ASP Conf. Ser. 254, Extragalactic Gas at Low Redshift, ed. J. S. Mulchaey \& J. Stocke (San Francisco, CA: ASP), 292

Heckman, T. M. 2003, RevMexAA Conf. Ser., 17, 47

Kacprzak, G. G., Churchill, C. W., Steidel, C. C., \& Murphy, M. T. 2008, AJ, 135,922

Kacprzak, G. G., Churchill, C. W., Steidel, C. C., Murphy, M. T., \& Evans, J. L. 2007, ApJ, 662, 909

Kaufmann, T., Bullock, J. S., Maller, A. H., Fang, T., \& Wadsley, J. 2009, MNRAS, 396, 191

Kennicutt, R. C., Jr. 1998, ApJ, 498, 541

Kewley, L. J., Geller, M. J., \& Jansen, R. A. 2004, AJ, 127, 2002

Klypin, A., Kravtsov, A. V., Bullock, J. S., \& Primack, J. R. 2001, ApJ, 554, 903

Kravtsov, A. V. 1999, Ph.D. thesis, New Mexico State Univ.

Kravtsov, A. V. 2003, ApJ, 590, L1

Lane, W., Smette, A., Briggs, F., Rao, S., Turnshek, D., \& Meylan, G. 1998, AJ, 116,26

Lanzetta, K. M., \& Bowen, D. V. 1992, ApJ, 391, 48L

Law, D. R., Steidel, C. C., Erb, D. K., Larkin, J. E., Pettini, M., Shapley, A. E., \& Wright, S. A. 2007, ApJ, 669, 929

Le Brun, V., Bergeron, J., Boisse, P., \& Christian, C. 1993, A\&A, 279, 33 
Lin, D. N. C., \& Murray, S. D. 2000, ApJ, 540, 170

Lopez, S., et al. 2008, ApJ, 679, 1144

Lowenthal, J. D., Hogan, C. J., Green, R. F., Woodgate, B., Caulet, A., Brown, L., \& Bechtold, J. 1995, ApJ, 451, 484

Maller, A. H., \& Bullock, J. S. 2004, MNRAS, 355, 694

Mo, H. J., \& Miralda-Escude, J. 1996, ApJ, 469, 589

Monet, D., et al. 1998, USNO-SA2.0: A Catalog of Astrometric Standards (Washington, DC: US Nav. Obs.)

Murphy, M. T. 2006, UVES POPLER, http://astronomy.swin.edu.au/ mmurphy/ UVES popler.htm

Navarro, J. F., \& Steinmetz, M. 2000, ApJ, 538, 477

Nestor, D. B., Rao, S. M., Turnshek, D. A., Monier, E., Lane, W. M., \& Bergeron, J. 2002, in ASP Conf. Ser. 254, Extragalactic Gas at Low Redshift, ed. J. S. Mulchaey \& J. Stocke (San Francisco, CA: ASP), 34

Oosterloo, T., Fraternali, F., \& Sancisi, R. 2007, AJ, 134, 1019

Pettini, M., Shapley, A. E., Steidel, C. C., Cuby, J.-G., Dickinson, M., Moorwood, A. F. M., Adelberger, K. L., \& Giavalisco, M. 2001, ApJ, 554, 981

Prochaska, J. X., \& Wolfe, A. M. 1997, ApJ, 474, 140

Prochaska, J. X., Wolfe, A. M., Howk, J. C., Gawiser, E., Burles, S. M., \& Cooke, J. 2007, ApJS, 171, 29

Rand, R. J. 2000, ApJ, 537, L13

Rao, S. M., Nestor, D. B., Turnshek, D. A., Lane, W. M., Monier, E. M., \& Bergeron, J. 2003, ApJ, 595, 94

Rao, S. M., \& Turnshek, D. A. 2000, ApJS, 130, 1

Rigby, J. R., Charlton, J. C., \& Churchill, C. W. 2002, ApJ, 565, 743

Rubin, K. H. R., Prochaska, J. X., Koo, D. C., Phillips, A. C., \& Weiner, B. J. 2009, arXiv:0907.0231

Sancisi, R., Fraternali, F., Oosterloo, T., \& van der Hulst, T. 2008, A\&AR, 15 , 189

Sancisi, R., Fraternali, F., Oosterloo, T., \& van Moorsel, G. 2001, in ASP Conf. Ser. 240, Gas and Galaxy Evolution, ed. J. E. Hibbard, M. Rupen, \& J. H. van Gorkom (San Francisco, CA: ASP), 241

Sargent, W. L. W., Boksenberg, A., \& Steidel, C. C. 1988, ApJS, 68, 539

Shapley, A. E., Steidel, C. C., Pettini, M., \& Adelberger, K. L. 2003, ApJ, 588, 65
Sheinis, A. I., Bolte, M., Epps, H. W., Kibrick, R. I., Miller, J. S., Radovan, M. V., Bigelow, B. C., \& Sutin, B. M. 2002, PASP, 114, 851

Siemiginowska, A., Bechtold, J., Aldcroft, T. L., Elvis, M., Harris, D. E., \& Dobrzycki, A. 2002, ApJ, 570, 543

Siemiginowska, A., Stawarz, Ł., Cheung, C. C., Harris, D. E., Sikora, M., Aldcroft, T. L., \& Bechtold, J. 2007, ApJ, 657, 145

Simard, L., et al. 2002, ApJS, 142, 1

Simcoe, R. A., Sargent, W. L. W., Rauch, M., \& Becker, G. 2006, ApJ, 637, 648

Steidel, C. C., Adelberger, K. L., Shapley, A. E., Pettini, M., Dickinson, M., \& Giavalisco, M. 2003, ApJ, 592, 728

Steidel, C. C., Dickinson, M., Meyer, D. M., Adelberger, K. L., \& Sembach, K. R. 1997, ApJ, 480, 586

Steidel, C. C., Dickinson, M., \& Persson, S. E. 1994, ApJ, 437, L75

Steidel, C. C., Kollmeier, J. A., Shapely, A. E., Churchill, C. W., Dickinson, M., \& Pettini, M. 2002, ApJ, 570, 526

Steidel, C. C., \& Sargent, W. L. W. 1992, ApJS, 80, 1

Swaters, R. A., Sancisi, R., \& van der Hulst, J. M. 1997, ApJ, 491, 140

Tasker, E. J., \& Bryan, G. L. 2006, ApJ, 641, 878

Tinker, J. L., \& Chen, H.-W. 2008, ApJ, 679, 1218

Tremonti, C. A., Moustakas, J., \& Diamond-Stanic, A. M. 2007, ApJ, 663 , L77

Tripp, T. M., \& Bowen, D. V. 2005, in IAU Coll.199, Probing Galaxies through Quasar Absorption Lines, ed. P. R. Williams, C.-G. Shu, \& B. Ménard (Cambridge: Cambridge Univ. Press), 5

Turnshek, D. A., Wolfe, A. M., Lanzetta, K. M., Briggs, F. H., Cohen, R. D., Foltz, C. B., Smith, H. E., \& Wilkes, B. J. 1989, ApJ, 344, 567

Tytler, D., Boksenberg, A., Sargent, W. L. W., Young, P., \& Kunth, D. 1987, ApJS, 64, 667

Vogt, N. P., Forbes, D. A., Phillips, A. C., Gronwall, C., Faber, S. M., Illingworth, G. D., \& Koo, D. C. 1996, ApJ, 465, L15

Vogt, S. S., et al. 1994, Proc. SPIE, 2198, 362

Weiner, B. J., et al. 2009, ApJ, 692, 187

White, S. D. M., \& Frenk, C. S. 1991, ApJ, 379, 52

York, D. G., Dopita, M., Green, R., \& Bechtold, J. 1986, ApJ, 311, 610

Zibetti, S., Ménard, B., Nestor, D. B., Quider, A. M., Rao, S. M., \& Turnshek, D. A. $2007, \mathrm{ApJ}, 658,161$ 